\title{
Super-immunity by broadly protective nanobodies to sarbecoviruses
}

Yufei Xiang ${ }^{1 *}$, Wei Huang ${ }^{2 *}$, Hejun Liu ${ }^{3 *}$, Zhe Sang $^{4 *}$, Sham Nambulli5,6, Jérôme Tubiana ${ }^{7,8}$, Kevin L Williams $J^{5}{ }^{5,6}$, W Paul Duprex ${ }^{5,6}$, Dina Schneidman-Duhovny ${ }^{7}$, Ian A. Wilson ${ }^{3,9}$, Derek J. Taylor ${ }^{2,10}$, and Yi Shi ${ }^{1,4, \S}$

${ }^{1}$ Department of Cell Biology, University of Pittsburgh, Pittsburgh, PA, 15213, USA

${ }^{2}$ Department of Pharmacology, Case Western Reserve University, Cleveland, OH 44106, USA

${ }^{3}$ Department of Integrative Structural and Computational Biology, The Scripps Research Institute, La Jolla, CA, 92037, USA

${ }^{4}$ The University of Pittsburgh and Carnegie Mellon University Program for Computational Biology, Pittsburgh, PA, 15213, USA

${ }^{5}$ Center for Vaccine Research, University of Pittsburgh, Pittsburgh, PA, 15213, USA

${ }^{6}$ Department of Microbiology and Molecular Genetics, University of Pittsburgh, Pittsburgh, PA, 15213, USA

${ }^{7}$ School of Computer Science and Engineering, The Hebrew University of Jerusalem, Israel

${ }^{8}$ Blavatnik School of Computer Science, Tel Aviv University, Israel

${ }^{9}$ Skaggs Institute for Chemical Biology, The Scripps Research Institute, La Jolla, CA, 92037, USA

${ }^{10}$ Department of Biochemistry, Case Western Reserve University, Cleveland, OH 44106, USA

*These authors contributed equally to this work

§Correspondence: yi.shi@pitt.edu

\section{Abstract}


Vaccine boosters and infection can facilitate the development of SARS-CoV-2 antibodies with improved potency and breadth. Here, we observed super-immunity in a camelid extensively immunized with the SARSCoV-2 receptor-binding domain (RBD). We rapidly isolated a large repertoire of specific ultrahigh-affinity nanobodies that bind strongly to all known sarbecovirus clades using integrative proteomics. These pansarbecovirus nanobodies (psNbs) are highly effective against SARS-CoV and SARS-CoV-2 variants including the Omicron, with the best median neutralization potency at single-digit $\mathrm{ng} / \mathrm{ml}$. Structural determinations of 13 psNbs with the SARS-CoV-2 spike or RBD revealed five epitope classes, providing insights into the mechanisms and evolution of their broad activities. The highly evolved psNbs target small, flat, and flexible epitopes that contain over $75 \%$ of conserved RBD surface residues. Their potencies are strongly and negatively correlated with the distance of the epitopes to the receptor binding sites. A highly potent, inhalable and bispecific psNb (PiN-31) was developed. Our findings inform on the development of broadly protective vaccines and therapeutics.

One sentence summary: Successive immunization of SARS-CoV-2 RBD in a camelid enhanced the development of super-immunity and isolation and systematic characterization of a large repertoire of ultrahighaffinity pan-sarbecovirus single-chain $\mathrm{V}_{\mathrm{H}} \mathrm{H}$ antibodies to understand the evolution of this potent and broad immune response. 


\section{MAIN}

SARS-related coronaviruses (sarbecoviruses) including SARS-CoV and SARS-CoV-2, are among the most pressing threats to global health. The high genetic diversity, frequent recombination, large natural reservoirs, and proximity to heavily populated areas across continents, underlie the recurrent zoonotic risks of sarbecoviruses and other circulating coronaviruses $(1,2)$. Thus, there is an urgent need to develop broad, effective, and complementary interventions against the currently evolving pandemic as well as future threats. Emerging evidence indicates that $B$ cells isolated from convalescent and infected-then-vaccinated individuals continue to evolve, producing antibodies with increased potency against SARS-CoV-2 antigenic drift (3-7). Most antibodies target the spike RBD, which dominates immunogenicity and neutralizing activities of convalescent and vaccinated sera (8). Only a small number of Immunoglobulin G (lgG) antibodies against the large sarbecovirus family have been successfully isolated with varied breadth and potency (9-13). Moreover, an understanding of the full dynamic repertoire of broadly neutralizing antibodies and mechanisms that shape SARS-CoV-2 super-immunity is still lacking (3).

By camelid immunization and proteomics, we recently identified thousands of high-affinity RBD nanobodies (Nbs) that potently neutralize SARS-CoV-2 (14). Most ultrapotent Nbs bind the highly variable human ACE2 (hACE2) receptor-binding sites (RBS) and are therefore less effective against evolving variants.

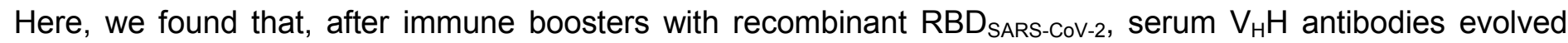
with substantially improved activities not only against the variants of concern (VOCs) but also to a broad spectrum of sarbecoviruses. To understand the broad serologic activities, we isolated and systematically characterized 100 high-affinity pan-sarbecovirus nanobodies (psNbs) with superior potency and breadth and developed an ultrapotent, bispecific and aerosolizable psNb (PiN-31). Structural determinations of 13 diverse psNbs with the spike or RBD by cryoEM and X-ray crystallography revealed five classes with marked diversity within dominant classes. Our analysis offers insights into the remarkable evolution of serologic responses towards broad activity against sarbecoviruses.

\section{Identification and characterization of a large repertoire of potent pan-sarbecovirus $\mathrm{Nbs}$}

A llama was initially immunized with SARS-CoV-2 RBD-Fc fusion protein and the initial bleed was collected approximately 2 months after priming and three boosts (14). We then re-immunized with four additional boosts for 2 months before the booster bleed was collected (see Methods in Supplementary Materials). The polyclonal $\mathrm{V}_{\mathrm{H}} \mathrm{H}$ mixture of the booster bleed showed higher affinity to RBD $\mathrm{SARS}-\mathrm{CoV}-2_{2}$ (ELISA IC 50 S of $43 \mathrm{pM}$ ) compared to that of the initial bleed $\left(\mathrm{IC}_{50}\right.$ of $\left.130 \mathrm{pM}\right)$ (Fig. 1B, fig. S1A). The $\mathrm{V}_{\mathrm{H}} \mathrm{H}$ mixture also maintained excellent neutralization potency $\left(\mathrm{IC}_{50} \mathrm{~s}\right.$ between 0.3-0.6 nM) against the Wuhan-Hu-1 strain as well as Alpha and Lambda VOCs (fig. S1B. Notably, compared to the initial $\mathrm{V}_{\mathrm{H}} \mathrm{Hs}$, the neutralization potencies after booster were substantially increased to Beta, Delta and SARS-CoV $\left(\mathrm{IC}_{50} \mathrm{~S}\right.$ of $0.58,1.90$ and $1.65 \mathrm{nM}$, respectively) corresponding to $6.0,2.3$, and 9.3 fold improvements (fig. S1B). Surprisingly, the booster was also associated 
with strong and broad activities against the full spectrum of sarbecoviruses (Fig. 1, A and B). The relative

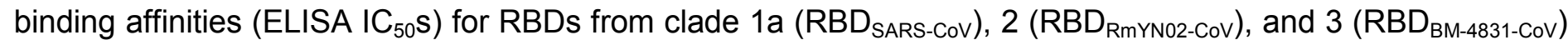
were $0.08,0.09$, and $0.14 \mathrm{nM}$, respectively, which correspond to $8.5,7.2$, and 8.6 fold improvements over the initial bleed (fig. S1A).

Next, we employed quantitative $\mathrm{Nb}$ proteomics (15) to identify high-affinity psNbs that confer broadspectrum activity in immunized sera (see Methods). This technology rapidly identified hundreds of distinct CDR3 families, from which a fraction of highly divergent Nbs were expressed and characterized. A total of 100 $\mathrm{Nbs}$ that interacted strongly with $\mathrm{RBD}_{\mathrm{SARS}-\mathrm{CoV}-2}($ Clade $1 \mathrm{~b}$ ) were confirmed to cross-react with other sarbecovirus clades (Fig. 1C, table S1). Of these, 23\%, 35\%, and 42\% were found to bind two, three, and all four sarbecovirus clades, respectively. Consistent with total polyclonal $\mathrm{V}_{\mathrm{H}} \mathrm{H}$ activity, psNbs isolated from the booster show broader pan-sarbecovirus activity than the initial bleed (fig. S2B). A substantial fraction (42\%) was able to potently neutralize SARS-CoV-2 below $500 \mathrm{nM}$, with the best $\mathrm{IC}_{50}$ of $1.2 \mathrm{ng} / \mathrm{ml}(77 \mathrm{pM}$, 2-67), and a median of $0.25 \mu \mathrm{g} / \mathrm{ml}(17 \mathrm{nM})$ (table S1). Network analysis revealed that the psNbs are dominated by multiple clusters that span a large spectrum of physicochemical properties including isoelectric point ( $\mathrm{pl}$ ) and hydropathy (Fig. 1D, fig. S2A). The three largest clusters are each composed of potent neutralizers (with the best $\mathrm{IC}_{50}$ of $6.8 \mathrm{ng} / \mathrm{ml}$ ) that bind strongly to at least three sarbecovirus clades (table S1). A smaller cluster (represented by 2-62 and 2-63) showed broad activity, yet only weakly neutralized SARS-CoV-2 in vitro (IC 50 S between $40-132 \mu \mathrm{g} / \mathrm{ml}$, table S1, fig. S6). Other small clusters represented by 2-19, 2-16, 2-3 and 2-24 did not show neutralization at up to $37.5 \mu \mathrm{g} / \mathrm{ml}$.

The psNbs were further classified by epitope binning using size exclusion chromatography (SEC). psNb-RBD ${ }_{\text {SARS-CoV-2 }}$ complexes were competed with high-affinity benchmark Nbs (Nb21, Nb105, and Nb36) that bind distinct and well-characterized epitopes (16) (fig. S3, A and B, Methods). psNbs fall into five groups: group A (3\%) competes with Nb21 and targets RBS, group B (33\%) with Nb105, group C (9\%) with Nb36, and group D (17\%) does not compete with any benchmark Nbs. Approximately one third of psNbs (Group E, 38\%) bind strongly to RBDs based on ELISA, but do not neutralize pseudotyped SARS-CoV-2 efficiently. We selected a high-affinity psNb (2-47) from group $E$ and confirmed its strong binding to the fully glycosylated but not the de-glycosylated RBD (fig. S3C, Methods). It is possible that 2-47 can target an epitope encompassing the conserved, glycosylated RBD residue(s) (e.g., N343) (17). Together, these experiments suggest that a large cohort of high-affinity and divergent psNbs targeting multiple dominant RBD epitopes contribute to the broad-spectrum serologic activities.

17 psNbs spanning four SEC groups (A-D) were tested for binding to RBDs of five SARS-CoV-2 variants, including the fast-spreading Omicron, and other 18 different sarbecovirus RBDs. These psNbs bind strongly to all variants that were evaluated. 16 of the 17 psNbs bind to all four clades (Fig. 2A). Interestingly, seven psNbs (such as 2-31 and 2-45) have exceptionally broad activities and bind strongly to all 24 RBDs with a median ELISA IC 50 of $3 \mathrm{nM}$ (Fig. 2A, fig. S4, table S1). Two representative psNbs were measured for 


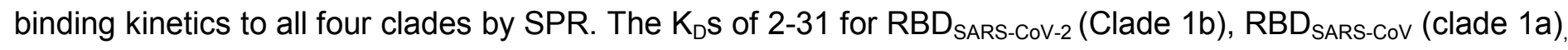
$\mathrm{RBD}_{\mathrm{RmYN02-Cov}}\left(\right.$ clade 2), $\mathrm{RBD}_{\mathrm{BM}-4831-\operatorname{Cov}}$ (clade 3) are $<1 \mathrm{pM}, 3.96 \mathrm{pM}, 0.59 \mathrm{nM}$ and $3.60 \mathrm{nM}$, respectively(Fig. 2D, fig. S7, A and B). The binding of 2-45 is equally strong for clades $1 \mathrm{~b}, 2$ and $3(0.39 \mathrm{nM}, 1.4 \mathrm{nM}$ and 44 pM,respectively) and more moderate for clade 1a (304 nM) (Fig. 2E, fig. S7, C and D). Of note, these psNbs are highly specific to sarbecoviruses and do not cross-react with a human whole protein extract at high concentrations (up to $8 \mu \mathrm{M}$ ) (Fig. 2A).

All but one of these 17 psNbs can potently inhibit SARS-CoV-2 and VOCs in vitro, based on a pseudovirus assay, and plaque reduction neutralization test (PRNT) against the Munich strain and Delta VOC (Fig. 2B and C, figs. S5 and S6, Methods). Group A Nb 2-67, which preferentially binds to clade $1 \mathrm{~b}$, is extremely potent for SARS-CoV-2 and VOCs with a median neutralization potency of $1.2 \mathrm{ng} / \mathrm{ml}$. Group C (1-22, 2-10 and 2-45) neutralizes VOCs at single-digit $\mu \mathrm{g} / \mathrm{ml}$, but is less effective against SARS-CoV. 2-63 (group D) has exceptional breath while the other group D psNb 2-28 has limited breadth. Both weakly neutralize SARSCoV-2 ( $\left.\mathrm{IC}_{50} 7-132 \mu \mathrm{g} / \mathrm{ml}\right)$. The remaining psNbs belong to group $\mathrm{B}$ and show comparably high potency against SARS-CoV. The median potency for SARS-CoV-2 and VOCs is $86 \mathrm{ng} / \mathrm{ml}$ with the most potent (2-55) at 6 $\mathrm{ng} / \mathrm{ml}$. Notably, psNbs are usually highly stable and can withstand aerosolization without compromising activity as exemplified by 2-31 and 2-45 (Fig. 2F, fig. S7, E and F, Methods). To demonstrate the high bioengineering potentials of psNbs, we developed a bispecific construct ( $\mathrm{PiN}-31)$ by fusing these two potent and broadspectrum psNbs, which cover two distinct epitopes based on SEC. Compared to the monomers, the potency of PiN-31 improves by an order of magnitude to $0.4 \mathrm{nM}$ based on the PRNT assay (Fig. 2G).

\section{Diversity, convergence and evolution of broadly neutralizing psNbs}

To understand the mechanisms of broad neutralization, we implemented cryoEM to interrogate the structures of 11 psNbs in complex with the SARS-CoV-2 spike or RBD (table S2). Additionally, two atomic psNb: RBD structures were determined by X-ray crystallography (table S3). Epitope clustering based on high-resolution structures revealed five primary and distinct epitope classes in $\mathrm{RBD}_{\mathrm{SARS}-\mathrm{CoV}-2}$ (Fig. 3A). None of our psNbs (except for 2-57) overlap with key RBD mutations in variants including Alpha, Beta, Delta, Lambda, Gamma, and Omicron (Fig. 3B), in contrast to most other Nbs that have been structurally characterized (18-25)(fig. S8). Over three-quarters of the solvent-exposed and highly conserved RBD residues (sequence identity $>85 \%$ ) are covered by psNbs (see Methods). Superposition onto the spike structure reveals that psNbs preferentially lock RBDs in the 3-up conformation (Fig. 3C). Despite having different epitopes and orientations, the small size of Nbs facilitates simultaneous binding of three copies to the spike trimer in distinct and highly symmetric configurations.

The Class II psNbs are overrepresented in our collection. Phylogenetic analysis reveals that while psNbs are diverse, those isolated from the booster converge more than the initial bleed (Fig. 4A). Two subclasses within the class II (A and B) differ in epitopes and Nb binding orientation (Fig. 4, B and D). Nevertheless, II(A) and II(B) psNbs share a conserved hydrophobic core epitope (aa 377-386) (Fig. 4C) 
containing two bulky hydrophobic residues (F377 and Y380), and this region is stabilized by a disulfide bond

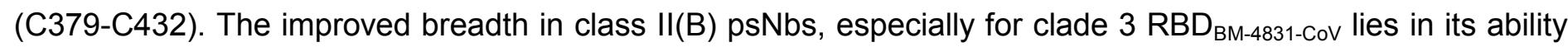
to target an additional conserved region with peripheral charged residues (aa 412-415, 427-429). In contrast, subclass II(A) psNbs bind to a less conserved epitope that is composed of primarily hydrophobic residues (e.g., L368, A372, and Y508) (Fig. 4C). Notably, A372 on SARS-CoV-2 (clade 1a) is substituted to S/T in other clades, forming a consensus glycosylation motif $\left({ }^{370} \mathrm{NST} / \mathrm{S}^{372}\right)(26)$, which may explain the reduced neutralization potency of class II (A) psNbs versus class (B) psNbs against SARS-CoV (Fig. 2B, 4B).

Class II(B) psNbs comprise two large clusters possessing the best breadth and potency (Fig. 1D). While the epitopes largely overlap in the complex structure, the molecular interactions vary substantially. For example, two related psNbs (2-38 and 1-21) are characterized by a short CDR3 (13 aa) forming a $\beta$-strand hairpin conformation. Yet, psNb 2-38 is an all-clade binder whereas 1-21 binds strongly to all but $R_{B D} D_{B M-4831-C o v}$ (clade 3). The difference in binding is likely due to the $\mathrm{K} 378 \mathrm{Q}$ substitution in the RBD, which disrupts a critical

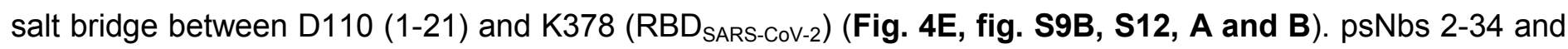
2-31 (from the other dominant cluster) bind all 24 RBDs strongly with potent antiviral activities. The interactions are predominantly mediated by a long CDR3 loop (18 aa) with a small helix. These Nbs form hydrophobic interactions and also bind strongly to conserved charged residues (such as D427 and R408) through electrostatic interactions (Fig. 4F, fig. S9A, S12, C and D).

Class II(A) psNb (2-57) shares high sequence similarity with Nb105 (isolated from the initial bleed). The major difference lies in their CDR3 heads. Here, a small CDR3 loop of ${ }^{109}{ }^{D L F}{ }^{111}$ in Nb105 is substituted by ${ }^{109} \mathrm{QST}^{111}$ in 2-57, which inserts into two conserved pockets (RBD residues F377,Y369,F374 and residues S375,T376,Y508,V407) forming extensive hydrophobic interactions with the RBD (Fig. 4G, fig. S10, S11, S12E). This substitution allows psNb 2-57 to tolerate sequence variation better, exhibiting over 100 fold higher affinities for clade 2 RBDs (table S1).

Class I psNbs that bind the RBS are extremely potent yet rare $(\sim 3 \%)$. The structure of the ultrapotent 2-67 was resolved by cryoEM in complex with the RBD (fig. S13). Superposition of 2-67:RBD and hACE2:RBD (PDB 6M0J) (27) structures revealed major clashes between all three $\mathrm{Nb}$ framework regions and two CDRs (2 and 3) with hACE2 (aa 26-37, 66-87, 91-109, 187-194) (Fig. 5A). 2-67 targets the protruding RBD ridge (aa 472-490) (Fig. 5B, fig. S15A). Binding energy analysis (Methods) reveals that compared to other RBS Nbs (18-25), the RBD binding epitope of 2-67 is minimally contributed by critical VOC residues (Fig. 5C, table S5). Here, 2-67 utilizes all CDRs to form extensive networks of hydrophobic and nonpolar interactions with two critical RBD residues that are not present as VOC mutations (F486 and Y489), and therefore, can achieve ultrahigh affinity for SARS-CoV-2 and retain strong binding and neutralization against the variants (Fig. 5D). Moreover, 2-67 can outcompete ultrahigh-affinity Nb21 for RBS binding, thereby further corroborating the ultrahigh affinity of this interaction (fig. S14A) (14). Interestingly, quantitative mass spectrometric analysis reveals 
that ultrapotent RBS binders (e.g., Nbs 20 and 21) identified from the initial bleed were absent in the booster, implying that they are sourced from short-lived plasma cells (28) (fig. S14B).

Class III psNb (2-10) can destabilize the spike in vitro as we observed previously (16). However, the structure can be resolved by reconstituting an RBD: 4 Nbs complex by cryoEM (Methods, fig. S13). 2-10 targets a rare non-RBS epitope that partially overlaps with that of $\mathrm{Nb17}$ (Fig. 5E) isolated from the initial bleed that is partially effective against variants (16). Class III psNb binds strongly to RBD variants and clade $1 \mathrm{~b}$ and shows selectivity within other clades. Compared to $\mathrm{Nb} 17$ with limited breadth, the improved breadth of class III ps $\mathrm{Nb}$ is contributed by a recognition motif that shifts (from aa 356-357 to aa 449-450,481-484 and 490-494) towards a smaller and more conserved epitope (Fig. 5F, fig. S15B).

Four Class IV psNbs (1-22, 1-23, 2-65 and 2-45) were resolved by cryoEM. Their binding involves a plethora of molecular contacts that are dependent upon distinct scaffold orientations, different CDR combinations and sequence-specific bonding networks. Class IV shares a highly conserved and cryptic epitope that is only accessible in the RBD-up conformation (Fig. 3, A and C, Fig. 5G). The epitope is characterized by charged and hydrophobic residues (D427/D428, T430, F464, ${ }_{516} E_{L L H}{ }_{519}$ ) forming a cavity (Fig. 5G). CDR3s of class IV adopt a "hairpin" conformation that inserts into the RBD cavity forming extensive hydrophobic and polar interactions. 1-22 and 1-23 are all-clade binders that recognize multiple other conserved RBD residues (L390, P426 and P463 for 1-22 and Y369 for 1-23) through hydrophobic interactions (fig. S15, C and D). 2-65 shows selectivity towards clade $1 \mathrm{~b}$ and clade 3 likely due to a unique salt bridge between D124 (CDR3) and the semi-conserved H519 on RBDs (fig. S15E). Notably, the class IV epitope partially overlaps with a newly discovered broadly neutralizing antibody $\mathrm{S} 2 \mathrm{H} 97(10)$ and targets RBD from a distinct angle (fig. S16). Compared to S2H97 which depends on bulky aromatic side chains (W, F, and $\mathrm{Y}$ ) for interaction, the binding of 1-22 is primarily mediated through a combination of hydrophobic, non-aromatic ( $L, I$, and $V)$, and basic ( $R$ and $\mathrm{H})$ residues. Facilitated by a unique orientation, Class V psNb (2-62, which shares high CDR similarity with 263) targets RBD through a conserved epitope (T333,L387, ${ }_{389} \mathrm{DLC}_{391}, \mathrm{C} 525, \mathrm{~L} 517$, and P521) completely buried in the spike and a region that marginally overlaps with the binding motif of class IV psNbs (i.e., L517 and H519) (Fig. 5, H and I). While 2-62 binds tightly to the RBD, it does not bind the pre-fusion spike and is therefore a poor neutralizer of the virus (fig. S6).

\section{Mechanisms of broad neutralization by psNbs}

Our comprehensive investigation provides direct evidence that with successive immunization, antibodies emerged in the serum that concurrently and almost exhaustively target diverse and conserved physicochemical and geometric sites on the RBD. We observed that ultrahigh-affinity Nbs (i.e., sub-nM) exhibit neutralization potencies that correlate inversely, almost perfectly, with distance of their epitopes from the RBS (Fig. 6, A and B, Methods). Ultrapotent class I psNbs neutralize the virus at single-digit ng/ml by binding to the RBS to block hACE2 binding (fig. S17). Class II psNbs can still efficiently neutralize the virus (i.e., singledigit $\mathrm{ng} / \mathrm{ml}$ ) primarily by sterically interfering with the receptor binding. In particular, binding to the RBD can 
clash with the glycan moiety at N322 of hACE2, especially bulky complex-type glycans (29) (fig. S17). Class III, IV and V possess substantially weaker potencies. Their epitopes are distant from RBS and do not compete with hACE2 in vitro.

To better understand the broad activities of psNbs, we also expressed a set of high-affinity RBD Nbs that have been previously characterized (18-25) and evaluated cross-reactivity by ELISA (table S4, Methods). $\mathrm{Nbs}$ fall into two groups based on RBS occupancy. Overall, their cross-reactivities positively correlate with epitope sequence conservation (Fig. 6C). psNbs that do not bind the RBS can be separated based on high epitope conservation (>0.85) (Fig. 6D). Critical mutations from the variants are predominantly located on the RBS but also recently on a small patch of relatively conserved residues (e.g., aa 371, 373 and 375) in Omicron (B.1.1.529). Additionally, the virus can mutate on A372S/T to acquire an N-linked glycan (N370) similar to other sarbecoviruses to blunt the host antibody response (30). While most $\mathrm{Nbs}$ and human IgGs directly interact with these residues, psNbs are barely affected (Fig. 2A-B, fig. S5). Mutations on these highly conserved epitopes can cause RBD instability reducing viral fitness (31) (Fig. 6E).

Compared to RBS Nbs that bind to concave epitopes, psNbs generally target smaller (Fig. $6 \mathrm{H}$ ) and flat (class II,III and V) or convex (class I and IV) regions (Fig. 6F). psNb epitopes are also more flexible based on average root-mean-square-fluctuation (RMSF) for epitope residues (Fig. 6G, table S5, Methods). Together, these properties may render high-affinity binding particularly challenging. Consistently, ScanNet, a geometric deep-learning model reveals that RBS epitopes are predominantly targeted by Nbs (and lgG antibodies) while the psNb epitopes are hardly recognized (fig. S19). Moreover, compared to non-psNbs, psNbs utilize almost exclusively hypervariable CDR loops (fig. S18). Conceivably, extensive affinity maturation is required to bind these conserved yet difficult-to-bind epitopes.

\section{Discussion}

SARS-CoV-2 continues to evolve, producing variants with high transmissibility and potential to evade host immunity $(32,33)$. New evidence indicates that infection or vaccination by boosting can improve the host antibody response against SARS-CoV-2 variants (3-7). Here we discovered that successive immunization of a camelid by recombinant RBD can enhance the development of super-immunity. Integrative proteomics facilitated rapid identification of a large repertoire of high-affinity $\mathrm{V}_{\mathrm{H}} \mathrm{H}$ antibodies $(\mathrm{Nbs})$ from the immunized sera against SARS-CoV-2 VOCs and the full spectrum of sarbecovirus family. CryoEM and X-ray crystallography were able to systematically map broadly neutralizing epitopes and interactions, providing insights into the structural basis and evolution of serologic activities. Our data support the notion that RBD structure alone can drive this impressive level of evolution, reshaping the immunogenic landscape towards conserved epitopes. The initial immune response predominantly targets the RBS due to its favorable properties for protein-protein interactions (both host receptor and antibody binding). High-affinity antibodies can evolve to saturate this critical region with extremely high neutralization potency. However, broadly neutralizing antibodies will emerge (with unprecedented diversity) and can continuously develop improved affinity to target conserved epitopes, 
which provide resistance to antigenic drift. Their neutralization potencies vary substantially yet are strongly and negatively correlated with the distance of epitopes to the RBS-center for viral entry and effective host immunity. Together, our findings inform on the development of safe and broadly protective countermeasures such as vaccines and therapeutics.

Preference for non-conserved epitopes is also observed in other viral antigen structures (fig. S20). We suggest that evolutionary conservation imposes constraints on spatio-chemical surface properties, which in turn constraints the immunogenicity of and access to epitopes, as hinted by an overall negative correlation between conservation and predicted epitope propensity (fig. S21). However, antibody repertoires are dynamic and can evolve towards conserved epitopes. In addition to COVID-19 super-immunity, potent broadly neutralizing antibodies have also been isolated from HIV elite controllers, although they usually take years to evolve (34).

Our highly selected psNbs can bind strongly and specifically to all sarbecoviruses for potent neutralization with the best median antiviral potencies at single-digit $\mathrm{ng} / \mathrm{ml}$, which is extremely rare for crossreactive antibodies (35). Multivalent constructs (such as $\mathrm{PiN}-31$ ) that target distinct and conserved epitopes and their cocktails may provide comprehensive coverage against future SARS-CoV-2 antigenic drift and new sarbecovirus challenges. The low production costs and marked stability of $\operatorname{Nbs}(19,23,36-38)$ (and other miniproteins (39) allow for more equitable and efficient distributions globally, particularly for developing countries and regions that are vulnerable to viral spillovers. Combined with their small size (high effective dose and bioavailability) and flexible administration routes that protect both upper and lower respiratory tracts to limit airborne transmissions (40), ultrapotent and inhalable psNbs are therefore highly complementary to vaccines, small molecule drugs, and monoclonal antibody therapeutics $(41,42)$. The prospects of winning the race against future outbreaks will rely on the fast development and equitable distribution of an arsenal of broadly protective, cost-effective and convenient technologies. 


\section{REFERENCES AND NOTES}

1. R. M. Meganck, R. S. Baric, Developing therapeutic approaches for twenty-first-century emerging infectious viral diseases. Nat Med 27, 401-410 (2021).

2. M. F. Boni et al., Evolutionary origins of the SARS-CoV-2 sarbecovirus lineage responsible for the COVID-19 pandemic. Nat Microbiol 5, 1408-1417 (2020).

3. E. Callaway, COVID super-immunity: one of the pandemic's great puzzles. Nature 598, 393-394 (2021).

4. F. Schmidt et al., High genetic barrier to SARS-CoV-2 polyclonal neutralizing antibody escape. Nature 600, 512-516 (2021).

5. A. Cho et al., Anti-SARS-CoV-2 receptor-binding domain antibody evolution after mRNA vaccination. Nature 600, 517-522 (2021).

6. L. Stamatatos et al., mRNA vaccination boosts cross-variant neutralizing antibodies elicited by SARSCoV-2 infection. Science, eabg9175 (2021).

7. Z. Wang et al., Naturally enhanced neutralizing breadth against SARS-CoV-2 one year after infection. Nature 595, 426-431 (2021).

8. L. Piccoli et al., Mapping neutralizing and immunodominant sites on the SARS-CoV-2 spike receptorbinding domain by structure-guided high-resolution serology. Cell 183, 1024-1042 e1021 (2020).

9. C. G. Rappazzo et al., Broad and potent activity against SARS-like viruses by an engineered human monoclonal antibody. Science 371, 823-829 (2021).

10. T. N. Starr et al., SARS-CoV-2 RBD antibodies that maximize breadth and resistance to escape. Nature 597, 97-102 (2021).

11. D. R. Martinez et al., A broadly cross-reactive antibody neutralizes and protects against sarbecovirus challenge in mice. Sci Transl Med, eabj7125 (2021).

12. D. Pinto et al., Cross-neutralization of SARS-CoV-2 by a human monoclonal SARS-CoV antibody. Nature 583, 290-295 (2020).

13. M. A. Tortorici et al., Broad sarbecovirus neutralization by a human monoclonal antibody. Nature 597, 103-108 (2021).

14. Y. Xiang et al., Versatile and multivalent nanobodies efficiently neutralize SARS-CoV-2. Science 370, 1479-1484 (2020).

15. Y. Xiang et al., Integrative proteomics identifies thousands of distinct, multi-epitope, and high-affinity nanobodies. Cell Syst 12, 220-234 e229 (2021).

16. D. Sun et al., Potent neutralizing nanobodies resist convergent circulating variants of SARS-CoV-2 by targeting diverse and conserved epitopes. Nat Commun 12, 4676 (2021).

17. A. C. Walls et al., Structure, function, and antigenicity of the SARS-CoV-2 spike glycoprotein. Cell 181, 281-292 e286 (2020).

18. J. Xu et al., Nanobodies from camelid mice and llamas neutralize SARS-CoV-2 variants. Nature 595, 278-282 (2021).

19. P. A. Koenig et al., Structure-guided multivalent nanobodies block SARS-CoV-2 infection and suppress mutational escape. Science 371, eabe6230 (2021).

20. J. Huo et al., A potent SARS-CoV-2 neutralising nanobody shows therapeutic efficacy in the Syrian golden hamster model of COVID-19. Nat Commun 12, 5469 (2021).

21. T. R. Wagner et al., NeutrobodyPlex-monitoring SARS-CoV-2 neutralizing immune responses using nanobodies. EMBO Rep 22, e52325 (2021).

22. G. Ye et al., The development of Nanosota-1 as anti-SARS-CoV-2 nanobody drug candidates. eLife 10, e64815 (2021).

23. P. Pymm et al., Nanobody cocktails potently neutralize SARS-CoV-2 D614G N501Y variant and protect mice. Proc Natl Acad Sci U S A 118, e2101918118 (2021).

24. J. Huo et al., Neutralizing nanobodies bind SARS-CoV-2 spike RBD and block interaction with ACE2. Nat Struct Mol Biol 27, 846-854 (2020). 
25. T. Guttler et al., Neutralization of SARS-CoV-2 by highly potent, hyperthermostable, and mutationtolerant nanobodies. EMBO J 40, e107985 (2021).

26. Y. Watanabe et al., Vulnerabilities in coronavirus glycan shields despite extensive glycosylation. Nat Commun 11, 2688 (2020).

27. J. Lan et al., Structure of the SARS-CoV-2 spike receptor-binding domain bound to the ACE2 receptor. Nature 581, 215-220 (2020).

28. M. Akkaya, K. Kwak, S. K. Pierce, B cell memory: building two walls of protection against pathogens. Nat Rev Immunol 20, 229-238 (2020).

29. A. R. Mehdipour, G. Hummer, Dual nature of human ACE2 glycosylation in binding to SARS-CoV-2 spike. Proc Natl Acad Sci U S A 118, (2021).

30. K. G. Nabel et al., Structural basis for continued antibody evasion by the SARS-CoV-2 receptor binding domain. Science, eabl6251 (2021).

31. T. N. Starr et al., Deep mutational scanning of SARS-CoV-2 receptor binding domain reveals constraints on folding and ACE2 binding. Cell 182, 1295-1310.e20 (2020).

32. W. T. Harvey et al., SARS-CoV-2 variants, spike mutations and immune escape. Nat Rev Microbiol 19, 409-424 (2021).

33. S. S. A. Karim, Q. A. Karim, Omicron SARS-CoV-2 variant: a new chapter in the COVID-19 pandemic. Lancet 398, 2126-2128 (2021).

34. D. Sok, D. R. Burton, Recent progress in broadly neutralizing antibodies to HIV. Nat Immunol 19, 11791188 (2018).

35. L. M. Walker, D. R. Burton, Passive immunotherapy of viral infections: 'super-antibodies' enter the fray. Nat Rev Immunol 18, 297-308 (2018).

36. S. Muyldermans, Nanobodies: natural single-domain antibodies. Annu Rev Biochem 82, 775-797 (2013).

37. F. D. Mast et al., Highly synergistic combinations of nanobodies that target SARS-CoV-2 and are resistant to escape. eLife 10, e73027 (2021).

38. M. Schoof et al., An ultrapotent synthetic nanobody neutralizes SARS-CoV-2 by stabilizing inactive Spike. Science 370, 1473-1479 (2020).

39. L. Cao et al., De novo design of picomolar SARS-CoV-2 miniprotein inhibitors. Science 370, 426-431 (2020).

40. S. Nambulli et al., Inhalable Nanobody (PiN-21) prevents and treats SARS-CoV-2 infections in Syrian hamsters at ultra-low doses. Sci Adv 7, eabh0319 (2021).

41. C. Liu et al., Research and Development on Therapeutic Agents and Vaccines for COVID-19 and Related Human Coronavirus Diseases. ACS Cent Sci 6, 315-331 (2020).

42. M. Cully, A tale of two antiviral targets - and the COVID-19 drugs that bind them. Nat Rev Drug Discov, (2021).

\section{ACKNOWLEDGEMENT}

We thank P.J. Bjorkman (Caltech) for sharing plasmids bearing sarbecovirus RBDs, the UPMC Genome Center for Illumina MiSeq, and Yong Joon Kim for proofreading of the manuscript. We thank Wenli Yu, Henry Tien, Xueyong Zhu, Meng Yuan, and Robyn L. Stanfield, from the Wilson lab for help with insect cell culture, crystal screening, and X-ray data collection. Funding: This work was supported by the University of Pittsburgh School of Medicine (Y.S.), NIH grants R35GM137905 (Y.S.), R01GM133841 (D.J.T.) and the Bill and Melinda Gates Foundation INV-004923 (I.A.W.). A portion of this research was supported by NIH grant U24GM129547 and performed at the PNCC at Oregon Health \& Science University and accessed through the Environmental Molecular Sciences Laboratory (grid.436923.9), a Department of Energy, Office of Science User Facility sponsored by the Office of Biological and Environmental Research, ISF 1466/18 and Israeli Ministry of Science 
and Technology (D.S), the Edmond J. Safra Center for Bioinformatics at Tel Aviv University and from the Human Frontier Science Program (cross-disciplinary postdoctoral fellowship LT001058/2019-C) (JT). This research used resources of the Advanced Photon Source, a U.S. Department of Energy (DOE) Office of Science User Facility, operated for the DOE Office of Science by Argonne National Laboratory under Contract No. DE-AC02-06CH11357. Extraordinary facility operations were supported in part by the DOE Office of Science through the National Virtual Biotechnology Laboratory, a consortium of DOE national laboratories focused on the response to COVID-19, with funding provided by the Coronavirus CARES Act. Author contributions: Y.S. conceived the study. Y.X. identified and characterized Nbs. Z.S. developed scripts and analyzed structures. W.H. and D.J.T. solved cryoEM structures. H.L. and I.A.W. determined and analyzed Xray crystallographic structures. J.T. and D.S. analyzed viral antigenicity. S.N. and P.W.D performed the PRNT assay. Y.S and Y.X. drafted the manuscript with substantial input from I.A.W., W.H., Z.S., D.S., H.L., and D.J. T. All authors reviewed the manuscript. We thank D.S.Reed (University of Pittsburgh) and N.A.Crossland (Boston University) for technical assistance. Competing interests: Y.S. and Y.X. are co-inventors on a provisional patent filed by the University of Pittsburgh covering the Nbs herein described. Data and material availability: Plasmids of psNbs can be requested from Y.S. (yi.shi@pitt.edu).

\section{SUPPLEMENTARY MATERIALS}

\section{Materials and Methods}

Figs. $\mathrm{S1}$ to $\mathrm{S21}$

Tables S1-S5

References 43-86

\section{Figures}


bioRxiv preprint doi: https://doi.org/10.1101/2021.12.26.474192; this version posted December 28, 2021. The copyright holder for this preprint (which was not certified by peer review) is the author/funder, who has granted bioRxiv a license to display the preprint in perpetuity. It is made available under aCC-BY-NC-ND 4.0 International license.

\section{Figure 1}

A

B

Clade $1 \mathrm{a}$

B
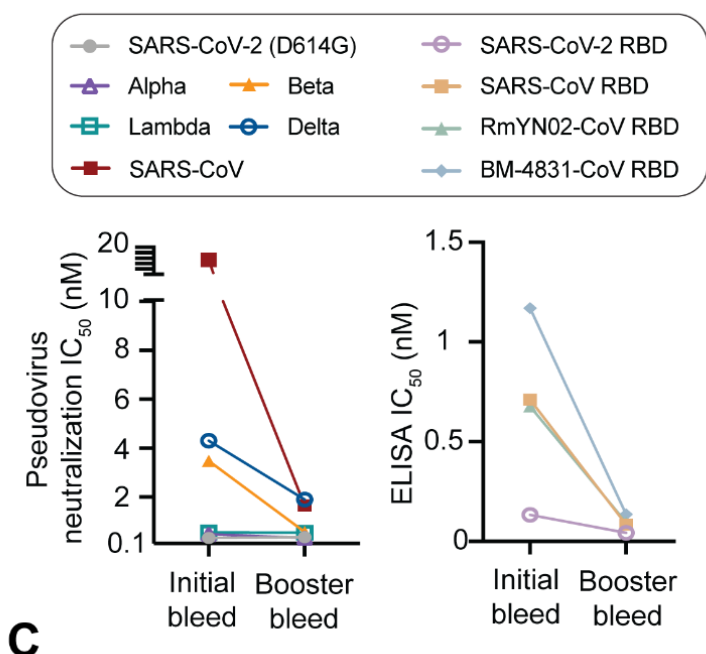

C

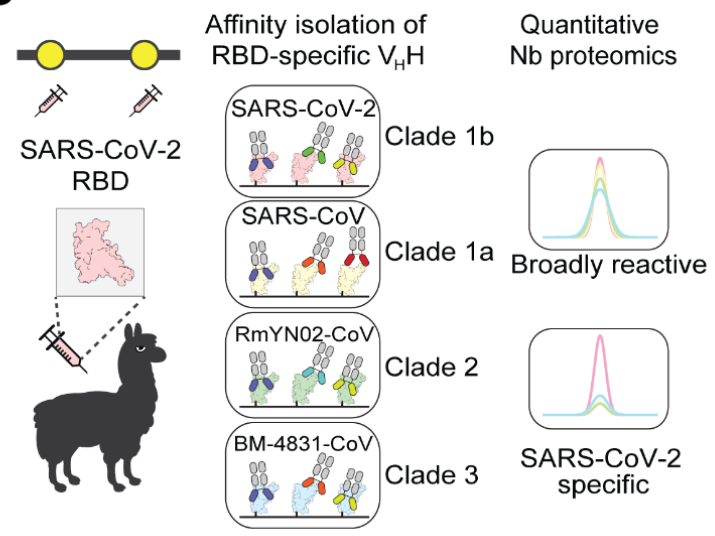

Clade 2

Clade 3
D
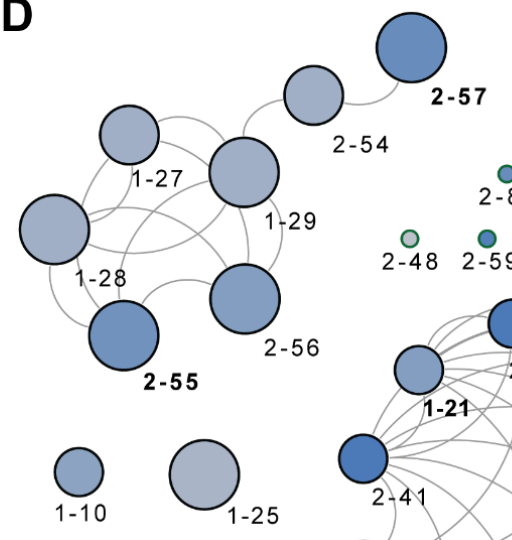

$$
\begin{array}{cccc} 
& \multicolumn{4}{c}{0} \\
& & & \\
2-47 \\
& 0 & 0 & 0 \\
2-69 & 2-58 & 2-50 \\
2-8 & 0 & \circ & 0 \\
2-8 & 1-24 & 2-9 & 1-30
\end{array}
$$

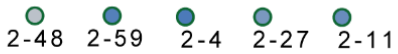
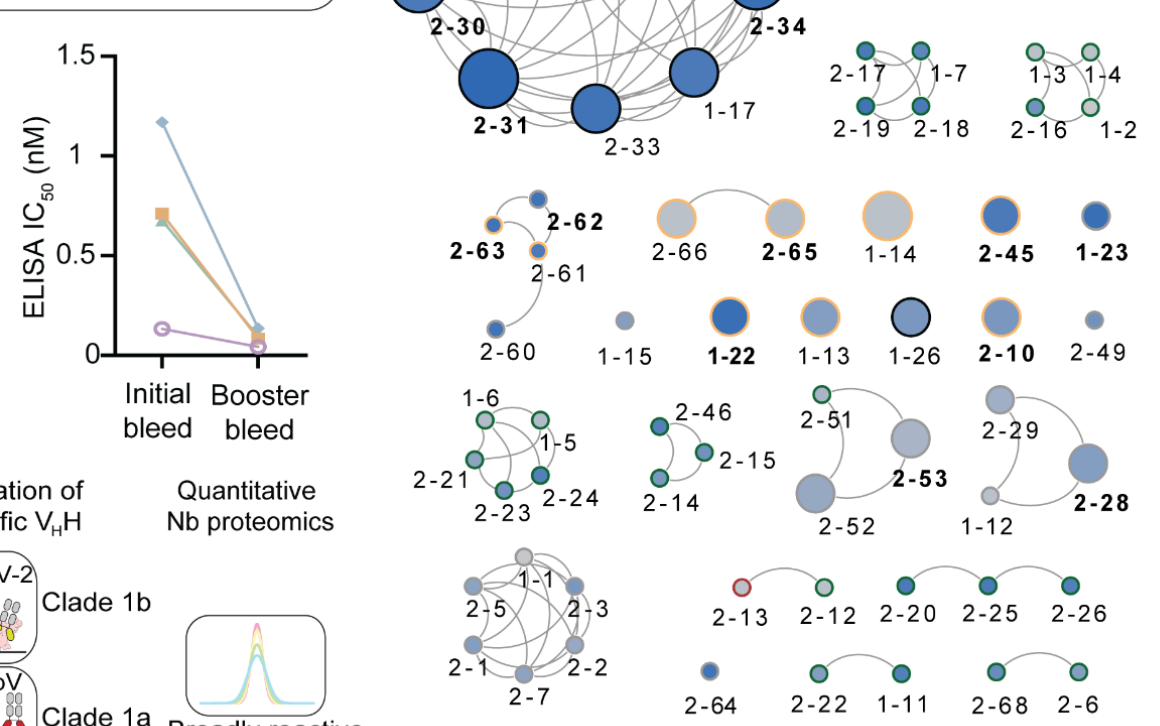

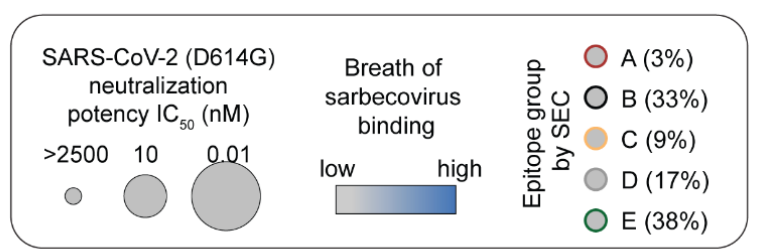


Fig. 1. Identification and characterization of psNbs. (A) Phylogenetic tree of 19 RBDs from all 4 clades of sarbecoviruses, constructed by the maximum likelihood. (B) The neutralization of polyclonal $\mathrm{V}_{\mathrm{H}} \mathrm{Hs}$ from two immunization bleeds against pseudovirus of SARS-CoV-2, its variants and SARS-CoV. Their ELISA IC ${ }_{50} S$ against 4 RBD clades were also shown. (C) Schematics for proteomic identification of psNbs from immunized sera. (D) A map summarizing RBD binding and neutralization for 100 high-affinity psNbs. Nbs are represented by dots of various sizes and colors. Two $\mathrm{Nbs}$ are connected if their CDR sequence identity is $>70 \%$. Their neutralization potencies against pseudotyped SARS-CoV-2 (D614G) are represented by the size of dots. Breadth of sarbecovirus RBD binding is indicated by the filled gradient color (table S1) and the SEC epitope groups (based on benchmark RBD Nbs) are colored on the outer circle (fig. S3, a and b). The highest Nb concentration used for binding and neutralization was $8 \mu \mathrm{M}$ and $2.5 \mu \mathrm{M}$, respectively. Bolded psNbs were selected for downstream characterizations. psNbs isolated from the initial and booster bleed were denoted as 1-, and 2- respectively. 
bioRxiv preprint doi: https://doi.org/10.1101/2021.12.26.474192; this version posted December 28, 2021. The copyright holder for this preprint (which was not certified by peer review) is the author/funder, who has granted bioRxiv a license to display the preprint in perpetuity. It is made available under aCC-BY-NC-ND 4.0 International license.

\section{Figure 2}

A

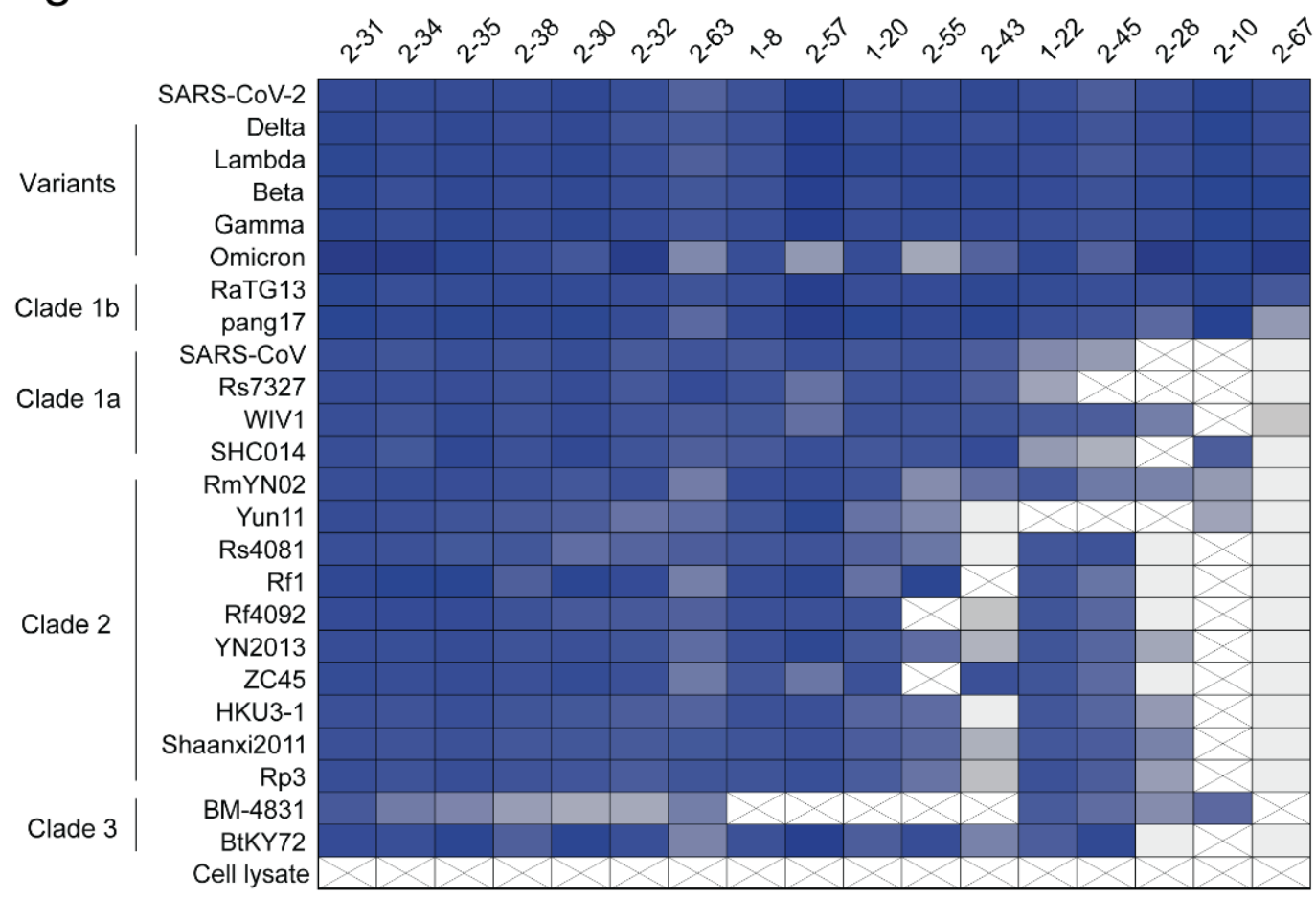

B

\begin{tabular}{|c|c|c|c|c|c|c|c|c|c|c|c|c|c|c|c|c|c|c|}
\hline & D614G & 5.12 & 4.05 & 7.59 & 6.63 & 9.09 & 7.27 & $\mathrm{~N} / \mathrm{A}$ & 11.8 & 0.90 & 6.43 & 0.45 & 12.8 & 252 & 104 & 482 & 140 & 0.08 \\
\hline & Alpha & 3.44 & 1.38 & 18.4 & 3.29 & 3.82 & 15.3 & $\mathrm{~N} / \mathrm{A}$ & 11.4 & 1.67 & 6.35 & 0.41 & 10.9 & 36.8 & 101 & 2067 & 26.0 & 0.08 \\
\hline & Beta & 4.66 & 3.61 & 4.88 & 4.63 & 6.61 & 8.76 & $\mathrm{~N} / \mathrm{A}$ & 1.99 & 2.41 & 6.60 & 0.47 & 7.70 & 2115 & 98.9 & 514 & 95.8 & 1.89 \\
\hline & Lambda & 3.60 & 1.32 & 3.35 & 5.30 & 6.52 & 5.95 & N/A & 3.73 & 2.95 & 12.7 & 0.53 & 5.99 & 26.2 & 96.0 & 538 & 73.0 & 0.07 \\
\hline & Delta & 3.53 & 5.52 & 3.74 & 12.8 & 5.64 & 9.07 & N/A & 10.6 & 4.90 & 5.07 & 0.51 & 5.33 & 110 & 107 & 394 & 133 & 0.18 \\
\hline & SARS-CoV & 4.18 & 2.40 & 2.28 & 2.23 & 3.54 & 6.58 & N/A & 564 & 11.3 & 5.40 & 0.19 & 77.2 & 6931 & $\mathrm{~N} / \mathrm{A}$ & N/A & N/A & N/A \\
\hline
\end{tabular}

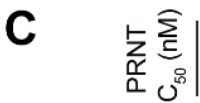

\begin{tabular}{c|c|c|c|c|c|c|c|c|c|c|c|c|c|c|c|c|c|} 
Munich & 7.41 & 42.2 & 19.1 & 59.9 & 10.6 & 32.2 & 8657 & 8.36 & 9.79 & 13.1 & 0.36 & 44.5 & 1106 & 42.8 & 410 & 264 & 0.35 \\
\cline { 2 - 12 } Delta & 3.62 & 21.0 & 9.16 & 34.7 & 27.2 & 8.76 & 8913 & 40.2 & 8.74 & 9.54 & 2.25 & 36.5 & 146 & 211 & 313 & 171 & 0.31 \\
\cline { 2 - 8 }
\end{tabular}

\begin{tabular}{|llll}
\hline $\begin{array}{c}\text { ELISA } \\
\text { binding }\end{array}$ & $\square$ Not determined & $\square$ 5-fold improvement \\
$\mathrm{IC}_{50}(\mathrm{nM})$ & $\mathrm{N}^{3}, 0^{2}, 0^{\circ}, 0^{\circ}$ & No binding $(8 \mu \mathrm{M})$ & $\square>$-fold decrease \\
\hline
\end{tabular}

D

\section{2-31}

SARS-CoV-2 RBD

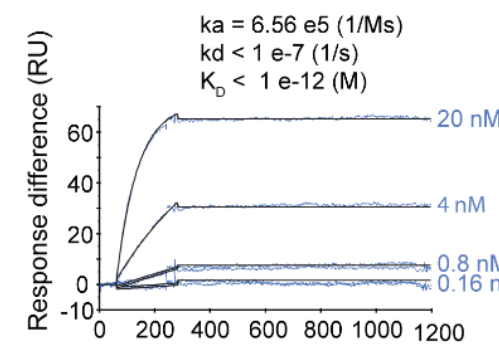

E

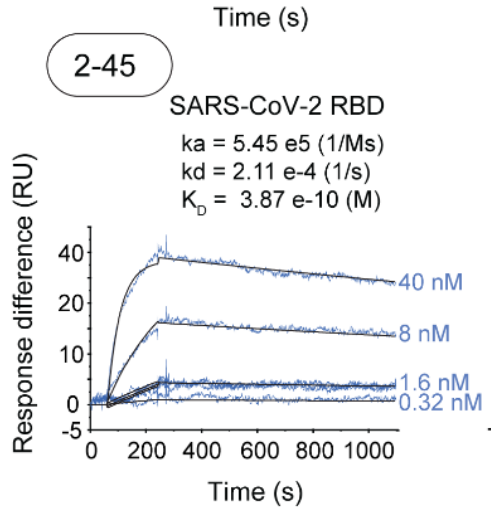

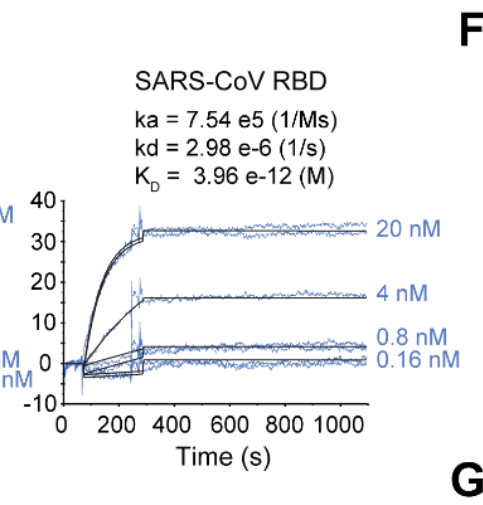

BM-4831 RBD

$k a=2.94$ e5 (1/Ms)

$k d=1.29 \mathrm{e}-5(1 / \mathrm{s})$

$\mathrm{K}_{\mathrm{D}}=4.41 \mathrm{e}-11(\mathrm{M})$

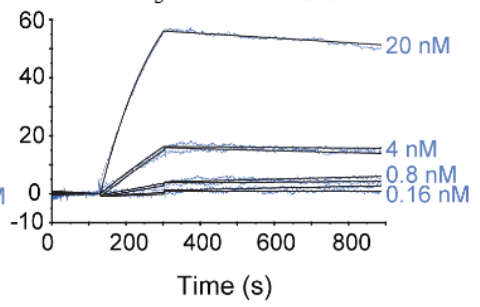

$\mathbf{F}$

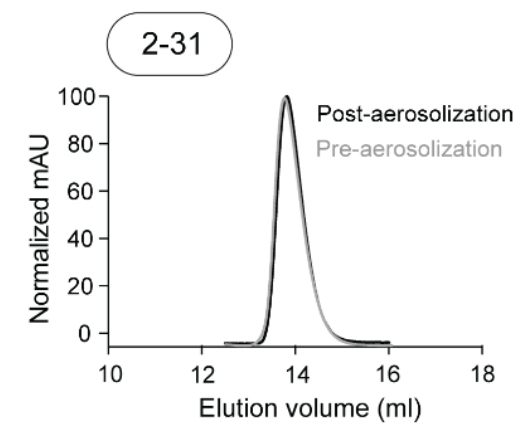

G
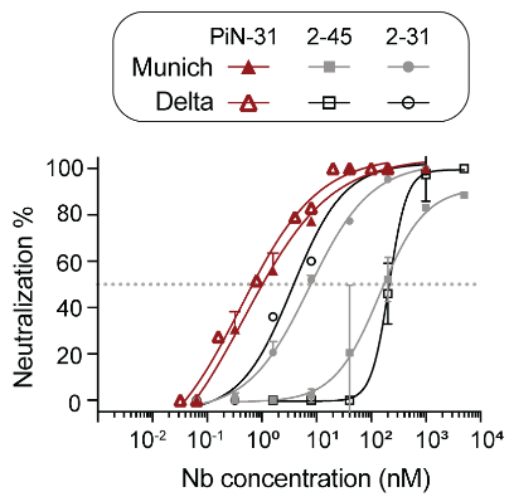
Fig. 2. Binding and neutralization of $17 \mathrm{psNbs}$ and development of a bispecific and inhalable ps $\mathrm{Nb}$

(PiN-31). (A) Heatmap summary of psNbs for binding to different RBDs by ELISA. White with cross mark: no binding. Silver: binding signals were detected at $8 \mu \mathrm{M}$ yet the $\mathrm{IC}_{50}$ s have not been determined. Blue shades: $\mathrm{IC}_{50}$ between $0.8 \mathrm{nM}$ to $8 \mu \mathrm{M}$. (B) Neutralization potency of psNbs against pseudotyped SARS-CoV-2, its variants and SARS-CoV. N/A: no neutralization detected at the highest $\mathrm{Nb}$ concentration $(2.5 \mu \mathrm{M})$. (C) (C) Neutralization potency against SARS-CoV-2 Munich strain and the Delta VOC by the PRNT assay. (D) Binding kinetic measurements of 2-31 for RBD sARs-Cov-2 and RBD $_{\text {sars-cov }}$ by surface plasmon resonance (SPR). (E) SPR

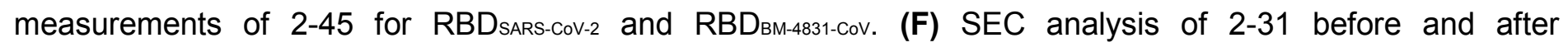
aerosolization with soft mist inhaler. (G) Neutralization potency of a bispecific psNb construct (PiN-31) by the PRNT assay. 
bioRxiv preprint doi: https://doi.org/10.1101/2021.12.26.474192; this version posted December 28, 2021. The copyright holder for this preprint (which was not certified by peer review) is the author/funder, who has granted bioRxiv a license to display the preprint in perpetuity. It is made available under aCC-BY-NC-ND 4.0 International license.

\section{Figure 3}

\section{A}

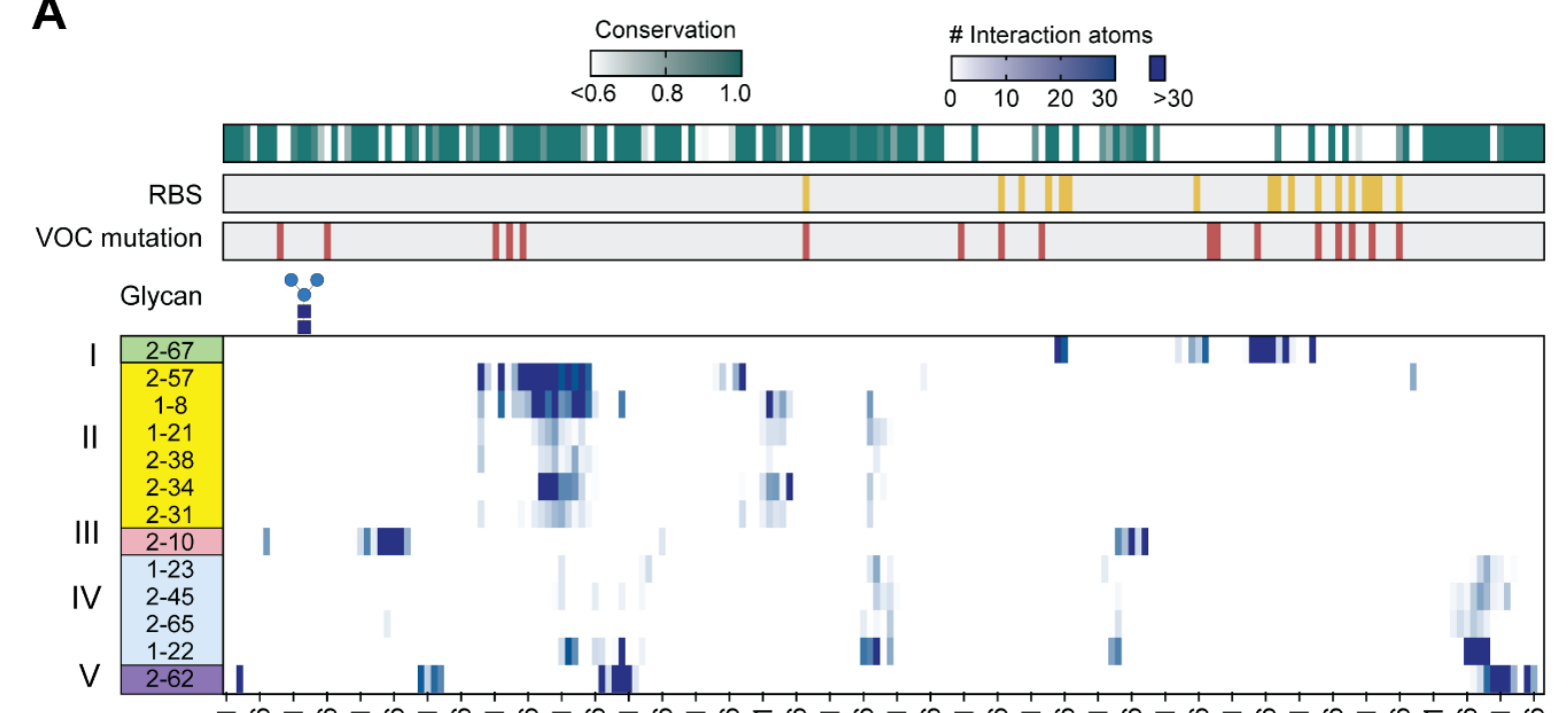

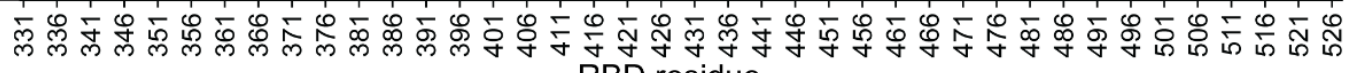

B

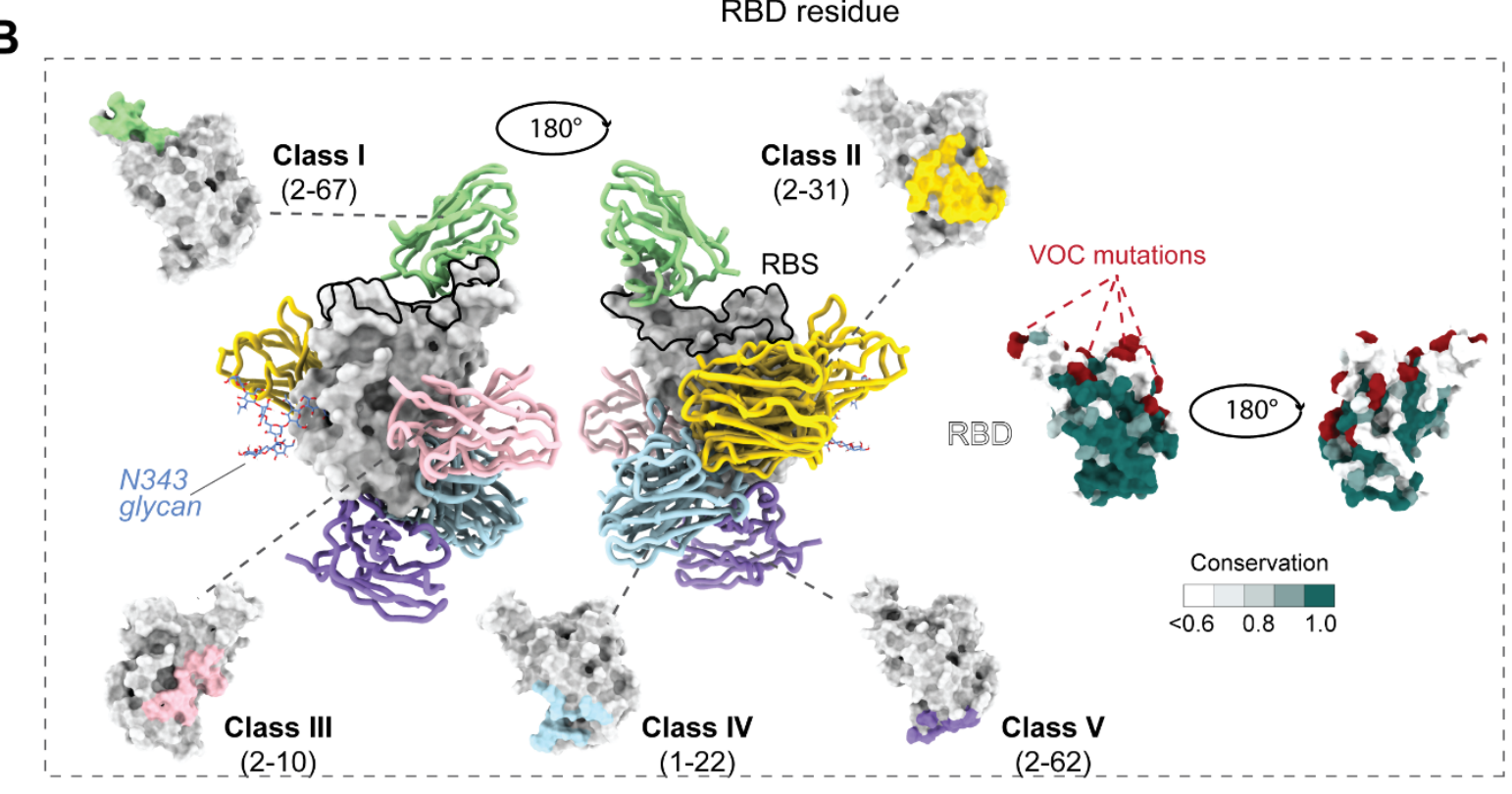

C

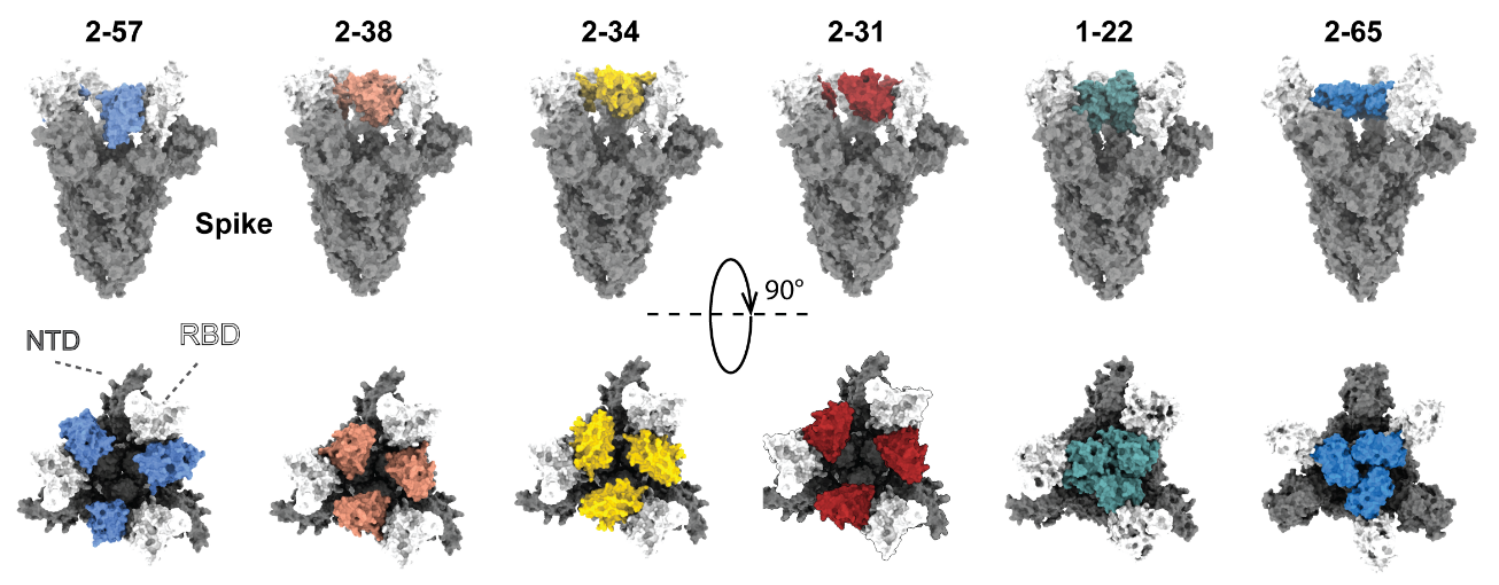


bioRxiv preprint doi: https://doi.org/10.1101/2021.12.26.474192; this version posted December 28, 2021. The copyright holder for this preprint (which was not certified by peer review) is the author/funder, who has granted bioRxiv a license to display the preprint in perpetuity. It is made available under aCC-BY-NC-ND 4.0 International license.

Fig. 3. Summary of broadly neutralizing RBD epitopes and spike conformations. (A) Clustering analysis of psNbs epitopes. RBS residues are in yellow. The RBD glycosylation site (N343) is denoted. Sarbecovirus sequence conservation is shown in green gradient. The number of psNb: RBD interaction atoms per epitope residue is shown in blue shades. (B) Structural representations of five classes of psNbs in complex with RBD and the corresponding epitopes. Light green: class I (2-67), gold: class II (2-31), pink: class III (2-10), light blue: class IV (1-22) and purple: class V (2-62). VOC residues are in red. RBD glycosylation (N343) is in cornflower blue. The same set of perspective view was used for comparison between psNb epitopes and VOC mutation sites. (C) Structural diversity of psNbs in complex with the SARS-CoV-2 pre-fusion spike glycoprotein (by cryoEM). RBDs are shown in white, and non-RBD regions of spike in grey. 
bioRxiv preprint doi: https://doi.org/10.1101/2021.12.26.474192; this version posted December 28, 2021. The copyright holder for this preprint (which was not certified by peer review) is the author/funder, who has granted bioRxiv a license to display the preprint in perpetuity. It is made available under aCC-BY-NC-ND 4.0 International license.

\section{Figure 4}

A

Evolutionary distance 씨ㄹㅠㅠํํํํํ응

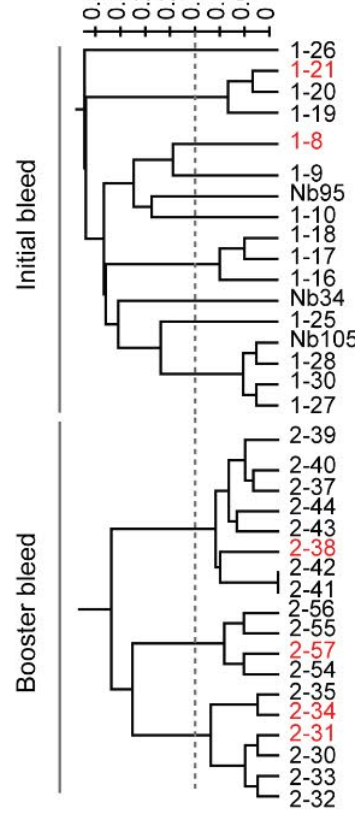

D Low
B

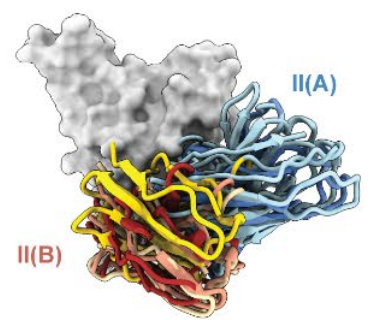

C
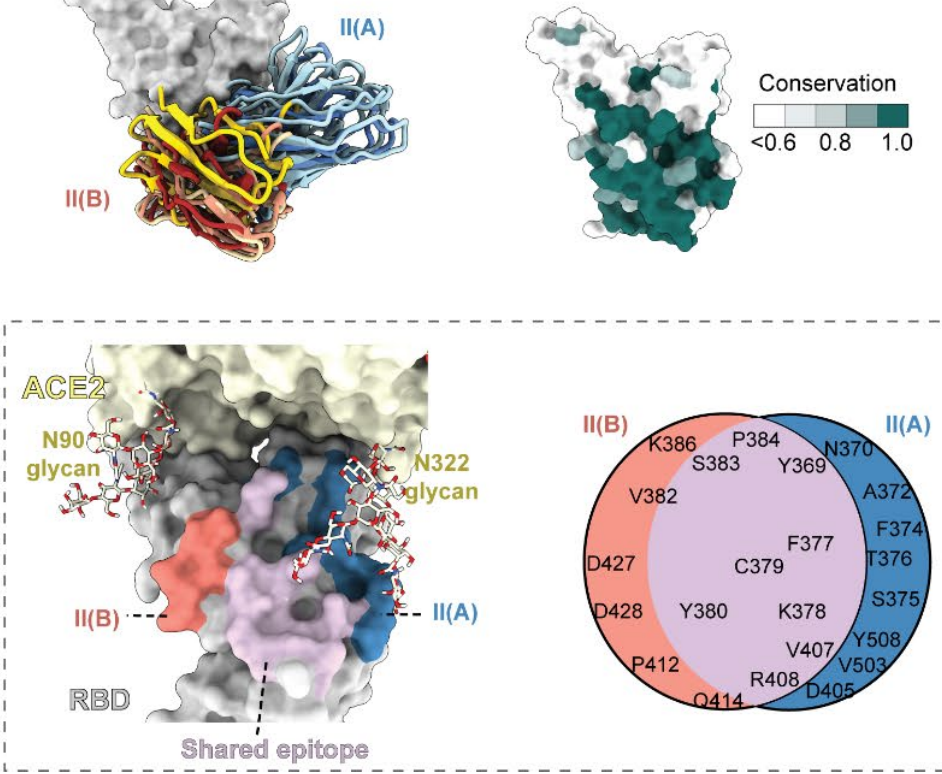

Breadth of sarbecovirus RBD binding

High
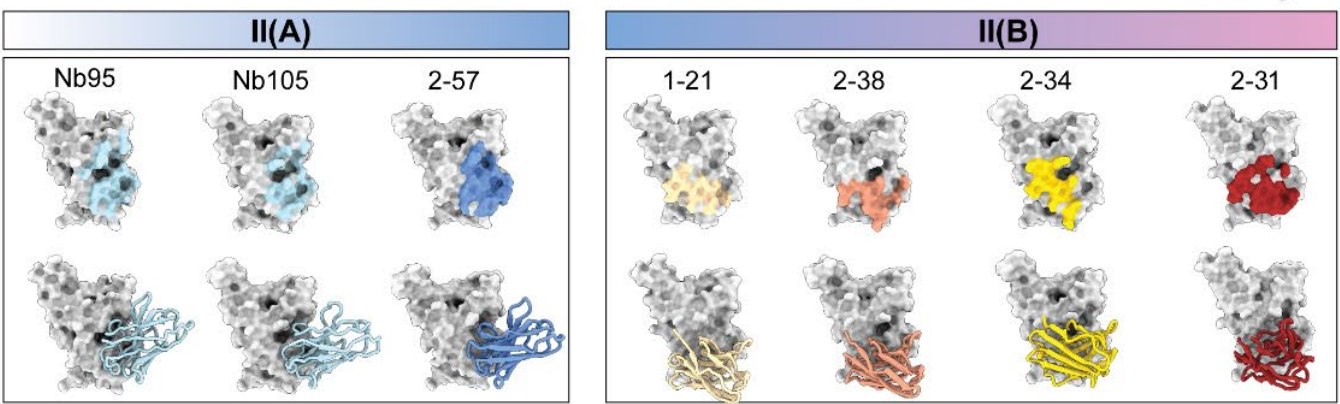

E

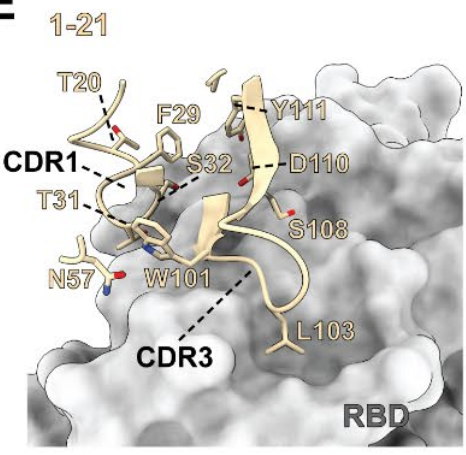

$2-38$

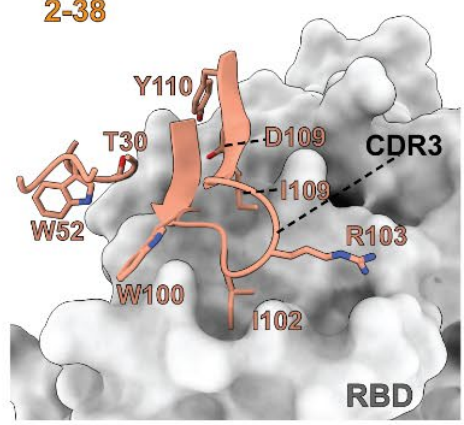

F

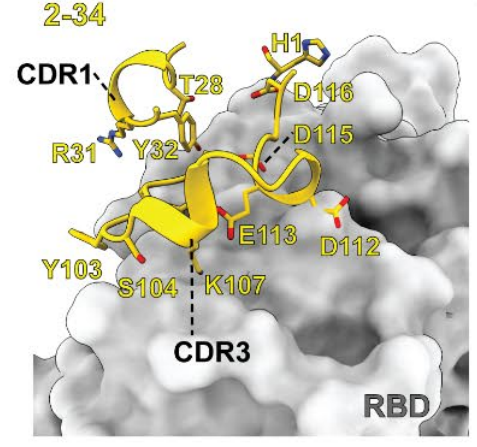

2-31

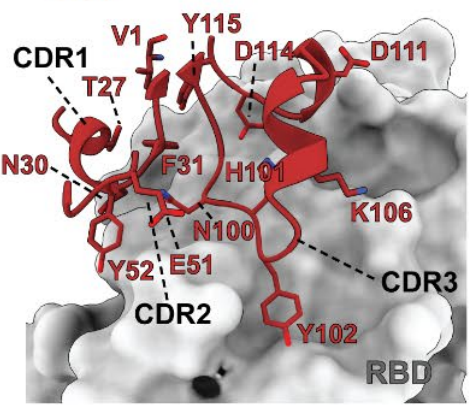

G

$\mathbb{N} b 105$

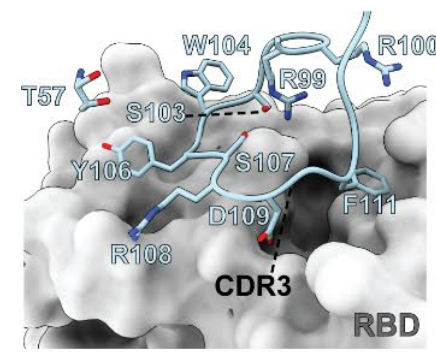

\section{$2=57$}

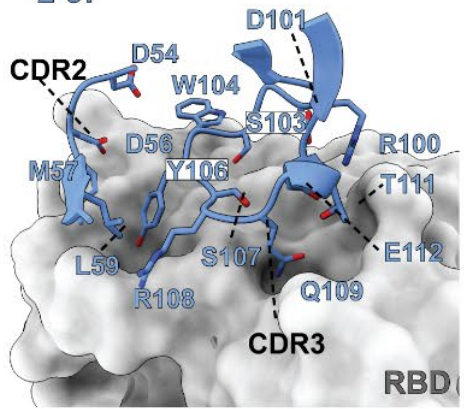


Fig. 4. Structural diversity, convergence and evolution of class II psNbs. (A) Phylogenetic analysis of psNbs from SEC group B. psNbs with structures determined in this study are in red. Scale of the evolutionary distance: 0- 0.35. (B) Superposition of class II psNbs reveals two subclasses II(A) and II(B). (C) Overlap between II(A) and II(B) epitopes. Purple: shared epitope; blue: non-overlapping epitope of II(A); salmon: nonoverlapping epitope of II(B); light yellow: hACE2 with modeled glycans (N90 and N322). (D) Structural overview of class II psNbs in complex with RBD and their epitopes. Light blue: Nb95 and Nb105; cornflower blue: 2-57; beige: 1-21; salmon: 2-38; gold: 2-34; dark red: 2-31. (E-G) Structural comparison of different Nb pairs (e) 1-21 and 2-38, (f) 2-34 and 2-31, and (g) Nb105 and 2-57 for RBD binding. 
bioRxiv preprint doi: https://doi.org/10.1101/2021.12.26.474192; this version posted December 28, 2021. The copyright holder for this preprint (which was not certified by peer review) is the author/funder, who has granted bioRxiv a license to display the preprint in perpetuity. It is made available under aCC-BY-NC-ND 4.0 International license.

\section{Figure 5}

A

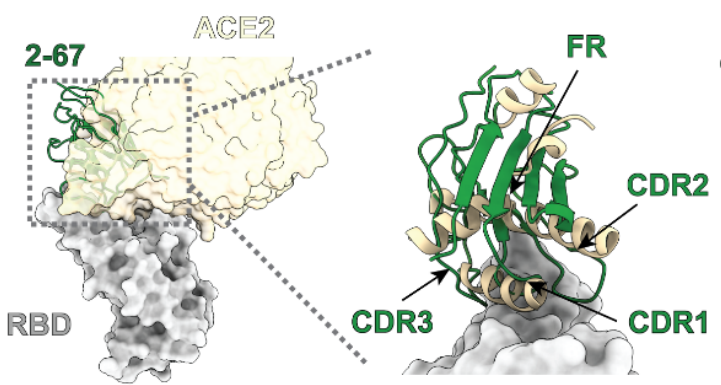

C

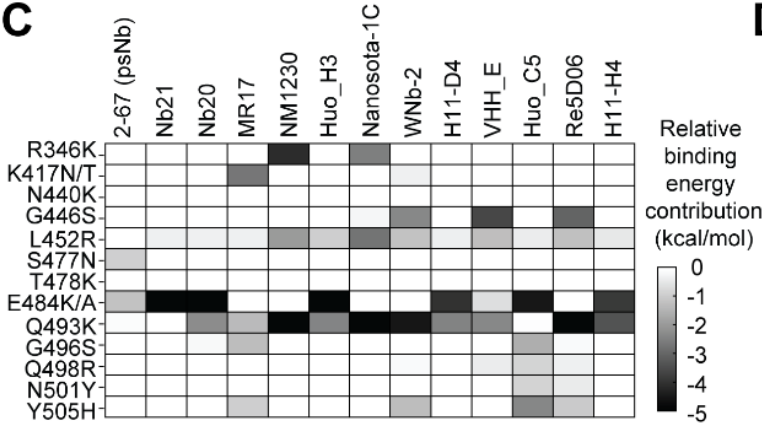

E

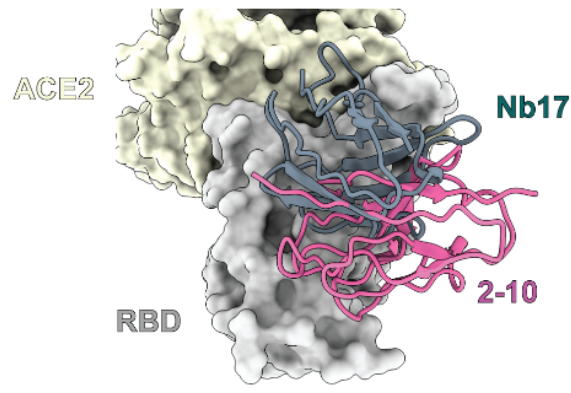

B

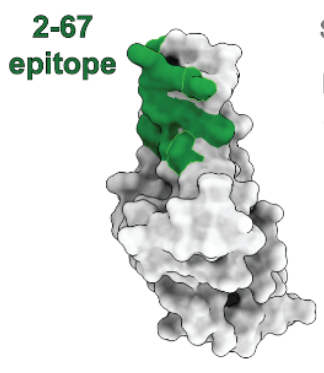

D

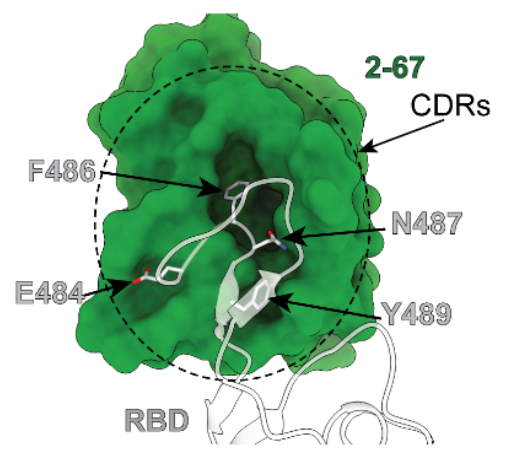

$\mathbf{F}$

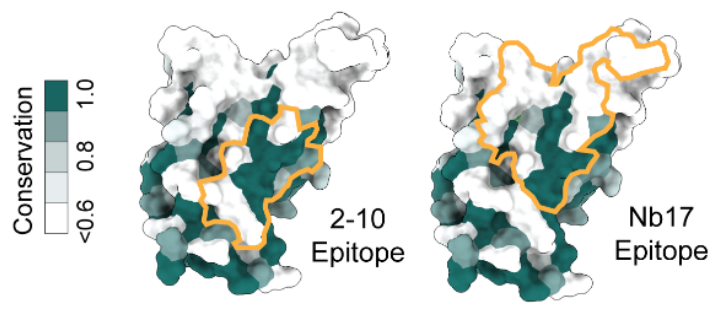

G

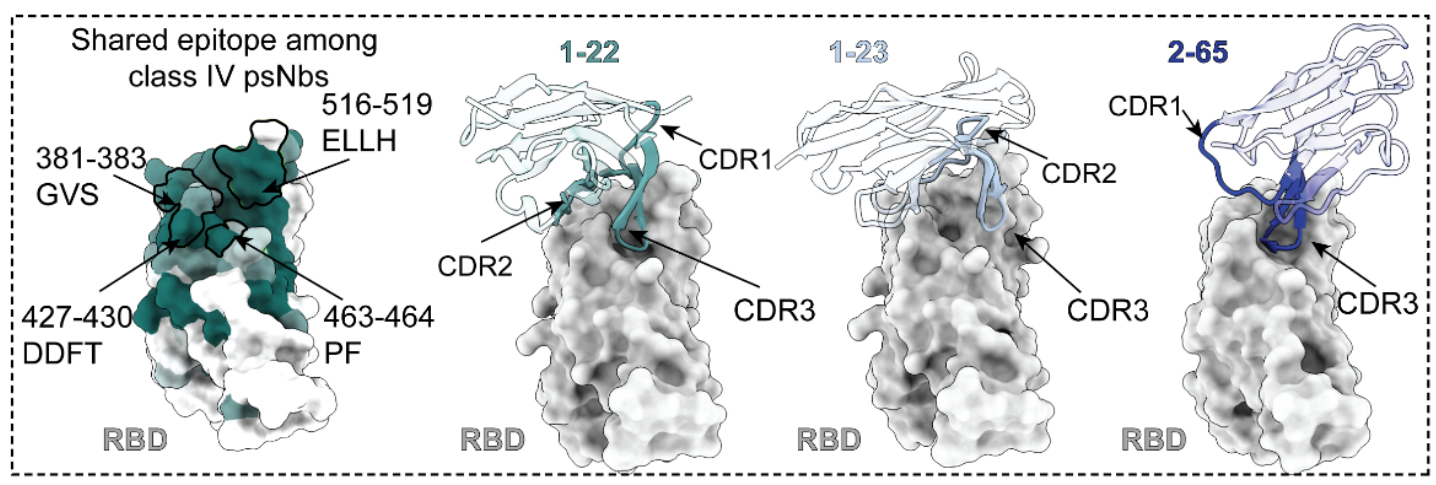

H
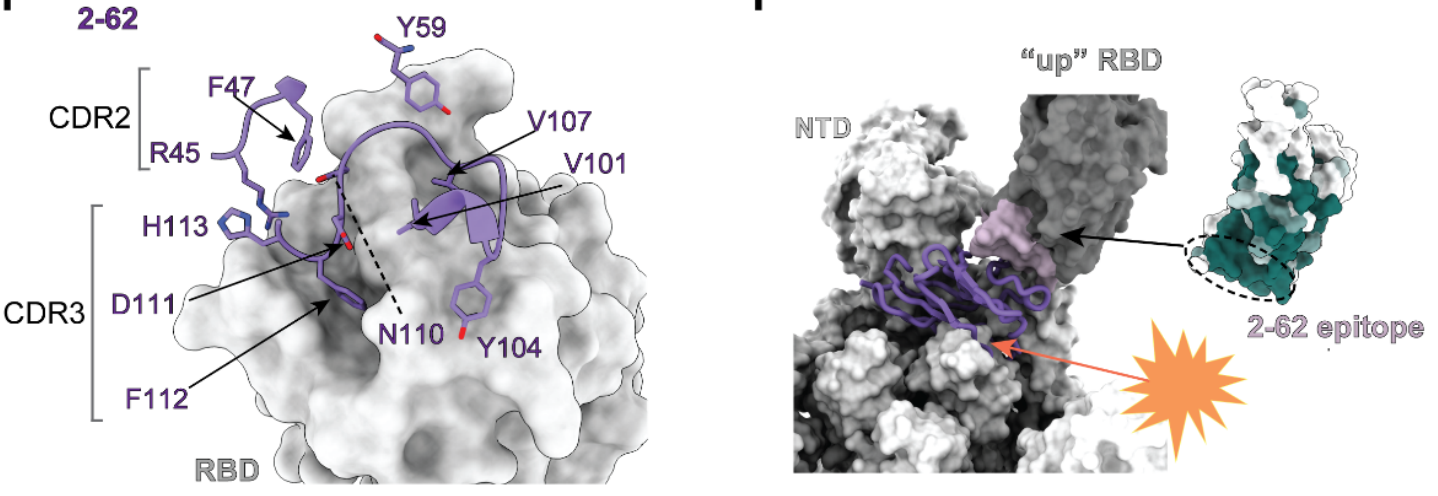
Fig. 5. Structural insights of four classes of psNbs. (A) Superposition of a class I psNb (2-67): RBD with hACE2 showing steric effects. (B) 2-67 epitope (green) and critical VOC mutations (red) on RBD. (C) Heatmap of relative binding energy contribution of $\mathrm{Nbs}$ per epitope residue that overlaps with critical mutations from VOCs. (D) Surface representation showing all CDRs of 2-67 with RBD. Green: 2-67; white: RBD. (E) Superposition of a Class III psNb (2-10) and a non-psNb (Nb17). (F) The conservation map of RBD and the epitopes of a Class III psNb (2-10) and Nb17. (G) Shared epitopes and binding of three class IV psNbs (1-22, 1-23 and 2-65) to the RBD. Shared epitope residues are highlighted on the RBD structure with sequences labeled on the side. $(\mathbf{H})$ Binding of a class V (2-62) with the RBD. Interacting CDR residues are labeled. (I) Superposition of 2-62 from the 2-62:RBD structure showing the clash between 2-62 and the spike in the allRBD-up conformation. NTD: N-terminal domain. Representation showing the 2-62 epitope is conserved but not accessible on the spike. 


\section{Figure 6}

A

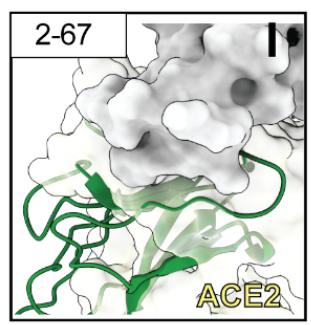

B

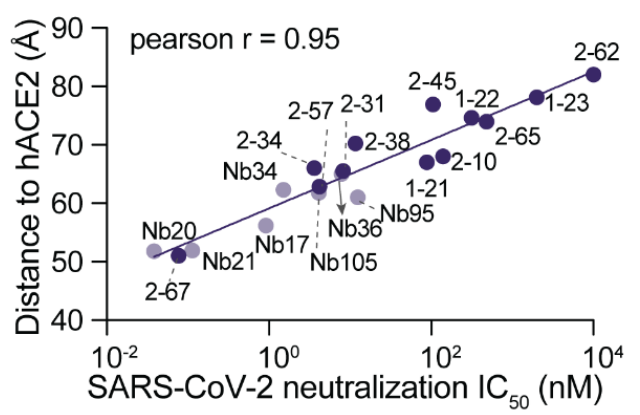

D
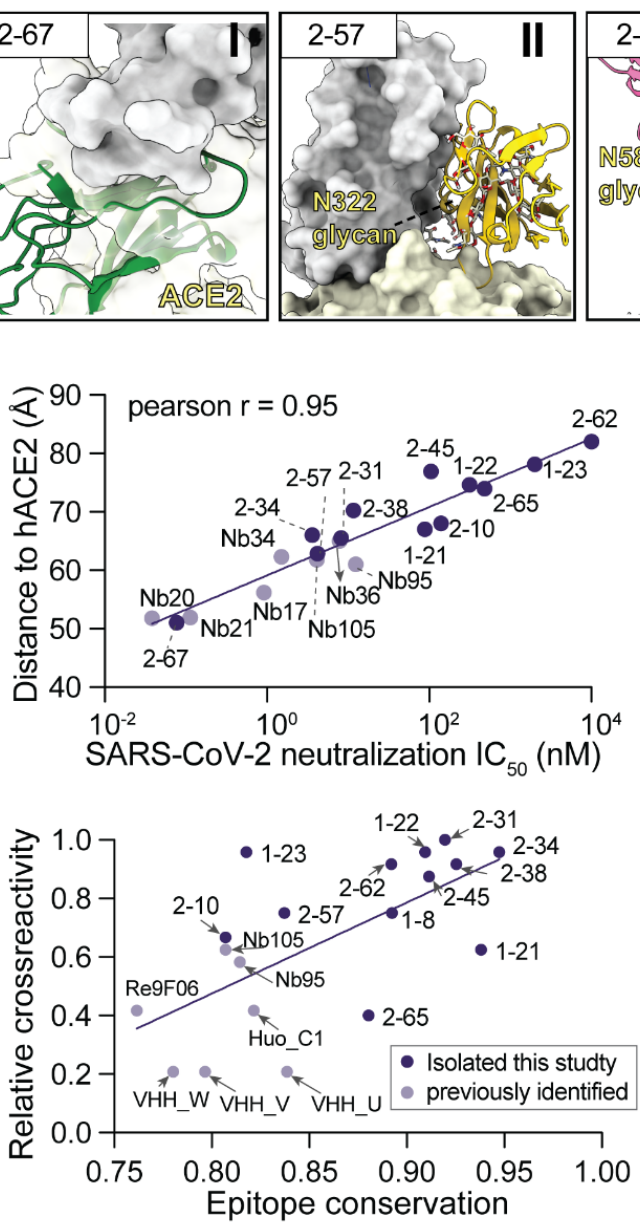
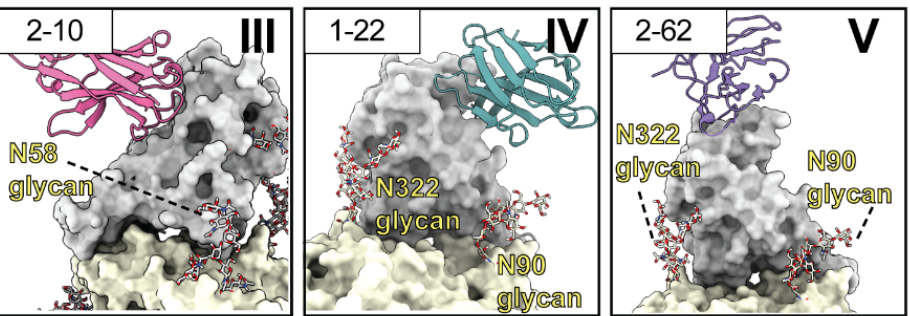

C

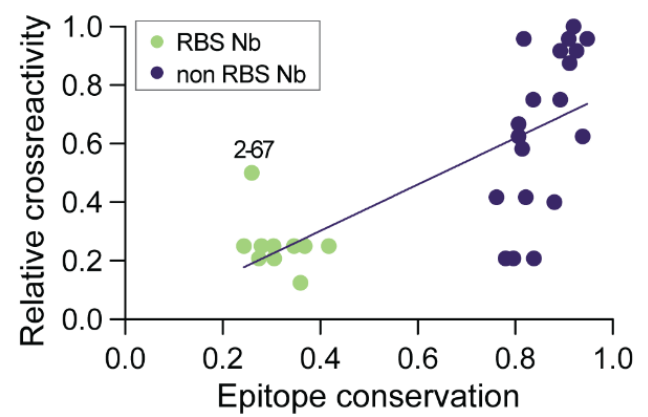

E

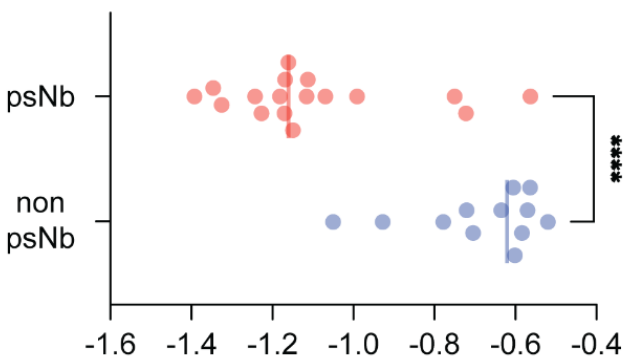

Average viral fitness score of epitope residues

$\mathbf{F}$

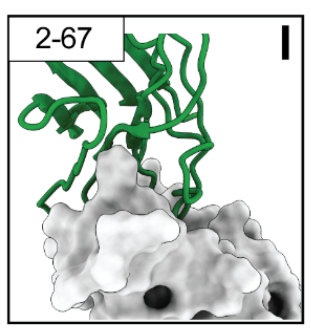

G

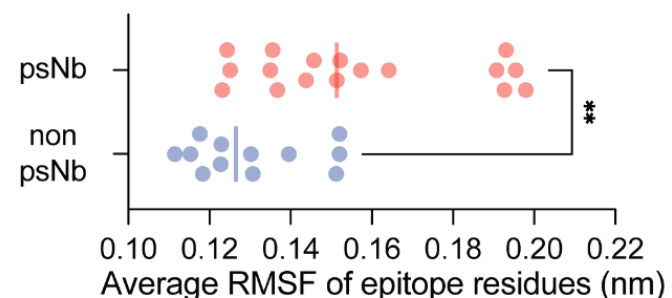

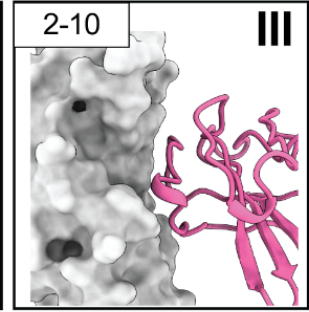

H

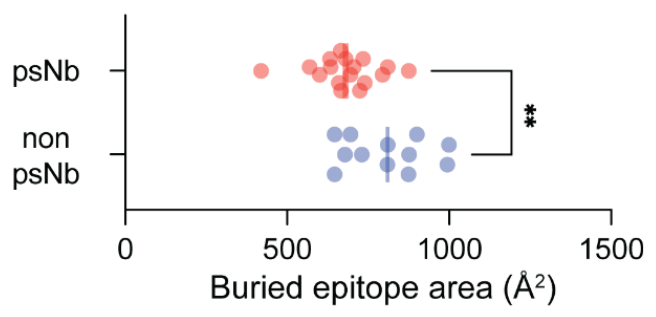


Fig. 6. Mechanisms of broad neutralization. (A) Superpositions of different psNb classes with RBD and hACE2. Key hACE2 glycosylation sites are shown (N90 and N322). (B) Correlation between neutralization potency and the distance between $\mathrm{Nb}$ epitope and RBS. The distance is calculated based on centroids of the $\mathrm{Nb}$ epitope and hACE2 epitope. (C-D). Correlation between cross-reactivity and epitope conservation for C) all RBD Nbs and d) non-RBS Nbs. The relative cross-reactivity is represented by the normalized ELISA binding of 4 sarbecovirus RBD clades (table S4). (E) Comparison of the averaged viral fitness score of RBD epitope residues between psNbs and non-psNbs. The fitness score (31) is obtained by evaluating the mutational effects on RBD expression level. Negative values correspond to higher loss of fitness. (F) Structures showing the geometric features of $\mathrm{Nb}: \mathrm{RBD}$ interfaces for 5 classes of psNbs. (G) Comparison of average root-meansquare-fluctuation (RMSF) of epitope residues between psNbs and non-psNbs (table S5). (H) Comparison of buried surface area of epitope between psNbs and non psNbs. 


\section{Supplementary Materials for}

\section{Super-immunity by broadly protective nanobodies to sarbecoviruses}

Yufei Xiang ${ }^{1 *}$, Wei Huang ${ }^{2 *}$, Hejun Liu ${ }^{3 *}$, Zhe Sang $^{4 *}$, Sham Nambulli5,6, Jérôme Tubiana ${ }^{7,8}$, Kevin L Williams Jr. $^{5,6}$, W Paul Duprex ${ }^{5,6}$, Dina Schneidman-Duhovny ${ }^{7}$, Ian A. Wilson ${ }^{3,9}$, Derek J. Taylor ${ }^{2,10}$, and Yi Shi ${ }^{1,4, \S}$

${ }^{1}$ Department of Cell Biology, University of Pittsburgh, Pittsburgh, PA, 15213, USA

${ }^{2}$ Department of Pharmacology, Case Western Reserve University, Cleveland, OH 44106, USA

${ }^{3}$ Department of Integrative Structural and Computational Biology, The Scripps Research Institute, La Jolla, CA, 92037, USA

${ }^{4}$ The University of Pittsburgh and Carnegie Mellon University Program for Computational Biology, Pittsburgh, PA, 15213, USA

${ }^{5}$ Center for Vaccine Research, University of Pittsburgh, Pittsburgh, PA, 15213, USA

${ }^{6}$ Department of Microbiology and Molecular Genetics, University of Pittsburgh, Pittsburgh, PA, 15213, USA

${ }^{7}$ School of Computer Science and Engineering, The Hebrew University of Jerusalem, Israel

${ }^{8}$ Blavatnik School of Computer Science, Tel Aviv University, Israel

${ }^{9}$ Skaggs Institute for Chemical Biology, The Scripps Research Institute, La Jolla, CA, 92037, USA

${ }^{10}$ Department of Biochemistry, Case Western Reserve University, Cleveland, OH 44106, USA

*These authors contributed equally to this work

§Correspondence: yi.shi@pitt.edu 


\section{Materials and Methods}

\section{Purification of recombinant sarbecovirus RBDs and SARS-CoV-2 spike}

The mammalian expression vectors (43) encoding the RBDs of RaTG13-CoV (GenBank QHR63300; S protein residues 319-541), SHC014-CoV (GenBank KC881005; residues 307-524), Rs4081-CoV (GenBank KY417143; residues 310-515), pangolin17-CoV (GenBank QIA48632; residues 317-539), RmYN02-CoV (GSAID EPI_ISL_412977; residues 298-503), Rf1-CoV (GenBank DQ412042; residues 310-515), W1V1-CoV (GenBank KF367457; residues 307-528), Yun11-CoV (GenBank JX993988; residues 310-515), BM-4831-CoV (GenBank NC014470; residues 310-530), BtkY72-CoV (GenBank KY352407; residues 309-530) with an Nterminal Mu phosphatase signal peptide and C-terminal His-tag were a kind gift from Pamela J. Bjorkman's lab, Caltech. Plasmids of the RBDs for the following sarbecovirus strains were synthesized from Synbio Technologies in a similar way: SARS-CoV-2 (GenBank MN985325.1; S protein residues 319-539), SARS-CoV (GenBank AAP13441.1; residues 318-510), Rs7327-CoV (GenBank KY417151.1; residues 319-518), Rs4092CoV (GenBank KY417145.1; residues 314-496), YN2013-CoV (GenBank KJ473816.1; residues 314-496), ZC45-CoV (GenBank MG772933.1; residues 327-509), HKU3-1-CoV (GenBank DQ022305.2; residues 322505), Shaanxi2011-CoV (GenBank JX993987.1; residues 321-502) and Rp3-CoV (GenBank DQ071615.1; residues 322-504). The cDNA encoding SARS-CoV-2 spike HexaPro (S) was obtained from Addgene (Cat\# 154754) (44). To express the proteins, Expi293F cells were transiently transfected with the plasmid using the ExpiFectamine 293 kit (Thermo, Cat\#A14635). After 24 hrs of transfection, enhancers were added to further boost protein expression. Cell culture was harvested 5-6 days after transfection and the supernatant was collected by high-speed centrifugation at $21,000 \times \mathrm{g}$ for $30 \mathrm{~min}$. The secreted proteins in the supernatant were purified using His-Cobalt resin (Thermo). Eluted proteins were then concentrated and further purified by sizeexclusion chromatography using a Superose 6 10/300 (for S) or Superdex 75 column (for RBDs, Cytiva) in a buffer composed of $20 \mathrm{mM}$ Hepes $\mathrm{pH} 7.5$ and $150 \mathrm{mM} \mathrm{NaCl}$. SARS-CoV-2 RBD variants were obtained from the Acro Biosystems.

\section{Successive camelid immunization with RBD and the proteomic identification of psNbs}


A llama ("Wally") was immunized with an RBD-Fc fusion protein (Acro Biosystems, Cat\#SPD-c5255) at a primary dose of $0.2 \mathrm{mg}$ (with complete Freund's adjuvant), followed by three consecutive boosts of $0.1 \mathrm{mg}$ every 2 weeks. The initial bleed was collected 10 days after the final boost as previously described (14). Three weeks after the collection of the initial bleed, the llama was immunized again by four consecutive boosts every 2 weeks. The booster bleed was collected 10 days after the final boost. The immunization procedures were performed by Capralogics, Inc. following the IACUC protocol.

To isolate $\mathrm{V}_{\mathrm{H}} \mathrm{H}$ antibodies, plasma was first purified from the immunized bleeds by the Ficoll gradient (Sigma). Polyclonal $\mathrm{V}_{\mathrm{H}} \mathrm{Hs}$ mixtures were then isolated from the plasma using a two-step purification protocol (45). RBDs

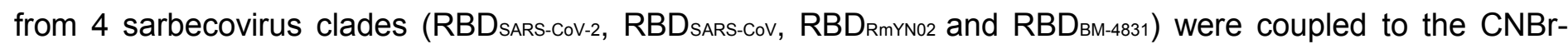
activated sepharose resin for affinity isolation of RBD-specific $V_{H} H s$. After binding, RBD-specific $V_{H} H s$ were eluted and proteolyzed as previously described $(14,15)$. Efficiently digested peptides were subjected to proteomics analysis by using the nano-LC 1200 that was coupled online with a $\mathrm{Q}$ Exactive ${ }^{\mathrm{TM}} \mathrm{HF}-\mathrm{X}$ Hybrid Quadrupole Orbitrap ${ }^{\mathrm{TM}}$ mass spectrometer. The MS data obtained from different RBD-specific $\mathrm{V}_{\mathrm{H}} \mathrm{H}$ isolations were analyzed by AugurLlama to identify high-affinity Nbs for each RBD (15). To facilitate the identification of psNbs, the abundance of $\mathrm{Nb}$ CDR3 peptides was quantified across different $\mathrm{V}_{\mathrm{H}} \mathrm{H}$ isolations. psNbs (represented by CDR3s) are assigned based on two criteria. 1) they must be classified as high-affinity binders

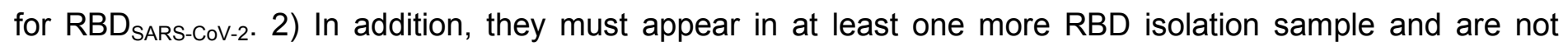
classified as low affinity binders (15).

\section{Nb DNA synthesis and cloning}

The monomeric Nb genes were codon-optimized and synthesized (Synbio). All the Nb DNA sequences were cloned into a pET-21b(+) vector using EcoRI and HindIII restriction sites. The monomeric Nbs 2-31 and 2-45 were also cloned into a $\mathrm{pET}-22 \mathrm{~b}(+)$ vector at the BamHI and Xhol sites for periplasmic expression. To produce a heterodimeric $\mathrm{Nb}$ 132-118, the DNA fragment of the monomeric $\mathrm{Nb} 2-31$ was first PCR amplified from the pET-21b(+) vector to introduce a linker sequence and two restriction sites of Xhol and HindIII that facilitate cloning:

Primer1: ccCAAGCTTggtggtggtggtagtggtggtggtggtagtggtggtggtggtagtCAaGTTCAACTGGTTGAATCTG; Primer2: ccgCTCGAGTGCGGCCGCcagtttGCTACTAACGGTAACTT. 
The PCR fragment was then inserted into the $2-45 \mathrm{pET}-21 \mathrm{~b}(+)$ vector at the same restriction sites to produce the heterodimer 2-45-(GGGGS) 3 -2-31.

\section{Purification of Nbs}

$\mathrm{Nb}$ DNA constructs were transformed into BL21(DE3) cells and plated on Agar with $50 \mu \mathrm{g} / \mathrm{ml}$ ampicillin at $37{ }^{\circ} \mathrm{C}$ overnight. Cells were cultured in an LB broth to reach an O.D. of $\sim 0.5-0.6$ before IPTG (0.5 - 1 mM) induction at $16 / 20^{\circ} \mathrm{C}$ overnight. Cells were then harvested, sonicated, and lysed on ice with a lysis buffer (1xPBS, $150 \mathrm{mM} \mathrm{NaCl}, 0.2 \%$ TX-100 with protease inhibitor). After cell lysis, protein extracts were collected by centrifugation at 21,000 x g for 10 mins and the his-tagged Nbs were purified by the Cobalt resin (Thermo) and natively eluted with a buffer containing $150 \mathrm{mM}$ imidazole buffer. Eluted $\mathrm{Nbs}$ were subsequently dialyzed in a dialysis buffer (e.g., 1x DPBS, pH 7.4 or SEC buffer).

For the periplasmic preparation of $\mathrm{Nbs}$ (2-31 and 2-45), cell pellets were resuspended in the TES buffer $(0.1 \mathrm{M}$ Tris- $\mathrm{HCl}, \mathrm{pH} 8.0 ; 0.25 \mathrm{mM}$ EDTA, $\mathrm{pH}$ 8.0; $0.25 \mathrm{M}$ Sucrose) and incubated on ice for 30 min. The supernatants were collected by centrifugation and subsequently dialyzed to DPBS. The resulting Nbs were then purified by Cobalt resin as described above.

\section{ELISA (enzyme-linked immunosorbent assay)}

Indirect ELISA was carried out to evaluate the camelid immune responses of the total single-chain only antibody $\left(\mathrm{V}_{\mathrm{H}} \mathrm{H}\right)$ to an $\mathrm{RBD}$ and to quantify the relative affinities of the psNbs. A 96-well ELISA plate (R\&D system) was coated with the RBD protein or the HEK-293T cell lysate at an amount of approximately 3-5 ng per well in a coating buffer (15 mM sodium carbonate, $35 \mathrm{mM}$ sodium bicarbonate, $\mathrm{pH} 9.6)$ overnight at $4^{\circ} \mathrm{C}$, with subsequent blockage with a blocking buffer (DPBS, v/v 0.05\% Tween 20,5\% milk) at room temperature for 2 hours. To test the immune response, the total $V_{H} H$ was serially 5 -fold diluted in the blocking buffer and then incubated with the RBD-coated wells at room temperature for 2 hours. HRP-conjugated secondary antibodies against llama Fc were diluted 1:75,00 in the blocking buffer and incubated with each well for an additional 1 hour at room temperature. For the initial screening of $\mathrm{Nb}$ binding against $4 \mathrm{RBDs}$, scramble $\mathrm{Nbs}$ that do not bind the RBDs were used as negative controls. Nbs were serially 10 -fold diluted from $1 \mu \mathrm{M}$ to $1 \mathrm{nM}$ in the blocking buffer. For the Nb affinity measurements against 24 RBDs, Nbs were serially 4-fold diluted. The dilutions were incubated for 2 hours at room temperature. HRP-conjugated secondary antibodies against the 
T7-tag were diluted at 1:5,000 or 1:75,00 in the blocking buffer and incubated for 1 hour at room temperature.

Three washes with 1x PBST (DPBS, v/v 0.05\% Tween 20) were carried out to remove nonspecific absorbances between each incubation. After the final wash, the samples were further incubated in the dark with freshly prepared w3,3',5,5'-Tetramethylbenzidine (TMB) substrate for 10 mins at room temperature to develop the signals. After the STOP solution (R\&D system), the plates were read at multiple wavelengths (450 $\mathrm{nm}$ and $550 \mathrm{~nm}$ ) on a plate reader (Multiskan GO, Thermo Fisher). The raw data were processed by Prism 9 (GraphPad) to fit into a 4PL curve and to calculate logI $\mathrm{C}_{50}$.

\section{Competitive ELISA with recombinant hACE2}

A 96-well plate was pre-coated with recombinant spike-6P at $2 \mu \mathrm{g} / \mathrm{ml}$ at $4^{\circ} \mathrm{C}$ overnight. Nbs were 5 -fold diluted (from $0.2 / 1 / 5 \mu \mathrm{M}$ to $12.8 / 64 / 320 \mathrm{pM}$ ) in the assay buffer with a final amount of $50 \mathrm{ng}$ biotinylated hACE2 at each concentration and then incubated with the plate at room temperature for $2 \mathrm{hrs}$. The plate was washed by the washing buffer to remove the unbound hACE2. 1:6000 diluted Pierce ${ }^{\mathrm{TM}}$ High Sensitivity NeutrAvidin ${ }^{\mathrm{TM}}-\mathrm{HRP}$ (Thermo fisher cat\# 31030) were incubated with the plate for $1 \mathrm{hr}$ at room temperature. TMB solution was added to react with the HRP conjugates for 10 mins. The reaction was then stopped by the Stop Solution. The signal corresponding to the amount of the bound hACE2 was measured by a plate reader at $450 \mathrm{~nm}$ and 550 $\mathrm{nm}$. The wells without Nbs were used as control to calculate the percentage of hACE2 signal. The resulting data were analyzed by Prism 9 (GraphPad) and plotted.

\section{psNb epitope analysis by competitive size exclusion chromatography (SEC)}

Analytical size exclusion chromatography was performed with a Superdex 75 increase GL column (column volume: $24 \mathrm{~mL}$, Cytiva) on a Shimadzu HPLC system equipped with a multi-wavelength UV detector at a flow rate of $0.3 \mathrm{~mL} / \mathrm{min}$. The column was connected and placed in a column oven set to $15^{\circ} \mathrm{C}$, and the SEC running buffer was $10 \mathrm{mM}$ HEPES $\mathrm{pH} 7.1,150 \mathrm{mM} \mathrm{NaCl}$. A reference SEC profile for RBD with epitope I benchmark $\mathrm{Nb}$ (Nb21), epitope II benchmark $\mathrm{Nb}$ (Nb105) and epitope III benchmark $\mathrm{Nb}$ (Nb36) was performed after column equilibration. Subsequently, three separate runs with RBD and specified psNb were performed after mixing with (i) benmark I and benchmark II, (ii) benchmark II and III and (iii) benchmark I and III Nbs. A supershift of the peak, at the same or to the left of the RBD and three Nbs peak in the reference profile, in run 
(i) but not run (ii) and (iii) sorts a psNb into group C. Similarly, psNb with a supershift in run (ii) but not run (i) and (iii) belongs to group A, and a supershift in run (iii) but not run (i) and (ii) belongs to group B. When supershifts were observed in all three runs, the psNb was sorted into a group $\mathrm{D}$. psNbs were sorted into group E if the supershifts were not detected on SEC.

\section{Nb affinity measurement by SPR}

Surface plasmon resonance (SPR, Biacore 3000 system, GE Healthcare) was used to measure Nb affinities. RBD proteins were_immobilized on the activated CM5 sensor-chip in $\mathrm{pH} 4.010 \mathrm{mM}$ sodium acetate buffer. The surface of the sensor chip was blocked by $1 \mathrm{M}$ Tris- $\mathrm{HCl}(\mathrm{pH}$ 8.5). For each $\mathrm{Nb}$ analyte, a series of concentration dilutions was injected in HBS-EP running buffer (GE-Healthcare), at a flow rate of $20 \mu \mathrm{l} / \mathrm{min}$ for $180 \mathrm{~s}$, followed by a dissociation time of 15 mins. Between each injection, the sensor chip surface was regenerated with the low $\mathrm{pH}$ buffer containing $10 \mathrm{mM}$ glycine- $\mathrm{HCl}(\mathrm{pH} 1.5-2.0)$. The regeneration was performed with a flow rate of $30-40 \mu \mathrm{l} / \mathrm{min}$ for $30-45 \mathrm{~s}$. The measurements were duplicated, and only highly reproducible data were used for analysis. Binding sensorgrams for each $\mathrm{Nb}$ were processed and analyzed using BIAevaluation by fitting with the 1:1 Langmuir model or the 1:1 Langmuir model with the drifting baseline.

\section{Pseudotyped SARS-CoV-2 neutralization assay}

The 293T-hsACE2 stable cell line (Cat\# C-HA101, Lot\# TA060720C) and pseudotyped SARS-CoV-2 (WuhanHu-1 strain, D614G, Alpha, Beta, Lambda, Delta and Omicron) particles with luciferase reporters were purchased from the Integral Molecular. The neutralization assay was carried out according to the manufacturers' protocols. In brief, 3- or 5-fold serially diluted $\mathrm{Nbs} /$ immunized $\mathrm{V}_{\mathrm{H}} \mathrm{H}$ mixture was incubated with the pseudotyped SARS-CoV-2-luciferase for accurate measurements. At least seven concentrations were tested for each $\mathrm{Nb}$ and at least two repeats of each $\mathrm{Nb}$ were done. Pseudovirus in culture media without $\mathrm{Nbs}$ was used as a negative control. $100 \mu \mathrm{l}$ of the mixtures were then incubated with $100 \mu \mathrm{l} 293 \mathrm{~T}$-hsACE2 cells at $2.5 \times 10 \mathrm{e}^{5} \mathrm{cells} / \mathrm{ml}$ in the 96 -well plates. The infection took $\sim 72 \mathrm{hrs}$ at $37^{\circ} \mathrm{C}$ with $5 \% \mathrm{CO}_{2}$. The luciferase signal was measured using the Renilla-Glo luciferase assay system (Promega, Cat\# E2720) with the luminometer at $1 \mathrm{~ms}$ integration time. The obtained relative luminescence signals (RLU) from the negative control wells were 
normalized and used to calculate the neutralization percentage at each concentration. Data were processed by

Prism 9 (GraphPad) to fit into a 4PL curve and to calculate the logI $C_{50}$ (half-maximal inhibitory concentration).

\section{SARS-CoV-2 Munich and Delta plaque reduction neutralization test (PRNT)}

Nbs were diluted in a 3- or 5-fold series in Opti-MEM (Thermo). Each Nb dilution (110 $\mu$ l) was mixed with $110 \mu \mathrm{l}$ of SARS-CoV-2 (Munich strain) containing 100 plaque-forming units (p.f.u.) or $110 \mu$ l of SARSCoV-2 (Delta strain, BEl Cat\# NR55611) containing 50 p.f.u. of the virus in Opti-MEM. The Nb-virus mixes $\left(220 \mu \mathrm{l}\right.$ total) were incubated at $37^{\circ} \mathrm{C}$ for $1 \mathrm{~h}$, after which they were added dropwise onto confluent Vero E6 cell (ATCC $®$ CRL-1586 ${ }^{\mathrm{TM}}$, for Munich) or Vero E6-TMPRSS2-T2A-ACE2 cells (BEI cat\# NR54970 , for Delta) monolayers in the six-well plates. After incubation at $37^{\circ} \mathrm{C}, 5 \%(\mathrm{v} / \mathrm{v}) \mathrm{CO}_{2}$ for $1 \mathrm{~h}, 2 \mathrm{ml}$ of $0.1 \%(\mathrm{w} / \mathrm{v})$ immunodiffusion agarose (MP Biomedicals) for Munich strain and $0.25 \% \quad(\mathrm{w} / \mathrm{v})$ immunodiffusion agarose for Delta strain in Dulbecco's modified eagle medium (DMEM) (Thermo) with $10 \%(v / v)$ FBS and $1 x$ pen-strep was added to each well. The cells were incubated at $37^{\circ} \mathrm{C}, 5 \% \mathrm{CO}_{2}$ for $72 \mathrm{hrs}$. The agarose overlay was removed and the cell monolayer was fixed with $1 \mathrm{ml} /$ well formaldehyde (Fisher) for $20 \mathrm{~min}$ at room temperature. The fixative was discarded and $1 \mathrm{ml} /$ well of $1 \%$ (w/v) crystal violet in $10 \%(\mathrm{v} / \mathrm{v})$ methanol was added. Plates were incubated at room temperature for 20 min and rinsed thoroughly with water. Plaques were then enumerated and the $50 \%$ plaque reduction neutralization titer $\left(\mathrm{PRNT}_{50}\right)$ was calculated. A validated SARS-CoV-2 antibody-negative human serum control and a validated NIBSC SARS-CoV-2 plasma control were obtained from the National Institute for Biological Standards and Control, UK) and an uninfected cells control were also used to ensure that virus neutralization by antibodies was specific.

\section{Biological safety}

All work with SARS-CoV-2 was conducted under biosafety level-3 (BSL-3) conditions in the University of Pittsburgh Center for Vaccine Research (CVR) and the Regional Biocontainment Laboratory (RBL). Respiratory protection for all personnel when handling infectious samples or working with animals was provided by powered air-purifying respirators (PAPRs; Versaflo TR-300; 3M, St. Paul, MN). Liquid and surface 
disinfection was performed using Peroxigard disinfectant (1:16 dilution), while solid wastes, caging, and animal wastes were steam-sterilized in an autoclave.

\section{Aerosolization of PiN using a soft-mist inhaler}

$\mathrm{Nb}$ was eluted and collected in the SEC running buffer (20 mM HEPES, $150 \mathrm{mM} \mathrm{NaCl}, \mathrm{pH} 7.5)$ and then concentrated to $0.5 \mathrm{ml}(1 \mathrm{mg} / \mathrm{ml})$. $\mathrm{Nb}$ was aerosolized by using a portable soft mist inhaler Pulmospray ${ }^{\circledR}$ (Resyca). Around $0.1-0.15 \mathrm{ml}$ dead volume was observed in the syringe and connector. The aerosols were collected in a $50 \mathrm{ml}$ falcon tube and SEC analysis was performed as described above.

\section{Cryo-electron microscopy data collection and image processing}

SARS-CoV-2 HexaPro spike at $1.1 \mathrm{mg} / \mathrm{mL}$ was incubated with 1.5 -fold molar excess of specified Nbs at room temperature for two hours. $3.5 \mu \mathrm{L}$ of 1 to 3 dilution sample using HBS buffer with $1 \%$ glycerol was applied onto a freshly glow discharged UltraAuFoil R1.2/1.3 grid (300 mesh) and plunge frozen using a vitrobot Mark IV (Thermo Fisher) with a blot force of 0 and $2.5 \mathrm{~s}$ blot time at $100 \%$ humidity at $4{ }^{\circ} \mathrm{C}$. The cryoEM datasets were collected at either CWRU or PNCC.

For CWRU datasets, movie stacks were recorded using an FEI Titan Krios transmission electron microscope G3i operated at $300 \mathrm{keV}$ and equipped with a Gatan K3 direct electron detector and Gatan BioQuantum image filter operated in zero-loss mode with a slit width of $20 \mathrm{eV}$. Automated data collection was carried out using serialEM (46) at a nominal magnification of $81,000 x$ with a physical pixel size of $1.07 \AA$ pixel (0.535 $\AA$ /pixel at super-resolution) for spike:nanobody complexes and $165,000 x$ with a physical pixel size of $0.52 \AA /$ pixel (0.26 Å/pixel at super-resolution) for RBD:nanobodies complexes. Each movie stack was collected with a dose rate of 18 electron/pixel/s in super-resolution mode and fractionated in 40 frames with two-second exposure, resulting in a total dose of $\sim 31.4 \mathrm{e} / \AA^{2}$ for $81,000 \mathrm{x}$ and in 32 frames with 1.5-second exposure, resulting in a total dose of $\sim 103.4 \mathrm{e} / \AA^{2}$ for $81,000 \mathrm{x}$. The number of movies for a specified dataset was listed in table S2. The defocus range was set to between -0.5 and $-2 \mu \mathrm{m}$.

For PNCC datasets, movie stacks were recorded using an FEl Titan Krios transmission electron microscope G3i operated at $300 \mathrm{keV}$ and equipped with a Gatan K3 direct electron detector and Gatan BioContinuum image filter operated in zero-loss mode with a slit width of $20 \mathrm{eV}$. Automated data collection was 
carried out using serialEM (46) at a nominal magnification of $64,000 x$ with a physical pixel size of $1.329 \AA /$ pixel (0.6645 $\AA$ /pixel at super-resolution). The dose rate was determined over a sample hole to calculate the exposure time resulting in a total dose of $40 \mathrm{e} / \AA^{2}$ and the exposures were fractionated into a total of 40 frames. The number of movies for a specified dataset was listed in table S2. The defocus range was set to between 0.5 and $-2 \mu \mathrm{m}$.

Image processing was performed on-the-fly using CryoSPARC Live version 3.2 (47-49). The particles were automatically picked using the blob picker with $240 \AA$ or $100 \AA$ diameter for spike:nanobody or RBD:nanobodies, respectively. Reference-free 2D classification was performed in streaming with 200 classes and limited maximum resolution to $18 \AA$. Upon the completion of data collection, the selected particles from the good 2D class averages were subjected for another round of 2D classification with 200 classes, and particles from classes with resolution better than $10 \AA$ and ECA less than 2 were selected for subsequent analysis. These 2D classes were submitted for a "rebalance 2D" job type to trim particles from dominant views. The rebalanced particle set was then used for ab-initio reconstruction to generate the initial volume. 3D refinement was first carried out using non-uniform refinement using ab-initio volume as the reference without mask. To resolve the density for nanobodies, an RBD structure (PDB 6M0J) (27) was docked into the cryoEM density and the structural model for nanobody was manually placed into the additional density accounted for nanobodies to ensure the correct orientation. The resulting RBD:nanobody model was used to generate the mask for focused 3D classification in CryoSPARC version 3.3.1 with six classes and target resolution of $6 \AA$, and PCA was chosen as the initialization mode with the parameter "number of components" set for 2 . 3D classes with both RBD and nanobody densities well resolved were selected for sequent new local refinement with the mask around RBD and nanobody. The gold-standard Fourier shell correlation (FSC) of 0.143 criterion was used to report the resolution and the FSC curves were corrected for the effects of a soft mask using highresolution noise substitution (50).

\section{Model building and refinement}

PDB entry, 7CAK (51), was used as the initial model for spike excluding RBD and the missing fragment for residues 621 to 640 was modeled de novo in Coot (52). RBD from PDB entry, 6M0J (27), was used as the initial model and nanobodies structures were generated using ColabFold (53). After assembling individual 
components into a single PDB file, the models were refined into the composite map using phenix.real_space_refine (54). The glycans were built using the carbohydrate module in Coot (55). Models were manually corrected in Coot (version 9.6.0) between rounds of read-space refinement in Phenix. All statistics for structural models were reported in table S2. Fig. panels depicting cryoEM maps or atomic models generated using ChimeraX (56). Maps colored by local resolution were generated using RELION 3.1 (57).

\section{Calculation for binding energy contribution}

Molecular dynamic (MD) simulations were used to generate short 1 ns trajectories for relative binding energy calculation. Input files for MD simulations of SARS-CoV-2 RBD and nanobody complexes (table S5) were prepared using tleap (58). MD simulations were performed using the NAMD (59) and the amber ff19sb (60), GLYCAM_06j (61), ions with the TIP3P water model (62). Proteins were solvated in a cubic water box with a $16 \AA$ padding in all directions. Sodium ions and chloride ions were added to achieve a physiological salt condition of $150 \mathrm{mM}$. The systems were energy minimized for 10,000 steps to remove bad contacts. Then, the systems were equilibrated with all heavy atoms restrained harmonically and the temperature raised $10 \mathrm{~K}$ per 10,000 steps starting from 0 to $300 \mathrm{~K}$ using temperature reassignment. After reaching the desired temperature, harmonic restraints were gradually reduced using a scale from 1.0 to 0 with a 0.2 decrements for every 50,000 steps. MD simulations were performed under the NPT ensemble $(63,64)$. Langevin dynamics was used for constant temperature control, with the value of Langevin coupling coefficient and the Langevin temperature set to $5 \mathrm{ps}$ and $300 \mathrm{~K}$, respectively. The pressure was maintained at $1 \mathrm{~atm}$ using the Langevin piston method with a period of $100 \mathrm{fs}$ and decay times of $50 \mathrm{fs}$. A time step of $2 \mathrm{fs}$ was used for all the simulations by using the SHAKE algorithm (65) to constrain bonds involving hydrogen atoms.

For each snapshot, every 10 ps of a $1 \mathrm{~ns}$ trajectory of SARS-CoV-2 RBD and nanobody complexes, the binding energy of MM/PBSA was calculated using Eqs. (1) and (2) $(66,67)$

$$
\begin{array}{ll}
\Delta G \quad \text { binding }=G \quad \text { complex }-G \quad R B D-G \quad N b(1) \\
\quad=\Delta E \quad M M+\Delta G \quad G B+\Delta G \text { nonpolar }-T \Delta S(2)
\end{array}
$$

where is the molecular mechanic (MM) interaction energy calculated in gas-phase between RBD and nanobody, including electrostatic and van der Waals energies; the desolvation free energy consists of polar 
(and nonpolar) terms; is the change of conformational entropy on nanobody binding, which was not considered here as the binding epitope on the RBD is very stable and the comparison was performed internally. The decomposition of the binding free energy to the relative energy contribution from individual residues was performed using the MMPBSA.py module in AMBER18 (68). The relative contribution to the binding energy from mutated residues observed in VOC were plotted as heatmap.

\section{Crystallographic analysis of psNbs with RBD}

The receptor-binding domain (RBD) of the SARS-CoV-2 spike (S) protein (GenBank: QHD43416.1), used in the crystallographic study, was cloned into a customized pFastBac vector (69), and fused with an N-terminal gp67 signal peptide and C-terminal $\mathrm{His}_{6}$ tag (70). Recombinant bacmids encoding each RBDs were generated using the Bac-to-Bac system (Thermo Fisher Scientific) followed by transfection into Sf9 cells using FuGENE HD (Promega) to produce baculoviruses for RBD expression. RBD protein was expressed in High Five cells (Thermo Fisher Scientific) with suspension culture shaking at 110 r.p.m. at $28{ }^{\circ} \mathrm{C}$ for 72 hours after the baculovirus transduction at an $\mathrm{MOI}$ of 5 to 10 . Supernatant containing RBD protein was then concentrated using a $10 \mathrm{kDa}$ MW cutoff Centramate cassette (Pall Corporation) followed by affinity chromatography using Ni-NTA resin (QIAGEN) and size exclusion chromatography using a HiLoad Superdex 200 pg column (Cytiva). The purified protein sample was buffer exchanged into $20 \mathrm{mM}$ Tris- $\mathrm{HCl} \mathrm{pH} 7.4$ and $150 \mathrm{mM} \mathrm{NaCl}$ and concentrated for binding analysis and crystallographic studies.

1-21+SARS-CoV-2 RBD and 2-31+SARS-CoV-2 RBD+CC12.1 complex were formed by mixing each of the protein components in an equimolar ratio and incubating overnight at $4^{\circ} \mathrm{C} .384$ conditions of the JCSG Core Suite (Qiagen) were used for setting-up trays for screening the 1-21 complex (12 mg/ml) and 2-31 complex $(14.3 \mathrm{mg} / \mathrm{ml})$ on our robotic CrystalMation system (Rigaku) at Scripps Research. Crystallization trials were setup by the vapor diffusion method in sitting drops containing $0.1 \mu \mathrm{l}$ of protein complex and $0.1 \mu \mathrm{l}$ of reservoir solution. Crystals appeared on day 3, were harvested on day 12, pre-equilibrated in cryoprotectant containing $0-10 \%$ ethylene glycol, and then flash cooled and stored in liquid nitrogen until data collection. X-ray diffraction data were collected at cryogenic temperature $(100 \mathrm{~K})$ at beamlines $23-\mathrm{ID}-\mathrm{D}$ of the Advanced Photon Source (APS) at Argonne National Laboratory and were collected from crystals grown in drops containing $20 \%$ 
polyethylene glycol $8000,0.1 \mathrm{M} \mathrm{NaCl}, 0.1 \mathrm{M} \mathrm{CAPS} \mathrm{pH} 10.5$ for the $1-21$ complex and drops containing $40 \%$ MPD, $0.1 \mathrm{M}$ cacodylate $\mathrm{pH} 6.5,5 \%(\mathrm{w} / \mathrm{v})$ polyethylene glycol 8000 for the 2-31 complex. Collected data were processed with HKL2000 (71). X-ray structures were solved by molecular replacement (MR) using PHASER (72) with original MR models for the RBD and Nanobody from PDB 7JMW (73) and PDB 7KN5 (19). Iterative model building and refinement were carried out in COOT (74) and PHENIX (75), respectively.

\section{RBD epitope analysis by ScanNet}

The epitope propensity profile of SARS-CoV-2 RBD (PDB 7jvb) was computed by ScanNet, a state-of-the-art geometric deep learning model for structure-based protein binding site prediction (76). We used the B-cell epitope network (ScanNet-BCE) without evolutionary information that was not trained on any SARS-CoV-1/2 antibody/antigen complex.

\section{Conservation and ScanNet analysis of viral antigens}

Dataset preparation. We collected all viral antibody - antigen complexes in PDB, as listed by SabDab (77) (release: 10/19/2021). Antigens were clustered at $70 \%$ sequence identity with a minimum length coverage of 15\% using CD-HIT (78), the Bio.align pairwise sequence alignment module and CATH domain identifiers. Briefly, we found that (i) ngram-based clustering using CD-HIT with default parameters overestimated the number of clusters, while (ii) computing all the entries of the pairwise sequence similarity matrix was intractable for our set of several thousands structures. We instead proceeded as follows: for a given sequence identity cut-off T (e.g. 100), sequences were clustered with CD-HIT, and the resulting representatives were further clustered at T-20. For each pair of T-representatives with identical CATH identifier or same T-20 cluster, the sequence identity and coverage were evaluated by pairwise alignment and the corresponding T-clusters were merged if necessary. The process was iterated at $\mathrm{T}=100 \%, 95 \%, 90 \%, 70 \%$ sequence identity cut-offs and yielded satisfactory clusters. We further grouped together the following antigen clusters that had lower sequence identity but high structure similarity: (i) Influenza hemagglutinin from strains H1N1, H3N2, H5N1, H7N9, H2N2 and (ii) Envelope protein of Dengue 1, Dengue 2, Dengue 4 and Zika. Only clusters with at least 7 unique antibodies were retained for further analysis, yielding 11 viral antigens. 
Antibody hit rate calculation. We constructed a multiple sequence alignment for each antigen cluster using MAFFT (79) and selected a representative structure with highest structure coverage and resolution. For each column of the alignment, the antibody hit rate was calculated as the fraction of unique antibodies binding it. For each complex, we identified the epitope residues as the antigen residues having at least one heavy atom within $4 \AA$ of at least one antibody heavy atom. Our analysis was performed on the antigen surface residues (relative accessible surface area $>=0.25$, computed within the biological assembly for multimeric antigens using Bio.DSSP). Finally, the column-wise antibody hit rate was projected back onto the surface residues representative structure.

Calculation of antigen conservation and epitope propensity. For each representative antigen chain, a multiple sequence alignment was constructed by homology search on the UniRef30_2020_06 sequence database using HHBlits (80) (4 iterations, default parameters). The alignment was deduplicated, hits with high gap content (>=25\% of the alignment) were discarded and the $10 \mathrm{~K}$ best hits were retained based on sequence identity. Each sequence was assigned a weight inversely proportional to the number of similar sequences found in the alignment $\left(90 \%\right.$ sequence identity cut-off). The amino acid frequency at each site $f_{i}(a)$ was subsequently calculated and the residue-wise conservation score was defined as $C_{i}=\ln (20)-S\left(f_{i}\right)$ where $S\left(f_{i}\right)=\sum_{a} f_{i}(a) \ln \left(f_{i}(a)\right)$. Conservation scores range from 0 to $\ln (20)=2.99$, higher is more conserved. We checked that this protocol correlated well with the ConSurf method based on phylogenetic trees (81). Epitope propensity scores were calculated with ScanNet-BCE.

\section{RMSF calculation}

Root mean square fluctuation of each RBD residue was calculated based on 100 ns Molecular Dynamics simulation trajectory. The simulation was run starting from the RBD structure (PDB 6lzg) using Gromacs 2020 version with the CHARMM36m force field (82). The RBD structure was solvated in transferable intermolecular potential with 3 points (TIP3P) water molecules and ions were added to equalize the total system charge. The steepest descent algorithm was used for initial energy minimization until the system converged at Fmax < $1,000 \mathrm{~kJ} /(\mathrm{mol} \cdot \mathrm{nm})$. Then water and ions were allowed to equilibrate around the protein in a two-step equilibration process. The first part of equilibration was at a constant number of particles, volume, and 
temperature (NVT). The second part of equilibration was at a constant number of particles, pressure, and temperature (NPT). For both MD equilibration parts, positional restraints of $\mathrm{k}=1,000 \mathrm{~kJ} /(\mathrm{mol} \cdot \mathrm{nm} 2)$ were applied to heavy atoms of the protein, and the system was allowed to equilibrate at a reference temperature of $300 \mathrm{~K}$, or reference pressure of 1 bar for 100 ps at a time step of $2 \mathrm{fs}$. Altogether 10,000 frames were saved for the RMSF analysis at intervals of 10 ps. To estimate average epitope RMSF, we defined epitope residues as residues with at least one atom within $4 \AA$ from the $\mathrm{Nb}$ atom and averaged RMSF over epitope residues.

\section{Phylogenetic analysis}

The phylogenetic analysis was performed to 1) 19 Sarbecovirus RBD sequences (Fig. 1a) 2) 100 psNb CDR3 sequences (fig. S2a) and 3) 32 SEC group B psNb and 3 previously characterized benchmark Nbs (Nb34, Nb95 and Nb105) CDR3 sequences (Fig. 4a). Sequences were aligned by MUSCLE (83) with default parameters. The phylogenetic tree was then constructed by the MEGA (84) using the maximized likelihood estimation method.

\section{Sarbecovirus RBD conservation}

To calculate Sarbecovirus RBD conservation with respect to SARS-CoV-2, 18 Sarbecovirus RBD sequences mentioned above were aligned to SARS-CoV-2 RBD sequence. After alignment, each SARS-CoV-2 RBD residue was compared to corresponding residue in other RBD sequences. An identical residue to that in SARS-CoV-2 is considered a match. The conservation for each RBD residue is calculated by the number of matches over 18.

\section{psNb epitope heat map}

An RBD residue and an $\mathrm{Nb}$ residue were defined in contact if the distance between any pair of their atoms was lower than a threshold of $4 \AA$. The $\mathrm{Nb}$ contact value of each RBD residue is calculated as the sum of all the $\mathrm{Nb}$ contacts.

\section{Measurement of buried surface area (BSA)}

The solvent-accessible surface area (SASA) of molecules was calculated by FreeSASA(85). The buried surface area in the case of the Nb-RBD complex was then calculated by equation:

$B S A=1 / 2[S A S A(N b)+S A S A(R B D)-S A S A($ complex $)]$

\section{Distance between $\mathrm{Nb}$ epitope and receptor binding sites}

The $\mathrm{Nb}$ epitope residues were extracted based on the method described above. The centroid of the Nb epitope 
residues is defined by the center of mass of atoms. Similarly, the centroid of receptor binding sites(RBS) was extracted. The distance between $\mathrm{Nb}$ epitope and RBS was calculated based on the distance between two centroids.

\section{Viral fitness score of epitope residues}

The viral fitness score was obtained from (31) by evaluating the mutational effects on RBD expression level. The fitness score of each RBD residue is the averaged value of mutating to any other amino acids. The viral fitness score of epitopes was calculated by averaging fitness score over epitope residues.

\section{Glycosylation modeling}

Based on the crystal structure of complex ACE2 and SARS-CoV-2 RBD contain seven (N53, N90, N103, N322, N432, N546, N690) and one (N343) N-glycosylation sites, respectively. A heterogeneous glycosylated system was set up for the complex based on previous study (29). The glycosylated ACE2:RBD complex was modeled by the Glycan Modeler module of the CHARMM-GUI webserver (86). 
A

\section{Figure S1}

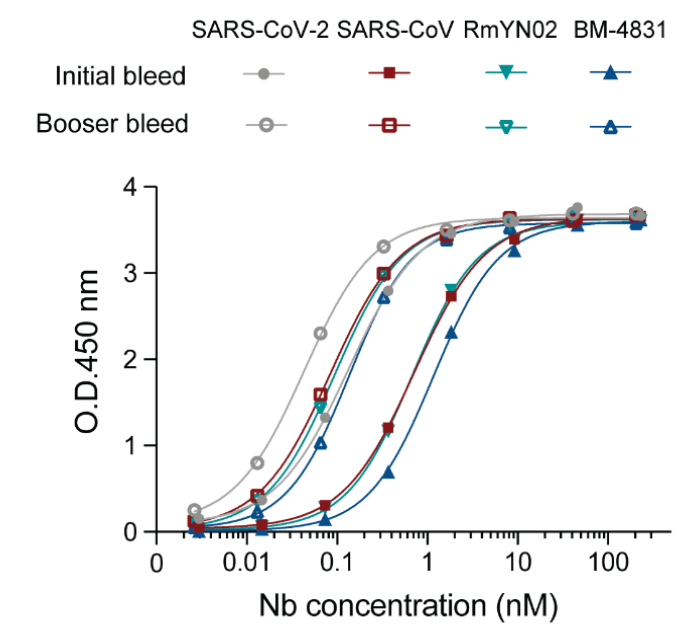

\begin{tabular}{c|c|c}
\multicolumn{3}{c}{ ELISA affinity $\mathrm{IC}_{50}(\mathrm{pM})$} \\
\hline SARS-CoV-2 RBD & 133 & 43 \\
\hline SARS-CoV RBD & 710 & 84 \\
\hline RmYN02-CoV RBD & 677 & 94 \\
\hline BM-4831-CoV RBD & 1169 & 136
\end{tabular}

B

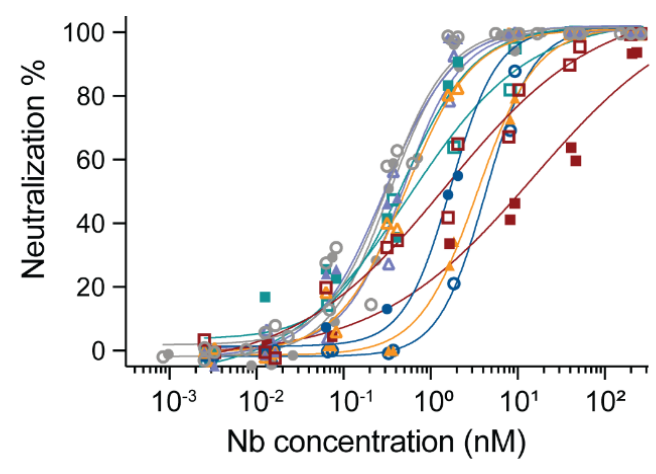

\begin{tabular}{|c|c|c|}
\hline \multicolumn{3}{|c|}{ Pseudovirus neutralization $\mathrm{IC}_{50}(\mathrm{pM})$} \\
\hline & Initial & Booster \\
\hline SARS-CoV-2 (D614G) & $\rightarrow 334$ & -0362 \\
\hline SARS-CoV & -15302 & - 1653 \\
\hline SARS-CoV-2 (Alpha) & $\triangle \quad 482$ & -331 \\
\hline SARS-CoV-2 (Beta) & -3478 & $-\triangle 577$ \\
\hline SARS-CoV-2 (Lambda) & - 552 & --542 \\
\hline SARS-CoV-2 (Delta) & -04310 & $\bullet-1901$ \\
\hline
\end{tabular}

Fig. S1. Analysis of the total $\mathrm{V}_{\mathrm{H}} \mathrm{Hs}$ isolated from serum after RBD immunization. (A) ELISA binding of total $\mathrm{V}_{\mathrm{H}} \mathrm{Hs}$ from the initial and booster bleeds against four representative RBDs. (B) Pseudovirus neutralization assay of total $\mathrm{V}_{\mathrm{H}} \mathrm{Hs}$ of the initial and booster bleeds against SARS-CoV-2, SARS-CoV-2 variants and SARSCoV. 


\section{Figure S2}

A

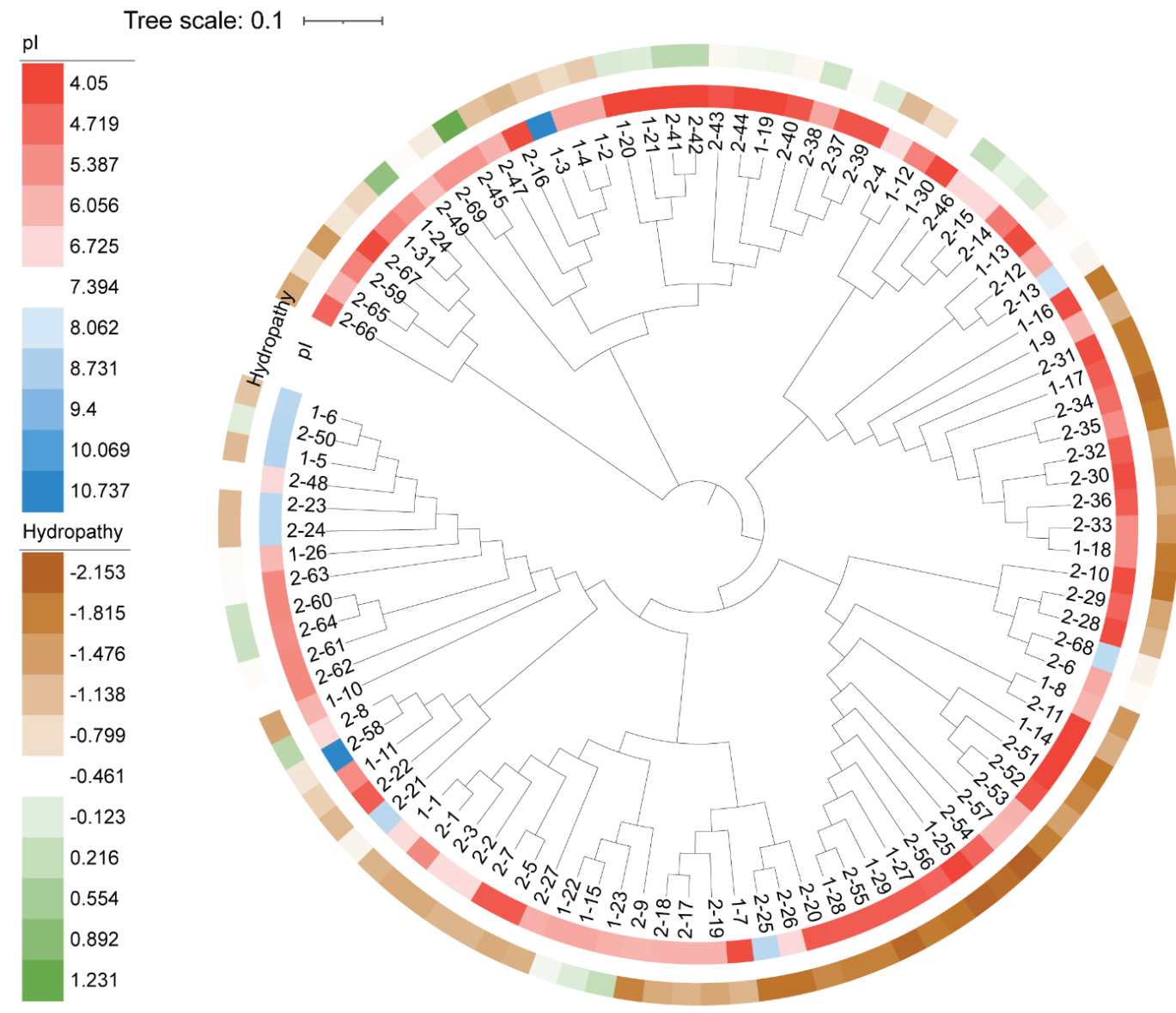

B

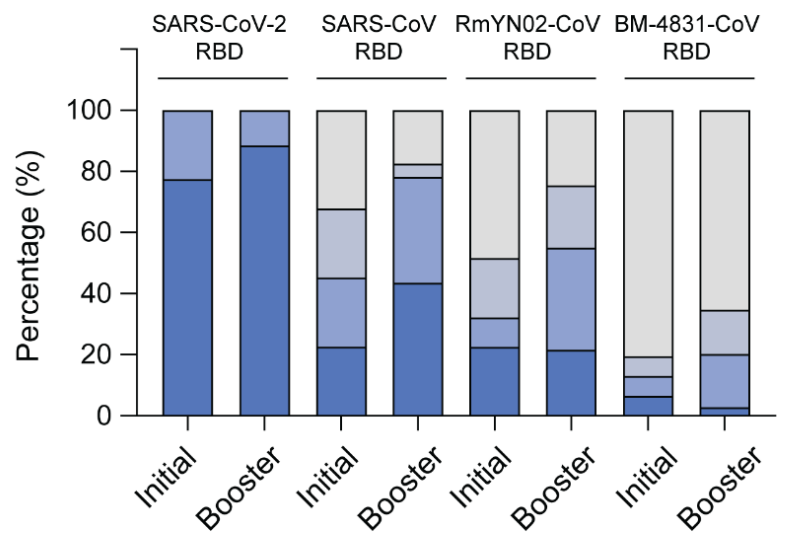

psNb ELISA

affinity range $(\mathrm{nM})$

$\square>1000$

$\square$ 100-1000

$\square$ 10-100

$\square<10$

Fig. S2. Analysis of experimentally verified psNbs. (A) Phylogenetic analysis of the psNb CDR sequences and their physicochemical properties including isoelectric point ( $\mathrm{pl}$ ), hydropathy. (B) The individual psNb isolated from either initial or booster bleed was synthesized, purified and evaluated for RBD sARs-Cov-2, RBDsARs-

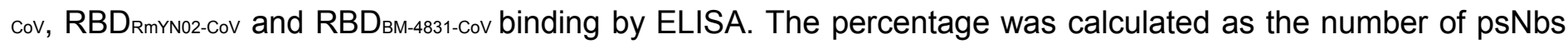
in a certain affinity range divided by the total number of the psNbs. 
A

\section{Figure S3}

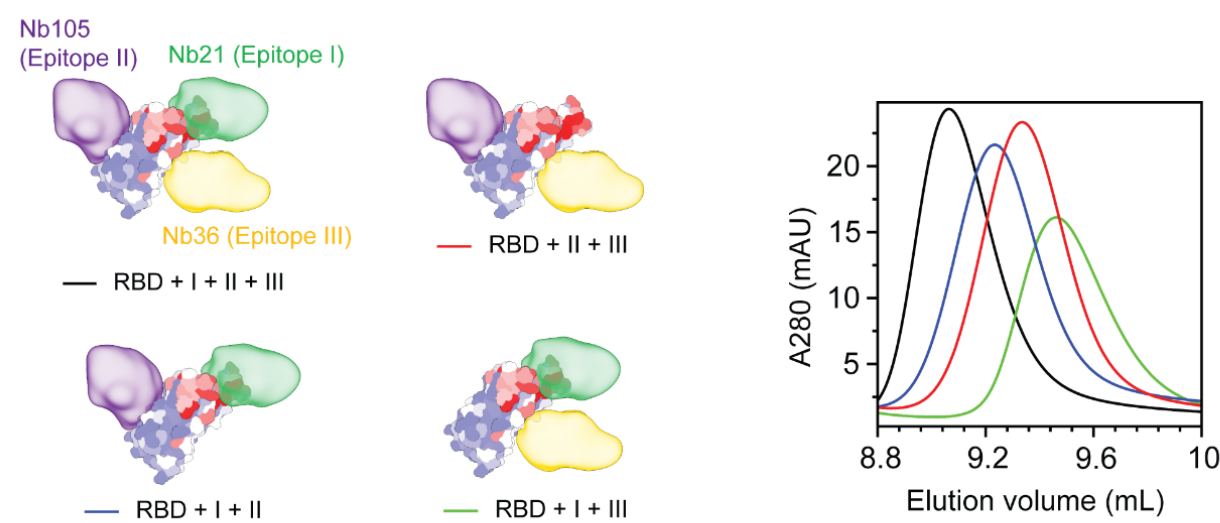

B
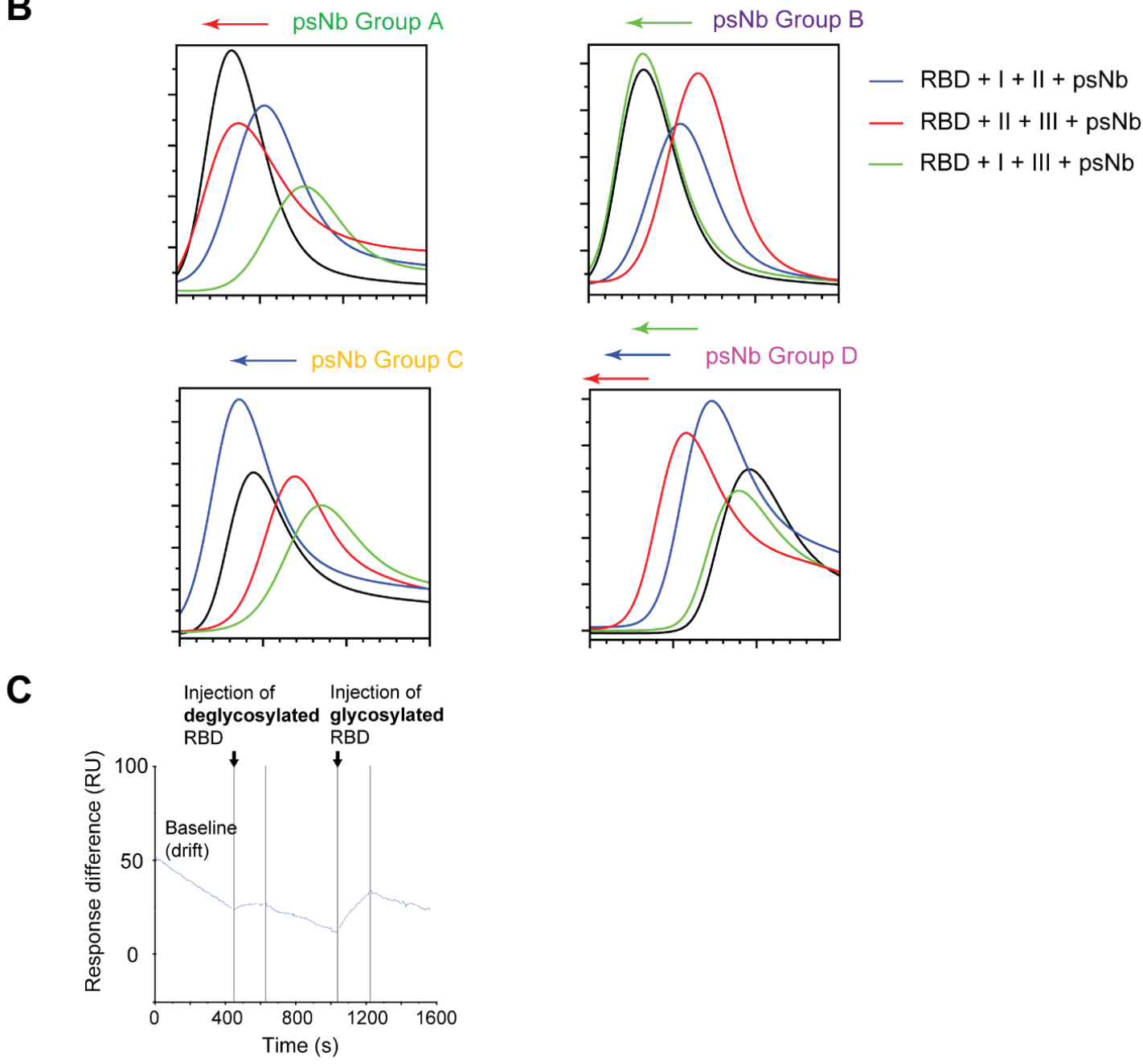

Fig. S3. Size exclusion chromatography (SEC) and surface plasmon resonance analysis of representative psNbs for RBD binding (summarized in table S1). (A) SEC profiles of the reconstituted nanobodies of non-overlapping epitopes in complex with $\mathrm{RBD}_{\mathrm{SARS}-\mathrm{CoV}-2}$. Left panel shows the structures that help illustrate competitive SEC experiments. (B) Competitive SEC of psNbs. Group A psNbs compete with Nb21. Group B psNbs compete with Nb105. Group C psNbs compete with Nb36. Group D psNbs do not 
bioRxiv preprint doi: https://doi.org/10.1101/2021.12.26.474192; this version posted December 28,2021 . The copyright holder for this preprint (which was not certified by peer review) is the author/funder, who has granted bioRxiv a license to display the preprint in perpetuity. It is made available under aCC-BY-NC-ND 4.0 International license.

compete with any of these three Nbs. Group E psNbs dissociate from RBD (Methods). (C) A representative SEC unclassified psNb (2-47) was covalently coated to the flow cell (Fc) 2 of a CM5 sensorchip. Fc1 of the sensorchip was non-coated as control. $1 \mu \mathrm{M}$ deglycosylated RBDsARs-Cov-2 in the HBS-EP buffer was injected to the surface at $20 \mu \mathrm{l} / \mathrm{min}$ for 3 mins with dissociation for 3 mins, followed by a sequential injection of $1 \mu \mathrm{M}$ glycosylated RBDsars-Cov-2. The responses were recorded as the difference between the signals from Fc2 and Fc1. 
bioRxiv preprint doi: https://doi.org/10.1101/2021.12.26.474192; this version posted December 28, 2021. The copyright holder for this preprint (which was not certified by peer review) is the author/funder, who has granted bioRxiv a license to display the preprint in perpetuity. It is made available under aCC-BY-NC-ND 4.0 International license.

\section{Figure S4}
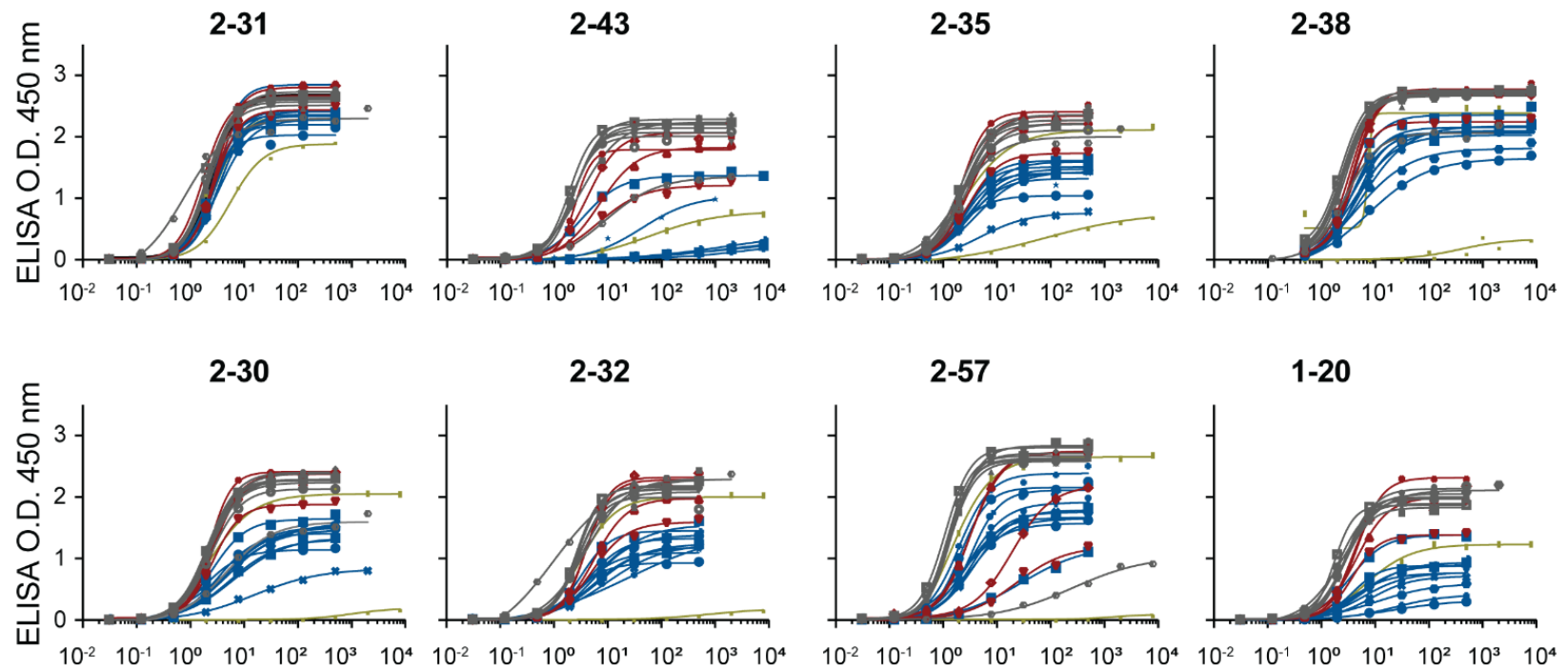

2-32

2-57

$1-20$

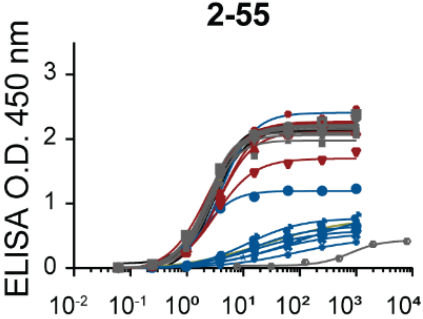

1-22
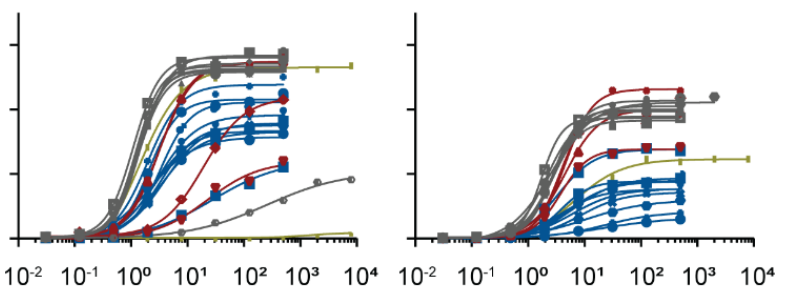

$\mathrm{Nb}$ concentration $(\mathrm{nM})$

2-10
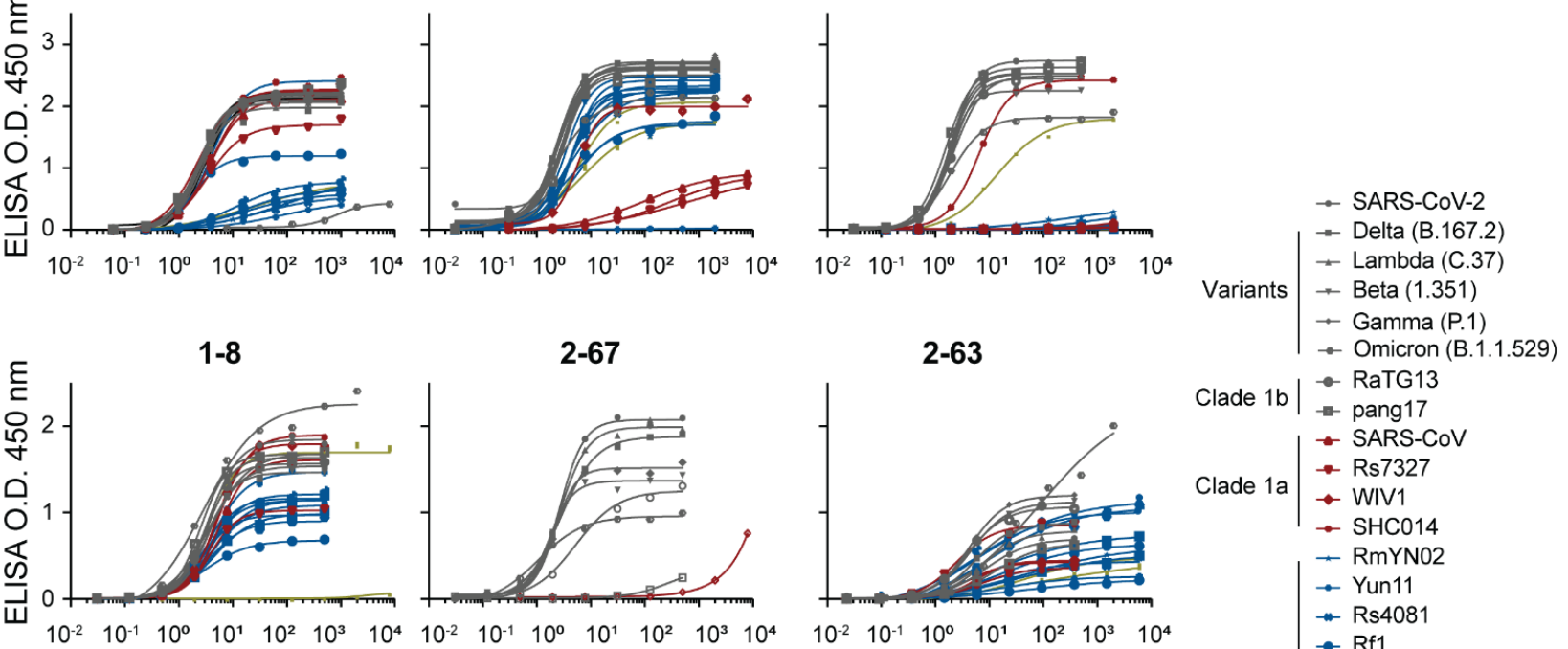

2-67

2-63
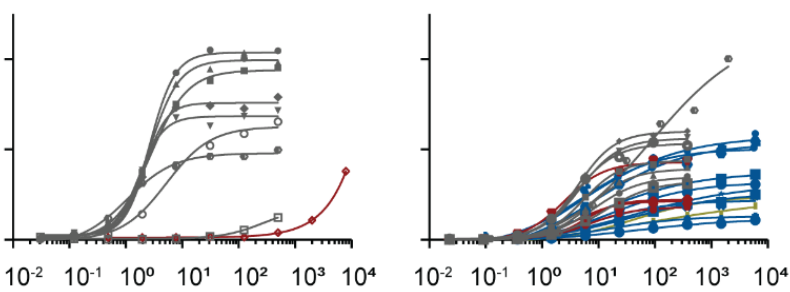

Clade $1 \mathrm{~b}$

- SARS-CoV

Clade 1a $\rightarrow$ Rs7327

$\begin{aligned} & \rightarrow \text { WIV1 } \\ & \rightarrow \text { SHC014 }\end{aligned}$

- RmYN02

$\rightarrow$ Yun11

* Rs4081

$\rightarrow$ Rf1

Clade 2

- Rf4092

- YN2013

- ZC45

- HKU3-1

- Shaanxi2011

$\rightarrow$ Rp3

Clade 3| \begin{tabular}{l|l}
$-\mathrm{BM}-4831$ \\
$-\mathrm{BtKY72}$
\end{tabular}

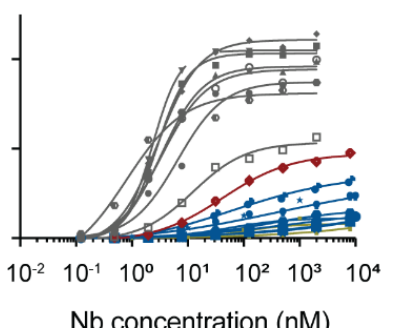

$\mathrm{Nb}$ concentration $(\mathrm{nM})$

$\mathrm{Nb}$ concentration $(\mathrm{nM})$

$\mathrm{Nb}$ concentration $(\mathrm{nM})$

Fig. S4. ELISA of 17 psNbs against the RBDs from the WT SARS-CoV-2, variants and 18 different sarbecoviruses that span all four clades. 
bioRxiv preprint doi: https://doi.org/10.1101/2021.12.26.474192; this version posted December 28, 2021. The copyright holder for this preprint (which was not certified by peer review) is the author/funder, who has granted bioRxiv a license to display the preprint in perpetuity. It is made available under aCC-BY-NC-ND 4.0 International license.

\section{Figure S5}

2-31

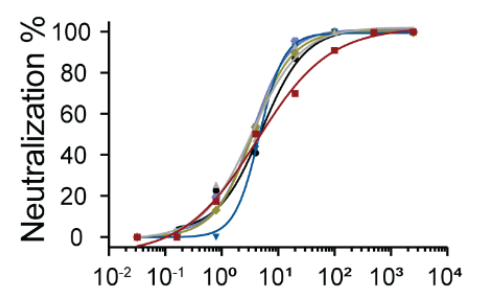

2-30

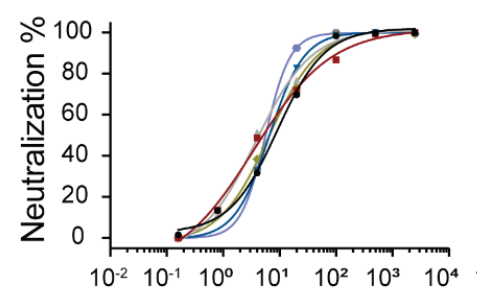

1-20

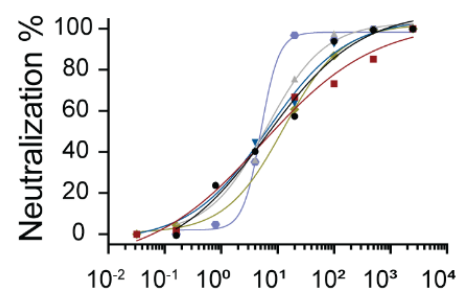

2-10

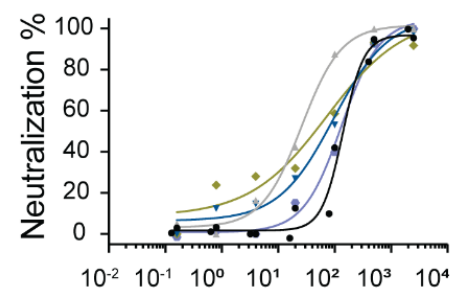

$\mathrm{Nb}$ concentration $(\mathrm{nM})$
2-34

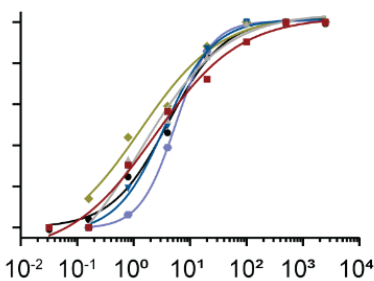

2-35

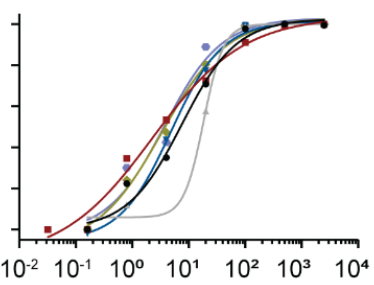

2-38

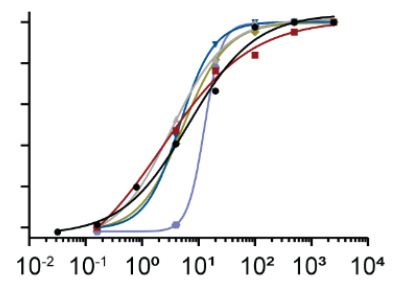

2-57
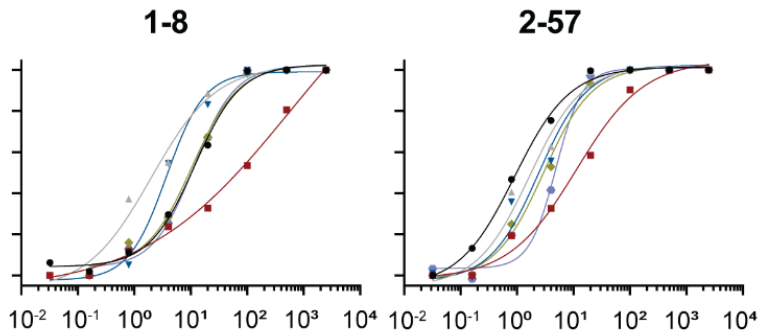

2-43

2-45

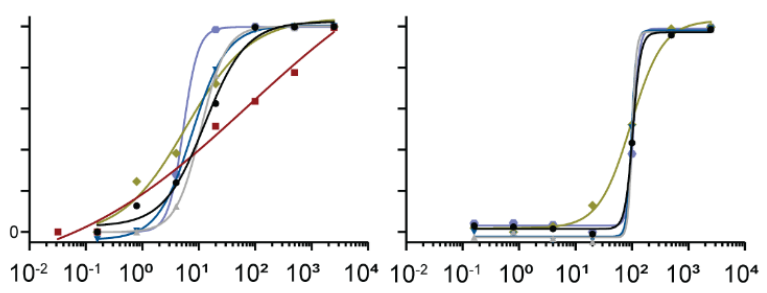

$1-22$

2-28

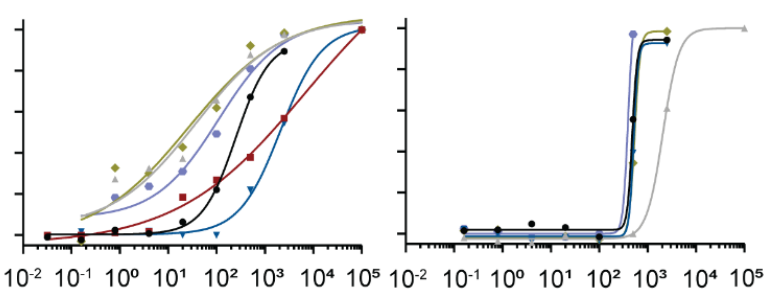

$\mathrm{Nb}$ concentration $(\mathrm{nM})$

$\mathrm{Nb}$ concentration $(\mathrm{nM})$

- SARS-CoV-2 D614G

* SARS-CoV-2 Alpha

- SARS-CoV-2 Beta

- SARS-CoV-2 Lambda

- SARS-CoV-2 Delta

- SARS-CoV

Fig. S5. Neutralization assays of 16 psNbs against pseudotyped SARS-CoV-2 (Wuhan-Hu-1, D614G), the VOCs, VOI and SARS-CoV. 
bioRxiv preprint doi: $\mathrm{https}$ //doi.org/10.1101/2021.12.26.474192; this version posted December 28, 2021. The copyright holder for this preprint (which was not certified by peer review) is the author/funder, who has granted bioRxiv a license to display the preprint in perpetuity. It is made available under aCC-BY-NC-ND 4.0 International license.

\section{Figure S6}

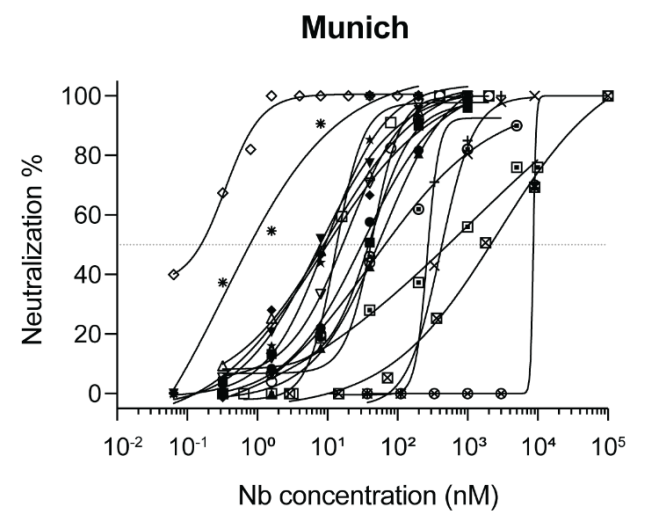

$$
\begin{aligned}
& \text { - 2-32 } \\
& \text { - } 2-34 \\
& +2-38 \\
& +2-31 \\
& \text { - 1-8 } \\
& -2-43 \\
& \rightarrow 1-20 \\
& \triangle \quad 2-30 \\
& \rightarrow 2-35 \\
& -2-67
\end{aligned}
$$

Delta

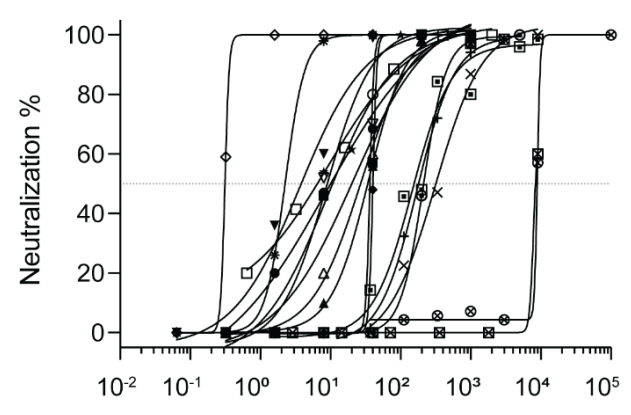

$\mathrm{Nb}$ concentration $(\mathrm{nM})$

Fig. S6. PRNT assay of 17 presentative psNbs against SARS-CoV-2 (Munich strain) and Delta variant. 
bioRxiv preprint doi: https://doi.org/10.1101/2021.12.26.474192; this version posted December 28, 2021. The copyright holder for this preprint (which was not certified by peer review) is the author/funder, who has granted bioRxiv a license to display the preprint in perpetuity. It is made available under aCC-BY-NC-ND 4.0 International license.

\section{Figure S7}

A

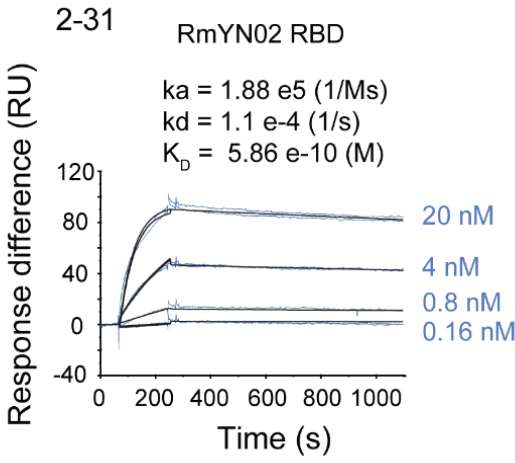

C

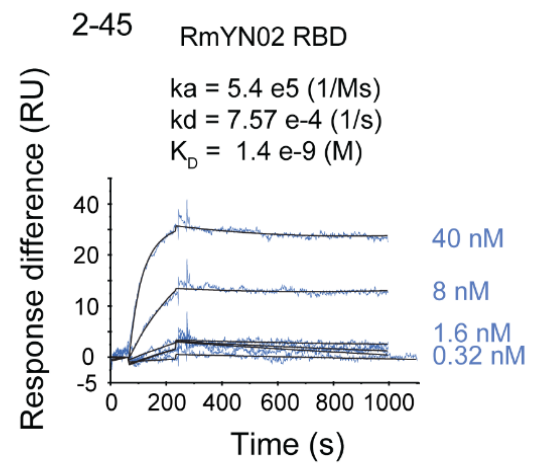

E

$2-45$

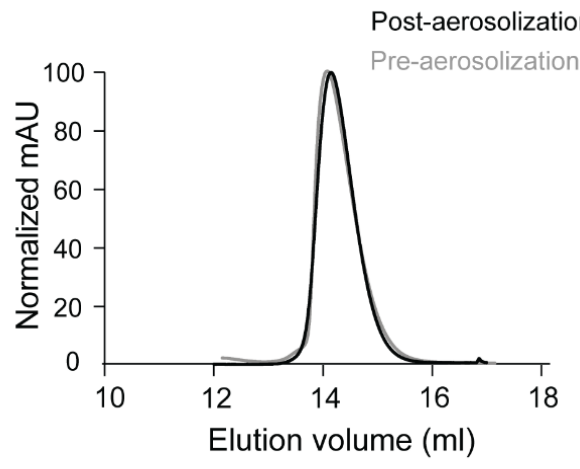

B

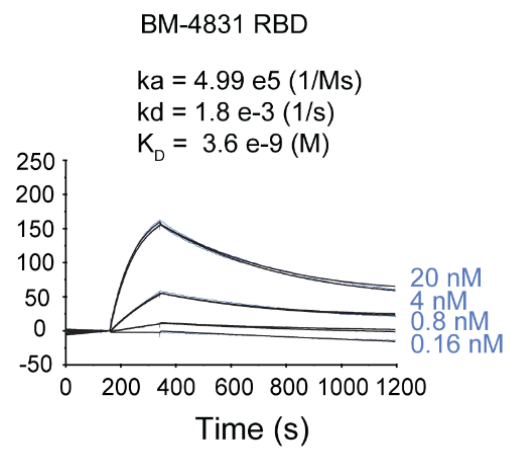

D

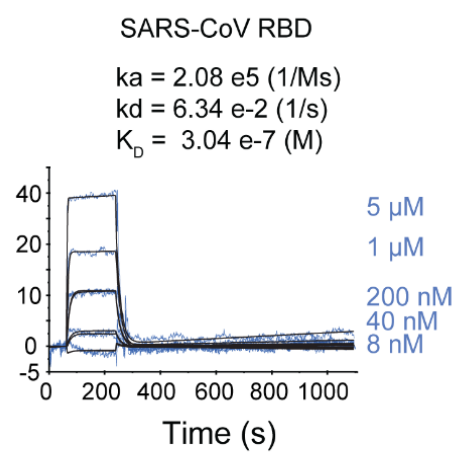

$\mathbf{F}$ Pseudovirus neutralization SARS-CoV-2 (Wuhan-Hu-1, D614G)

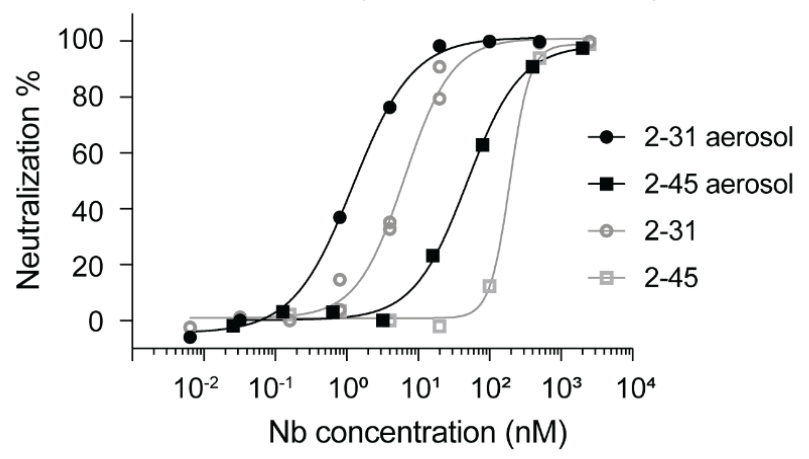

Fig. S7. Biophysical characterizations of highly broad psNbs (2-31 and 2-45). (A-B) SPR kinetic measurement of 2-31 against $\mathrm{RBD}_{\mathrm{RmYN02}}$ and RBD вм-4831. (C-D) SPR kinetic measurement of 2-45 against $\mathrm{RBD}_{\text {SARS-Cov }} \mathrm{RBD}_{\text {BM-4831. }}$ (E) SEC analysis of 2-45 pre and post aerosolization. (F) Pseudovirus neutralization of 2-31 and 2-45 pre and post aerosolization. 


\section{Figure S8}

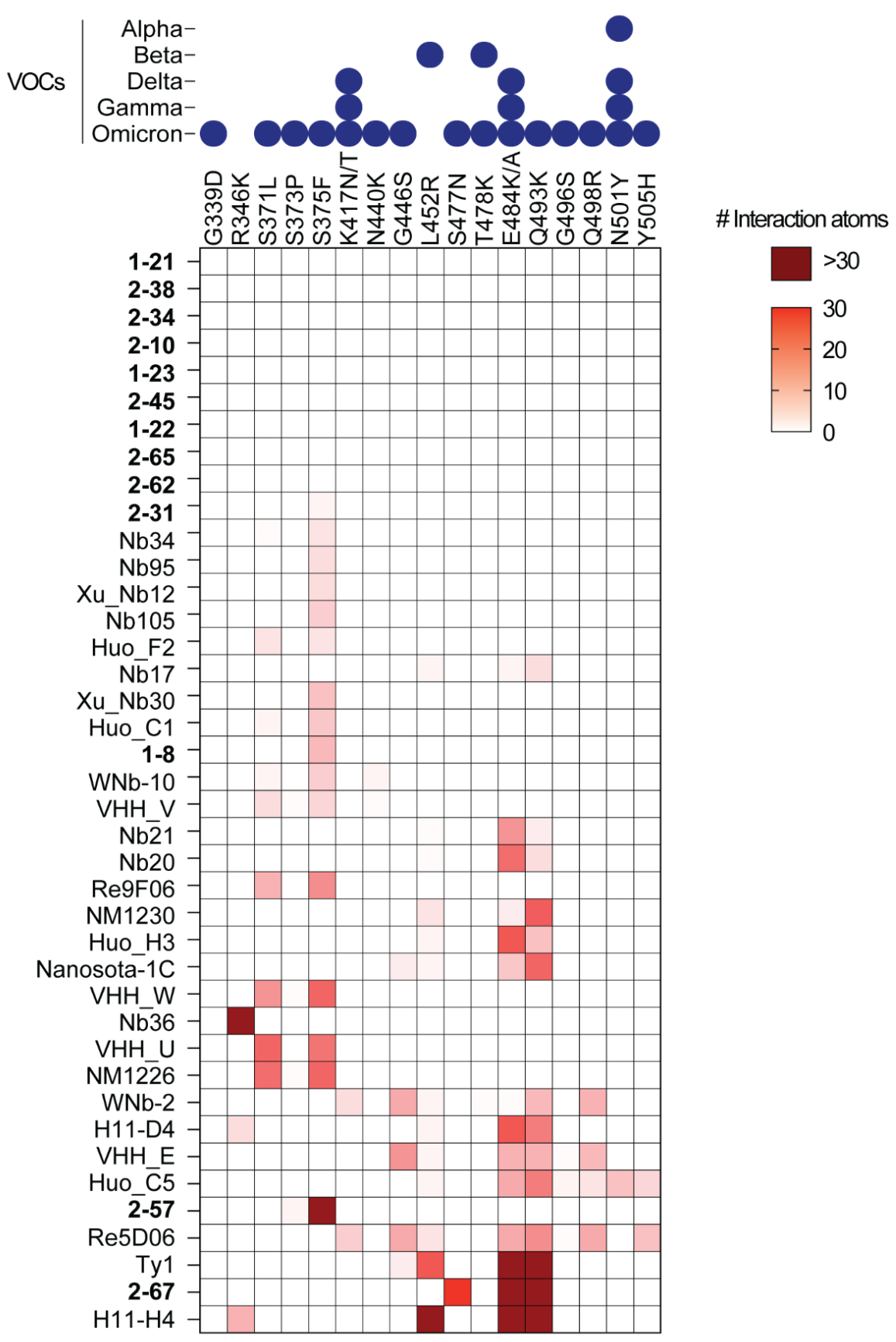

Fig. S8. Structural analysis of Nb binding to the RBD mutations from SARS-CoV-2 VOCs.

Interfaces were extracted from PDB Nb structures. The number of Nb-interacting atoms per each VOC residue was counted to generate the plot. The psNbs identified in this study are bolded. 


\section{Figure S9}

A

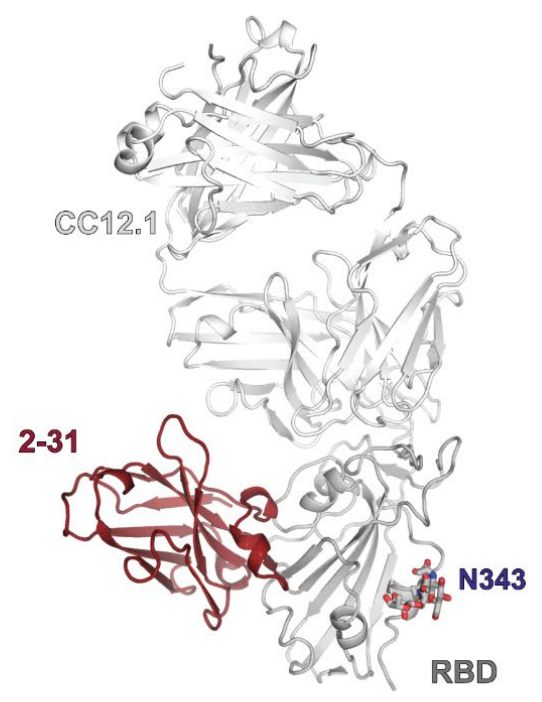

C

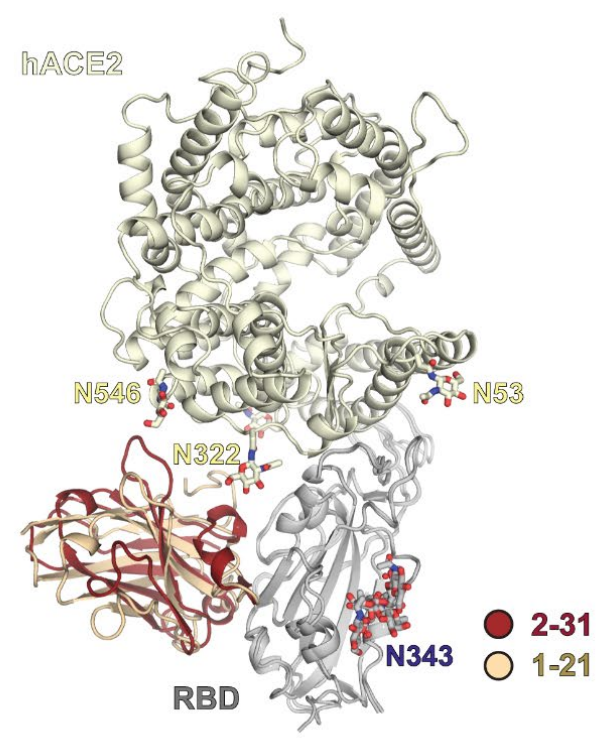

B

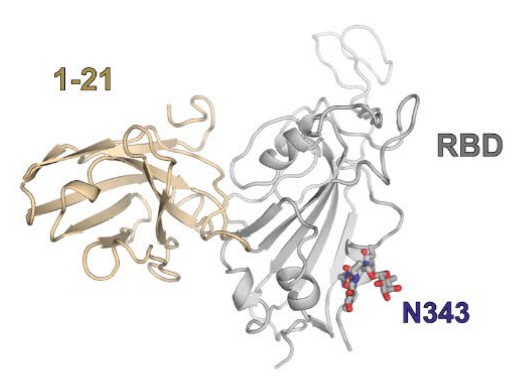

Fig. S9. Comparison of overall structures of 2-31 and 1-21 bound to SARS-CoV-2 RBD.

Structures are shown as ribbon representation. Glycans in SARS-CoV-2 RBD and ACE2 are shown in sticks and labeled with their corresponding asparagine $(\mathrm{N})$ position number. CC12.1 was used to complex with 2-31 and SARS-CoV-2 to obtain diffraction-quality crystals for structure determination. 2-31 is shown in dark red, 121 in beige, RBD in light gray, ACE2 in light yellow, and CC12.1 in white. Structures of 2-31, 1-21, and ACE2 bound to SARS-CoV-2 are superimposed for comparison in $\mathbf{c}$. 


\section{Figure S10}

\section{A}

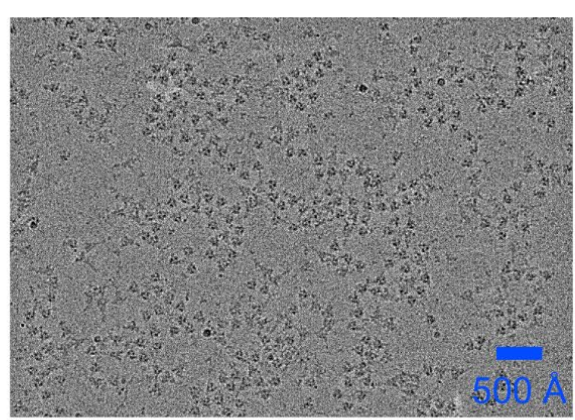

B

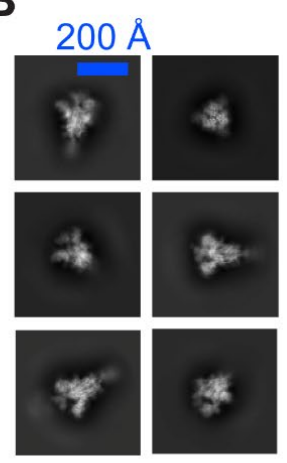

C

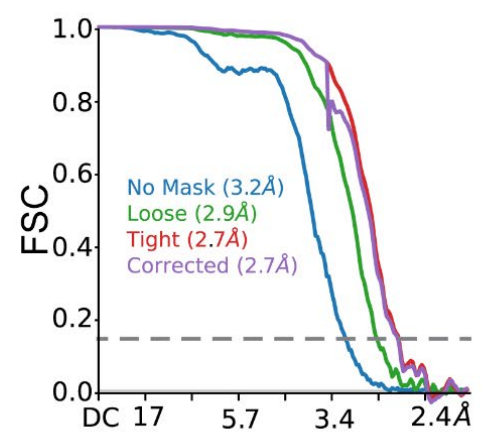

D

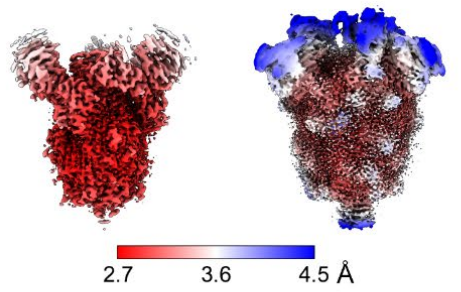

E mask $\mathbf{F}$

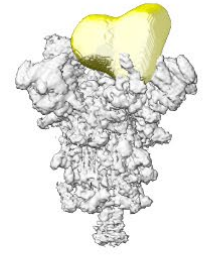

F focused 3D classification

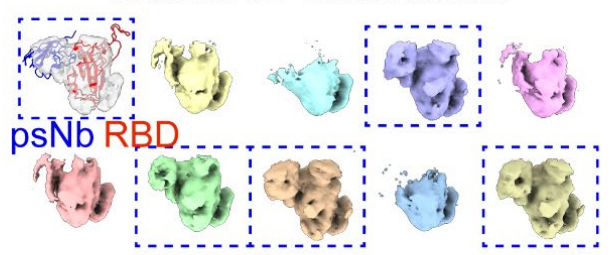

Fig. S10. Cryo-EM Structure determination workflow illustrated by 2-57. (A) Representative micrograph of spike with 2-57. (B) Representative 2D class averages of the spike glycoprotein with 2-57. (C) Gold-standard Fourier shell correlation (FSC) curves calculated from two independently refined half-maps with different masks for spike with 2-57. (D) Local resolution map shown at two density thresholds (left: 0.5 and right: 0.1 ). (E) Masking of the RBD:psNb density for focused classification. (F) Results of focused classification. Classes with strong RBD:psNb densities (blue rectangle boxes) are selected for further local refinements. 


\section{Figure S11}

\section{A}

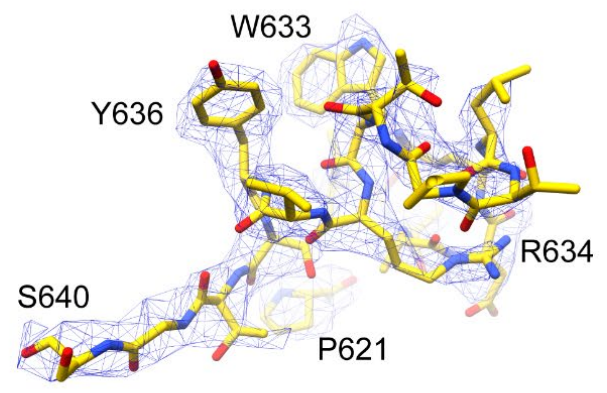

B
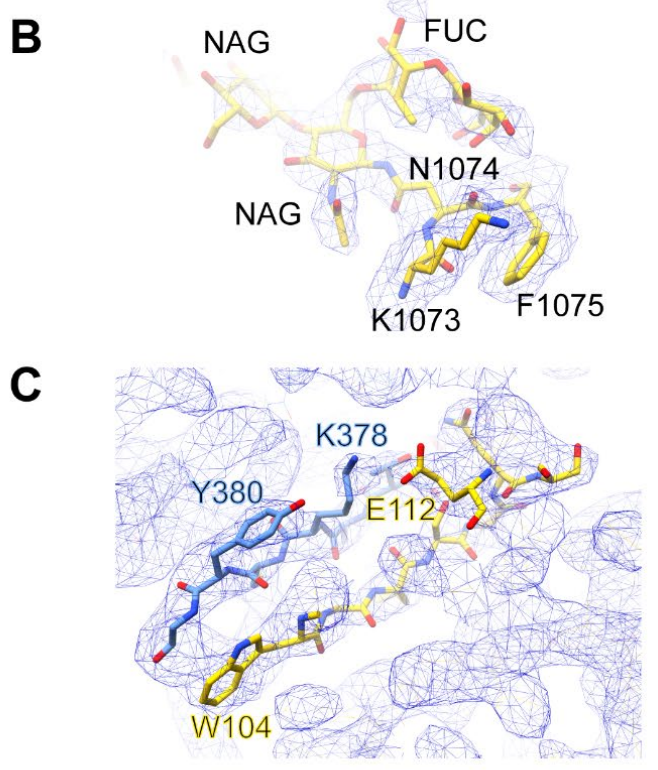

Fig. S11. Representative cryo-EM densities of psNbs in complex with the spike or RBD. (A) Highresolution cryo-EM maps allow the modeling of residue 621 to 640 for the spike protein, which are absent in the starting structure (PDB: 7CAK). The map was contoured at level 0.15. (B) High-resolution cryoEM maps enable the delineation of complex glycan composition at Asn1074 with a Fucose (FUC) attached to the first Nacetylglucosamine (NAG). The map was contoured at level 0.15. (C) High-resolution cryoEM maps facilitated modeling of the RBD (blue): 2-57 (yellow) interface. The map was contoured at level 0.64. 


\section{Figure S12}

A

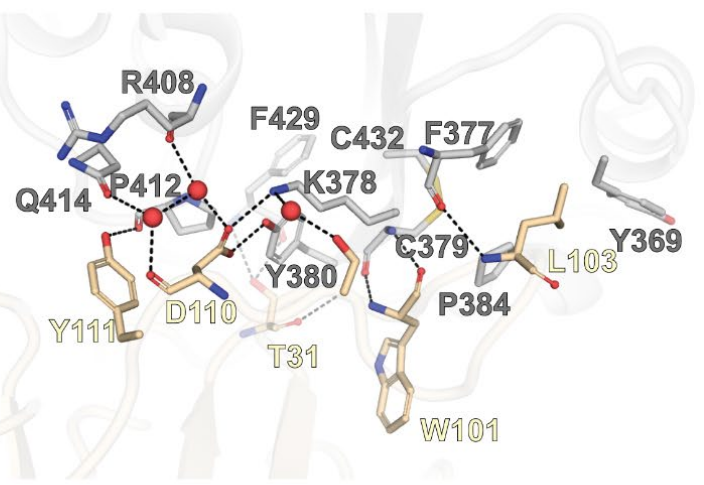

C

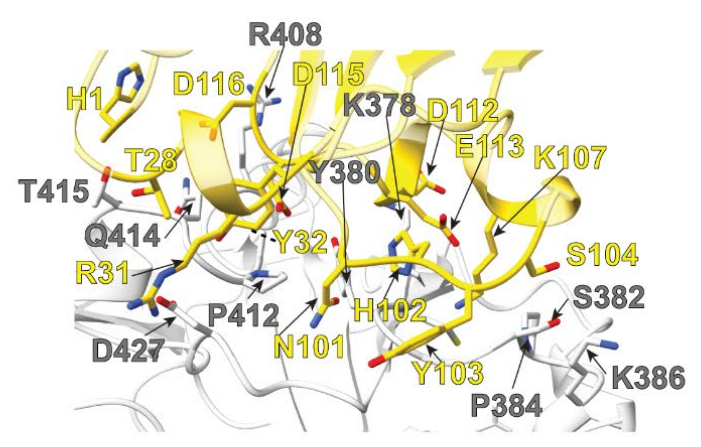

$\mathbf{E}$

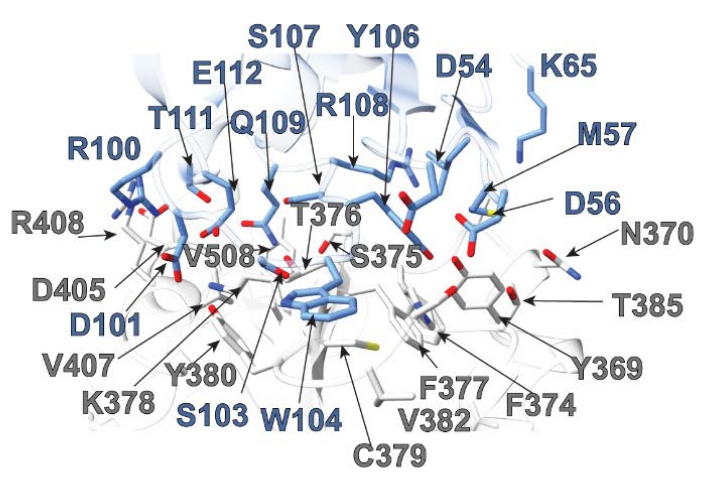

E
B

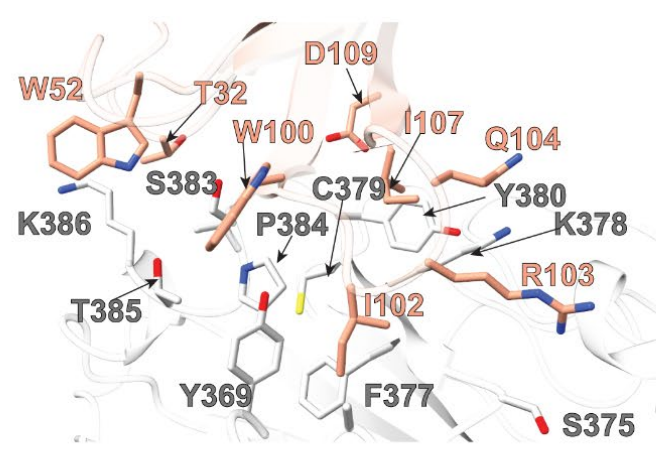

D

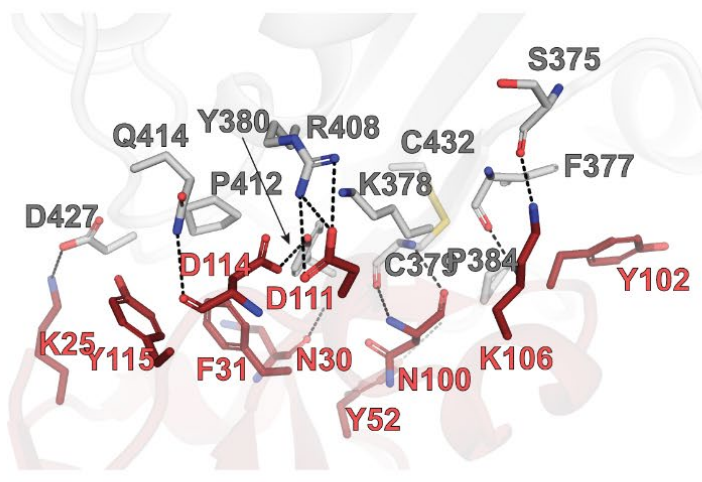

Fig. S12. Interactions of epitope II psNbs in complex with the spike or RBD. Key residues involved in the RBD: psNb interfaces are shown in sticks. Hydrogen bonds or salt bridges are indicated with dashed lines. Water molecules mediating interaction between 1-21 and SARS-CoV-2 RBD are shown in red spheres. 1-21 in navajo white, 2-38 in dark salmon, 2-34 in gold, 2-31 in reddish brown, 2-57 in cornflower blue and RBD in light gray. (A) Interactions between RBD and 1-21. (B) Interactions between RBD and 2-38. (C) Interactions between RBD and 2-34. (D) Interactions between RBD and 2-31. (E) Interactions between RBD and 2-57. 


\section{Figure S13}

A

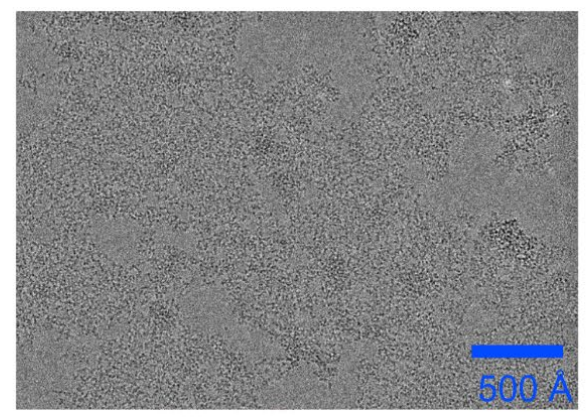

C

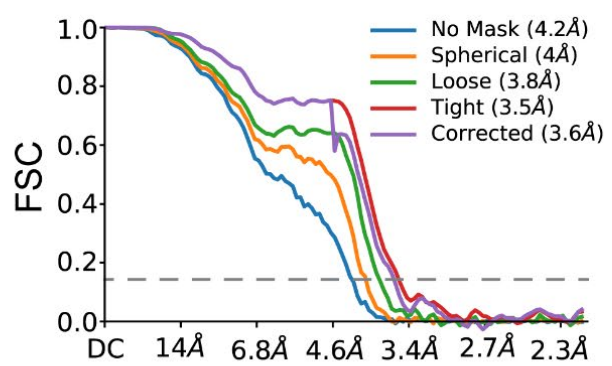

B

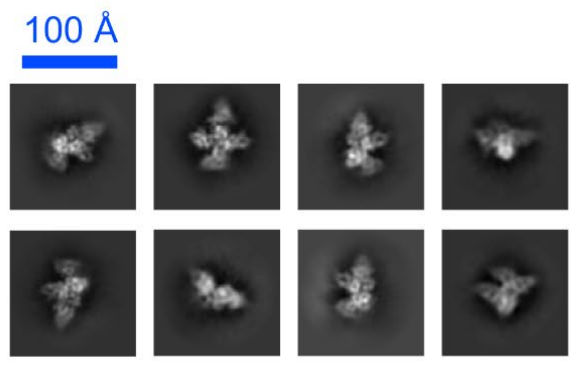

D

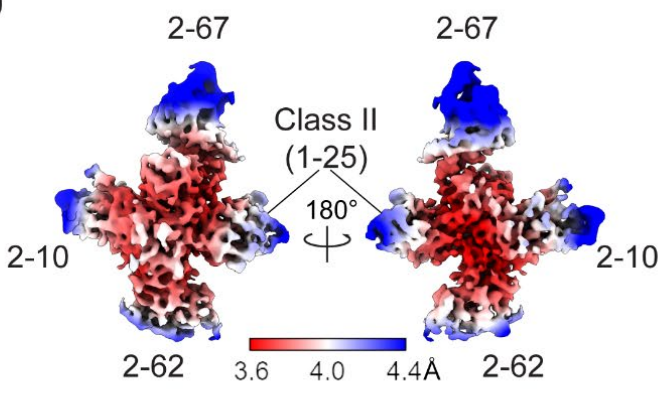

Fig. S13. Cryo-EM Structure determination of RBD with 2-67, 2-10, 2-62 and a class II psNb (1-25) and focused refinement. (A) Representative micrograph of RBD with four psNbs. (B) Representative 2D class averages of RBD and psNbs complex. (C) Gold-standard Fourier shell correlation (FSC) curves calculated from two independently refined half-maps with different masks for RBD and psNbs complex. (D) Local resolution map contoured at level 0.36 . 


\section{A}

\section{Figure S14}

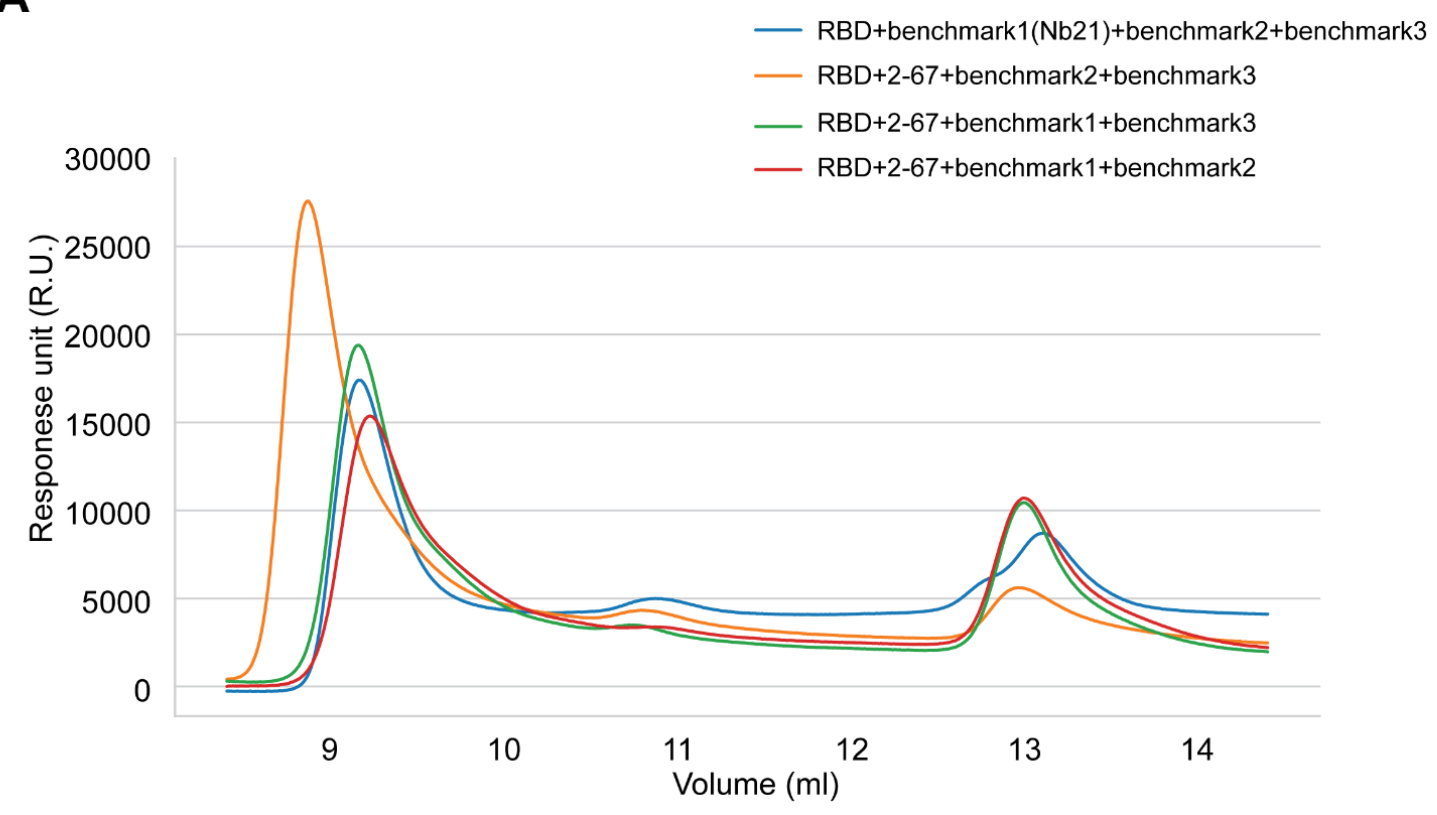

B

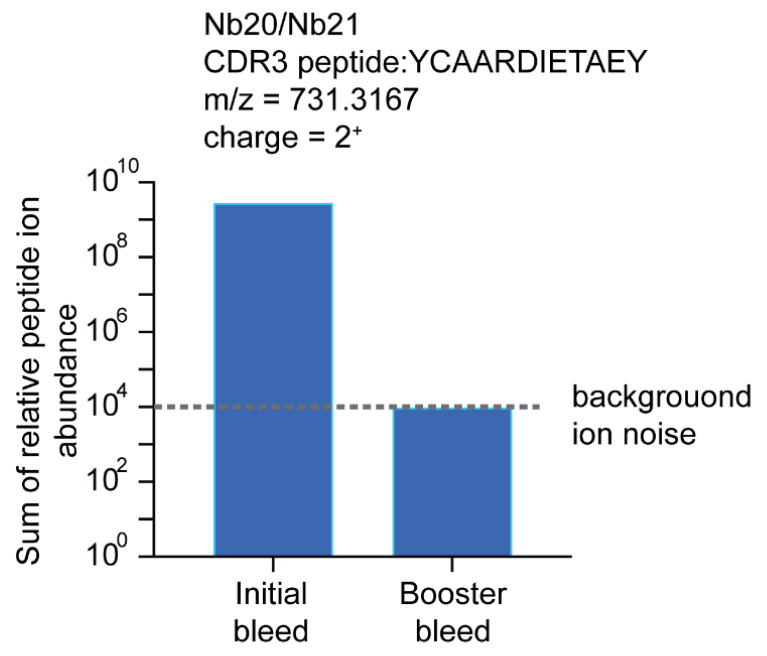

Fig. S14. SEC analysis of 2-67 with RBD and benchmark Nbs, and quantitative MS evaluation of Nbs 20 and 21. (A) Competitive SEC analysis of 2-67 with Nb21 and other high-affinity benchmark Nbs for RBD binding. (B) MS quantification of a CDR3 tryptic peptide specific to Nbs 20 and 21. The $Y$ axis represents the total MS1 ion abundance (relative abundance) of the peptide. Signals below $10^{4-5}$ are generally considered chemical backgrounds. 


\section{Figure S15}

A

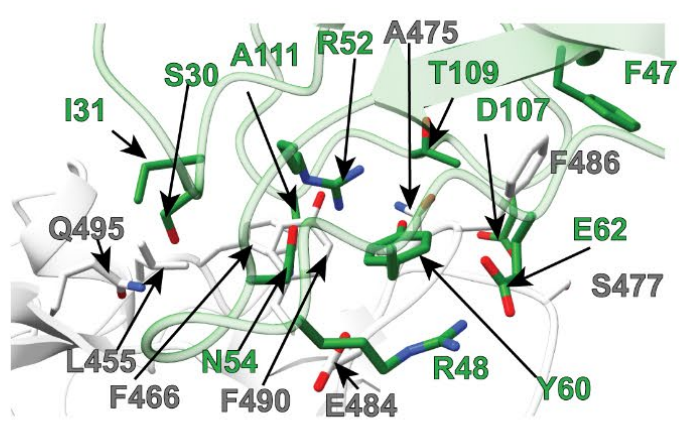

B

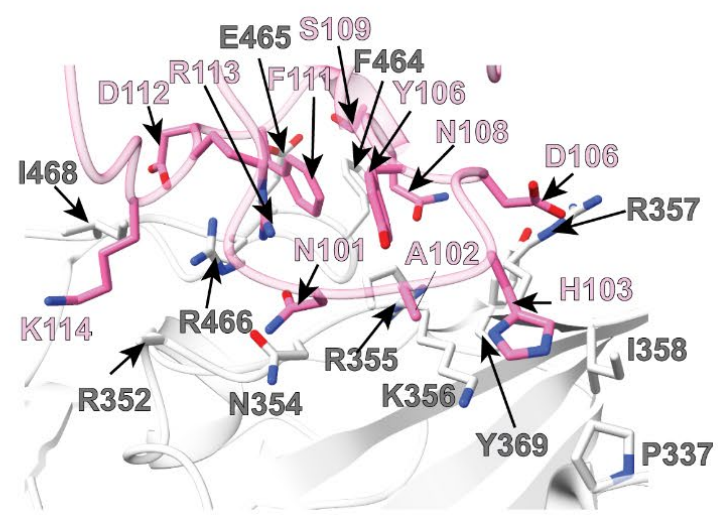

C

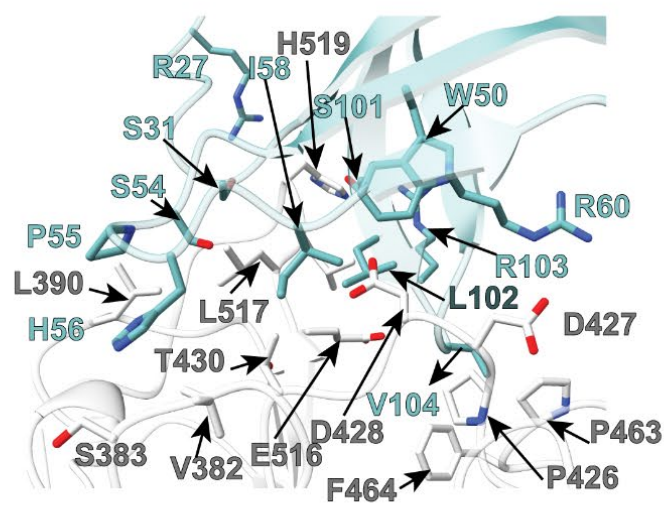

$\mathbf{E}$

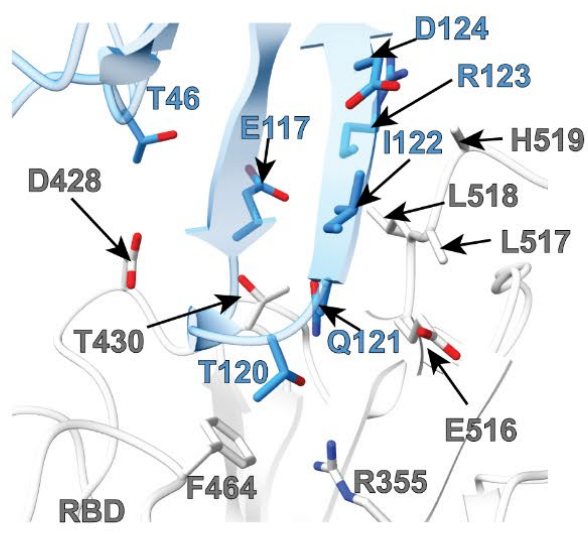

D

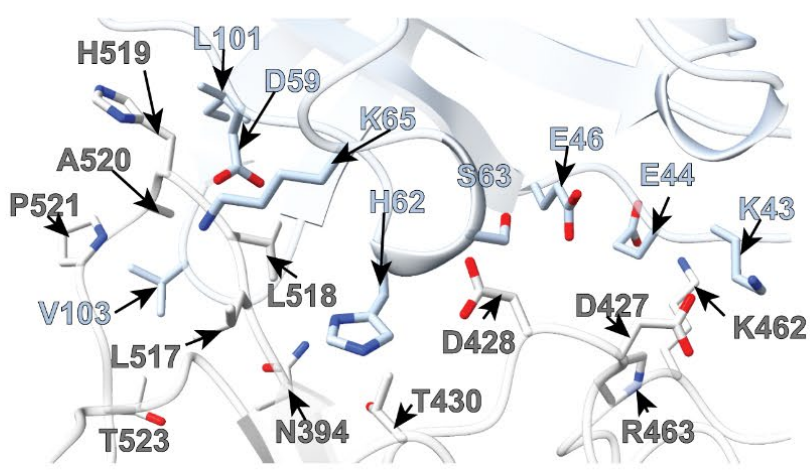

$\mathbf{F}$

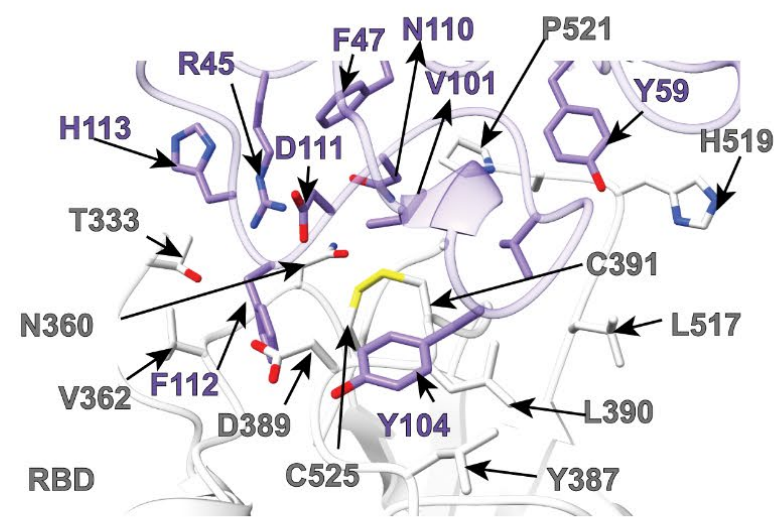

O RBBD

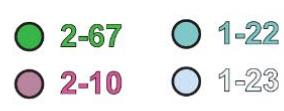

Fig. S15. Interactions of class I, III, IV and V psNbs in complex with SARS-CoV-2 spike or RBD. (A) Interactions between RBD and 2-67. (B) Interactions between RBD and 2-10. (C) Interactions between RBD and 1-22. (D) Interactions between RBD and 1-23. (E) Interactions between RBD and 2-65. (F) Interactions between RBD and 2-62. 
bioRxiv preprint doi: https://doi.org/10.1101/2021.12.26.474192; this version posted December 28, 2021. The copyright holder for this preprint (which was not certified by peer review) is the author/funder, who has granted bioRxiv a license to display the preprint in perpetuity. It is made available under aCC-BY-NC-ND 4.0 International license.

\section{Figure S16}
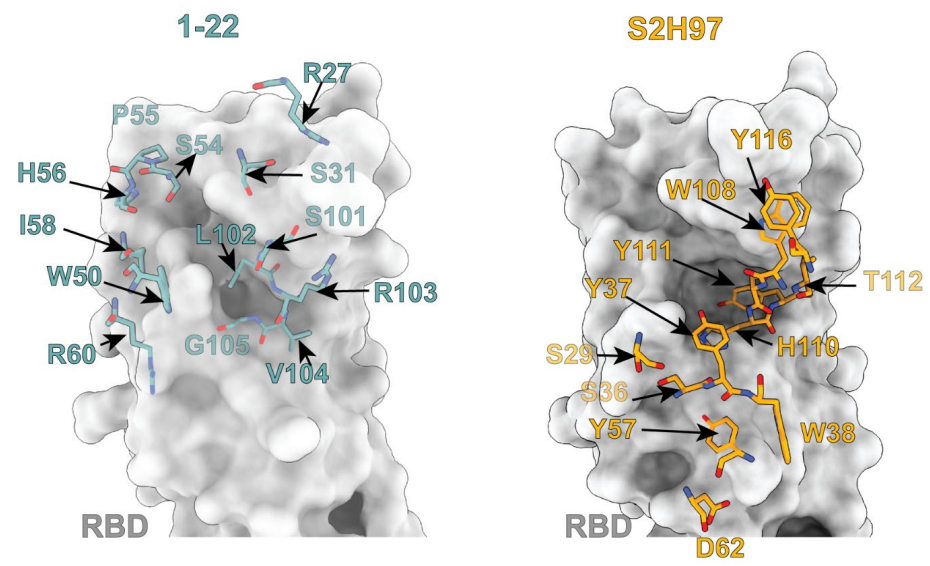

Fig. S16. Structural comparison of 1-22 and an IgG antibody (S2H97) binding to the RBD SARS-CoV-2. 
bioRxiv preprint doi: https://doi.org/10.1101/2021.12.26.474192; this version posted December 28, 2021. The copyright holder for this preprint (which was not certified by peer review) is the author/funder, who has granted bioRxiv a license to display the preprint in perpetuity. It is made available under aCC-BY-NC-ND 4.0 International license.

\section{Figure S17}

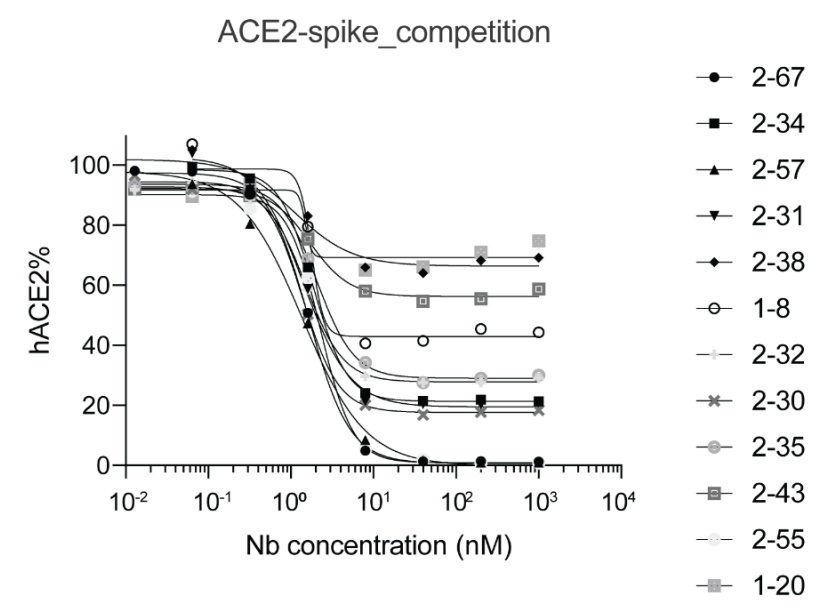

Fig. S17. hACE2 competition assay with SARS-CoV-2 spike by ELISA.

Figure S18

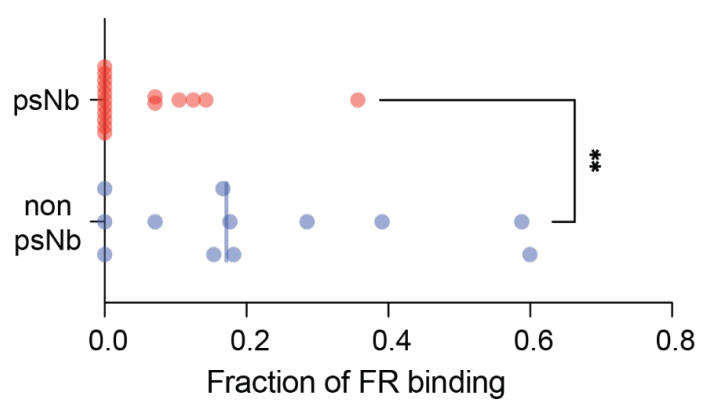

Fig. S18. Comparison of the utility of framework residues of psNbs and non-psNbs for binding to RBD. 
bioRxiv preprint doi: https://doi.org/10.1101/2021.12 26.474192. this version posted December 28, 2021. The copyright holder for this preprint (which was not certified by peer review) is the author/funder, who has granted bioRxiv a license to display the preprint in perpetuity. It is made available under aCC-BY-NC-ND 4.0 International license.

\section{Figure S19}

A

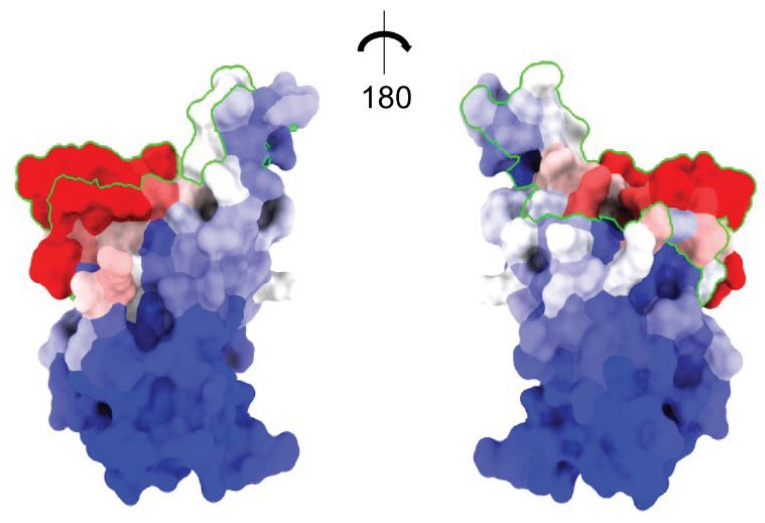

B

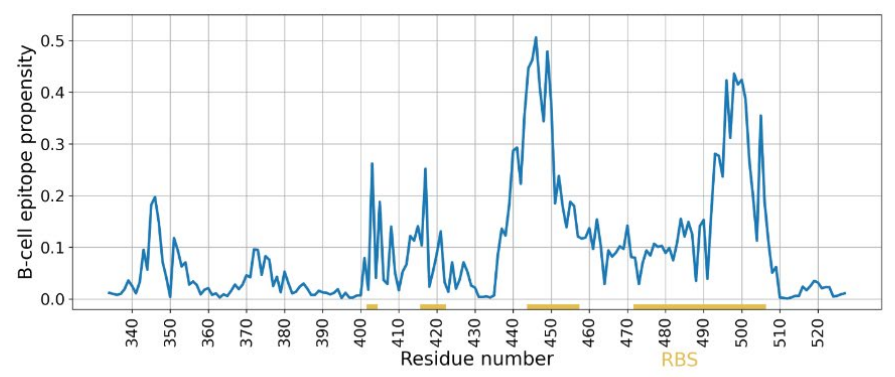

Fig. S19. RBD epitope prediction by ScanNet. (A) The RBD surface is colored by epitope propensity (from blue $=0.0$ to red $=0.35$ and higher) using ChimeraX. The ACE2 binding site is traced by a green line. (B) RBD epitope propensity profile. 
bioRxiv preprint doi: https://doi.org/10.1101/2021.12.26.474192; this version posted December 28, 2021. The copyright holder for this preprint (which was not certified by peer review) is the author/funder, who has granted bioRxiv a license to display the preprint in perpetuity. It is made available under aCC-BY-NC-ND 4.0 International license.

\section{Figure S20}
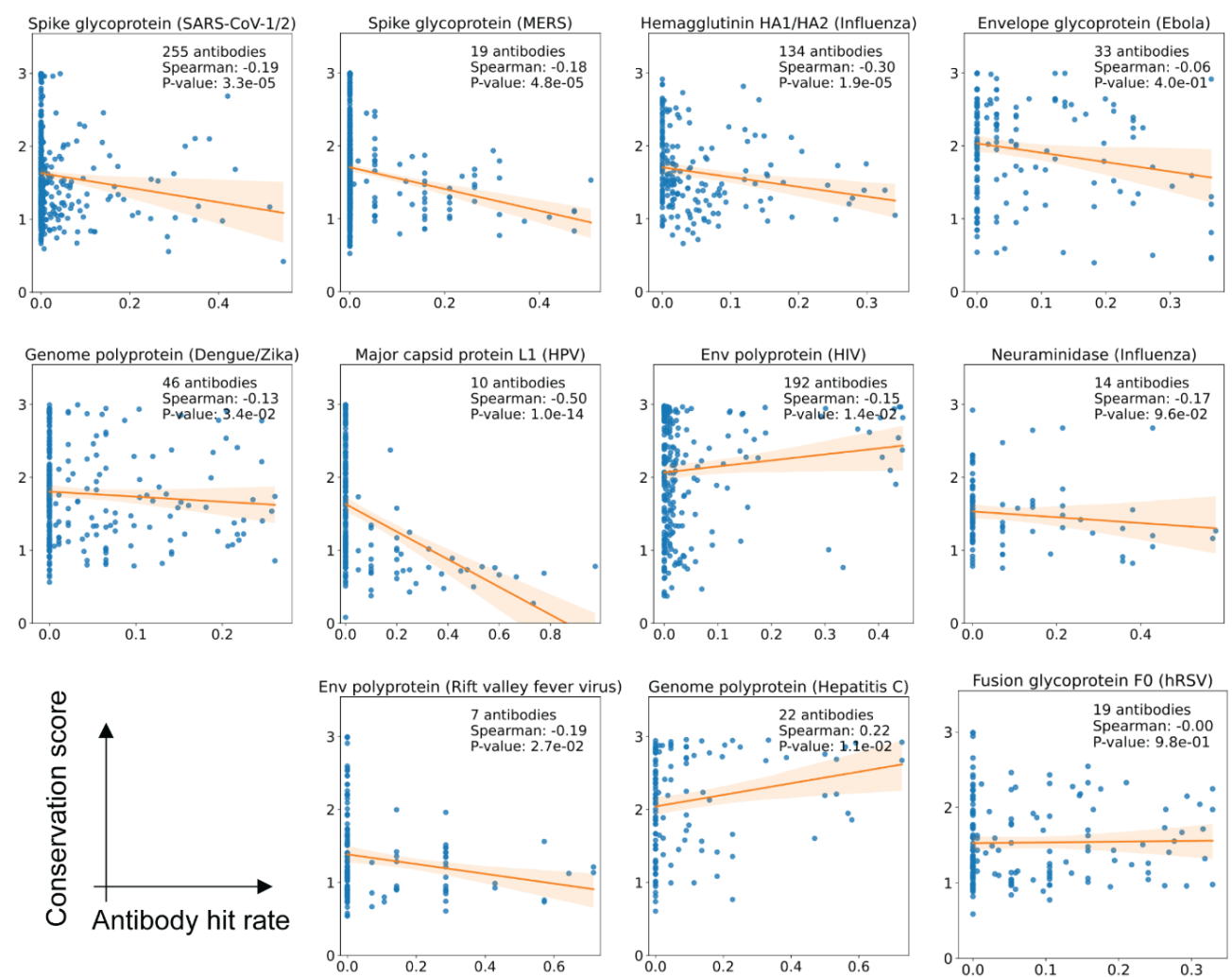

Fig. S20. Scatter plots of antibody hit rates against conservation scores for surface residues for 11 antigens. A negative trend is found for most antigens. Notable outliers include the envelope protein of HIV and the genome polyprotein of Hepatitis C; we speculate that this is due to (i) over-representation of broadly neutralizing antibodies in the PDB and/or (ii) extensive glycosylation. 
bioRxiv preprint doi: https://doi.org/10.1101/2021.12.26.474192; this version posted December 28, 2021. The copyright holder for this preprint (which was not certified by peer review) is the author/funder, who has granted bioRxiv a license to display the preprint in perpetuity. It is made available under aCC-BY-NC-ND 4.0 International license.

\section{Figure S21}
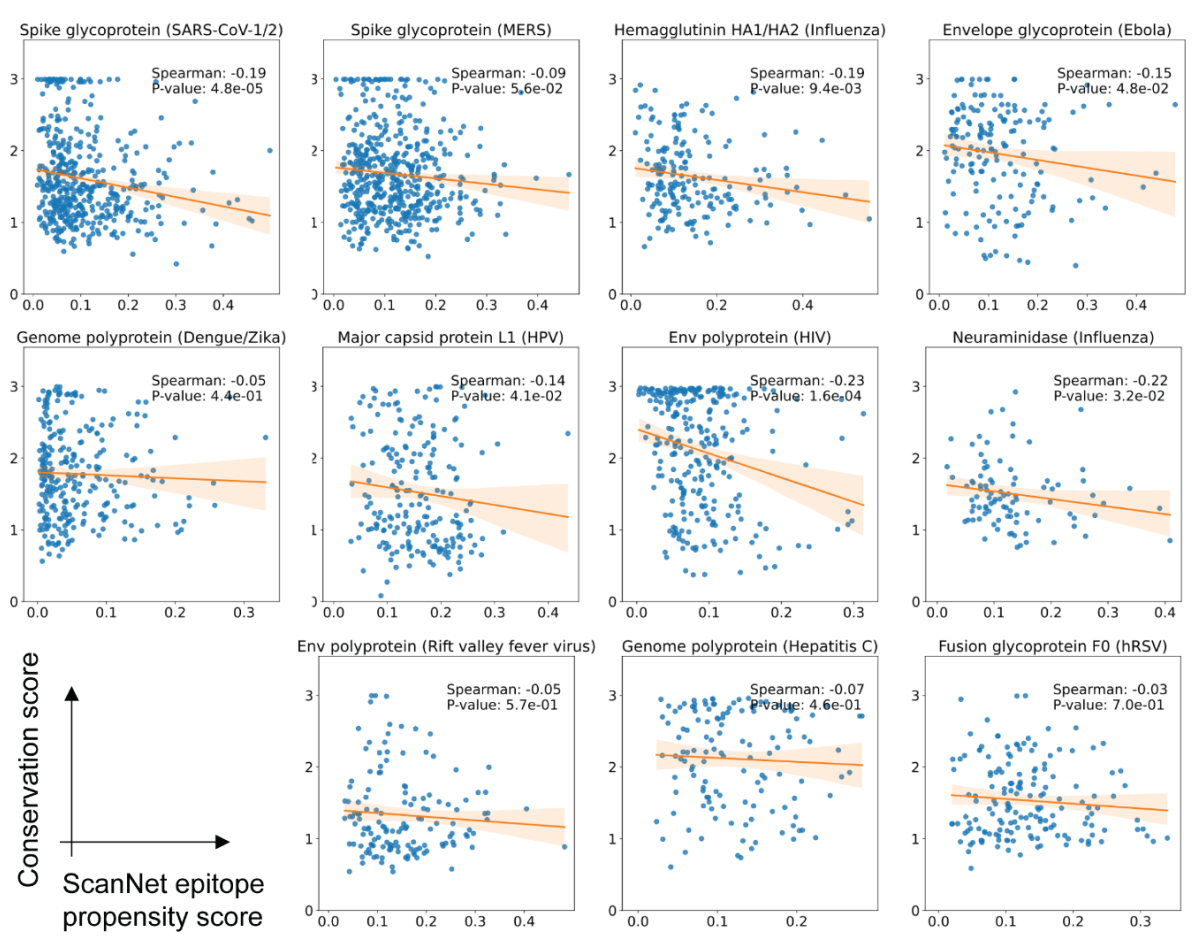

Fig. S21. Scatter plots of ScanNet epitope propensity scores against conservation scores of surface residues for 11 antigens. A negative trend is observed between the conservation score and the ScanNet epitope propensity score which was calculated from antigen structure only. This suggests that the association between conservation and antibody hit rate arises solely from structural properties. 


\section{References}

43. A. A. Cohen et al., Mosaic nanoparticles elicit cross-reactive immune responses to zoonotic coronaviruses in mice. Science 371, 735-741 (2021).

44. C. L. Hsieh et al., Structure-based design of prefusion-stabilized SARS-CoV-2 spikes. Science 369, 1501-1505 (2020).

45. P. C. Fridy et al., A robust pipeline for rapid production of versatile nanobody repertoires. Nat Methods 11, 1253-1260 (2014).

46. D. N. Mastronarde, Automated electron microscope tomography using robust prediction of specimen movements. J Struct Biol 152, 36-51 (2005).

47. A. Punjani, J. L. Rubinstein, D. J. Fleet, M. A. Brubaker, cryoSPARC: algorithms for rapid unsupervised cryo-EM structure determination. Nat Methods 14, 290-296 (2017).

48. A. Punjani, H. Zhang, D. J. Fleet, Non-uniform refinement: adaptive regularization improves singleparticle cryo-EM reconstruction. Nat Methods 17, 1214-1221 (2020).

49. A. Punjani, D. J. Fleet, 3D variability analysis: Resolving continuous flexibility and discrete heterogeneity from single particle cryo-EM. J Struct Biol 213, 107702 (2021).

50. S. H. Scheres, S. Chen, Prevention of overfitting in cryo-EM structure determination. Nat Methods $\mathbf{9}$, 853-854 (2012).

51. Z. Lv et al., Structural basis for neutralization of SARS-CoV-2 and SARS-CoV by a potent therapeutic antibody. Science 369, 1505-1509 (2020).

52. P. Emsley, B. Lohkamp, W. G. Scott, K. Cowtan, Features and development of Coot. Acta Crystallogr D Biol Crystallogr 66, 486-501 (2010).

53. M. Mirdita et al., ColabFold - Making protein folding accessible to all. bioRxiv, 2021.2008.2015.456425 (2021).

54. P. V. Afonine et al., Real-space refinement in PHENIX for cryo-EM and crystallography. Acta Crystallogr D Struct Biol 74, 531-544 (2018).

55. P. Emsley, M. Crispin, Structural analysis of glycoproteins: building N-linked glycans with Coot. Acta Crystallogr D Struct Biol 74, 256-263 (2018).

56. E. F. Pettersen et al., UCSF ChimeraX: Structure visualization for researchers, educators, and developers. Protein Sci 30, 70-82 (2021).

57. J. Zivanov et al., New tools for automated high-resolution cryo-EM structure determination in RELION3. Elife 7, (2018).

58. D. A. Case et al., The Amber biomolecular simulation programs. J Comput Chem 26, 1668-1688 (2005).

59. J. C. Phillips et al., Scalable molecular dynamics with NAMD. J Comput Chem 26, 1781-1802 (2005).

60. C. Tian et al., ff19SB: Amino-Acid-Specific Protein Backbone Parameters Trained against Quantum Mechanics Energy Surfaces in Solution. J Chem Theory Comput 16, 528-552 (2020).

61. K. N. Kirschner et al., GLYCAM06: a generalizable biomolecular force field. Carbohydrates. J Comput Chem 29, 622-655 (2008).

62. W. L. Jorgensen, J. Chandrasekhar, J. D. Madura, R. W. Impey, M. L. Klein, Comparison of simple potential functions for simulating liquid water. J Chem Phys 79, 926-935 (1983).

63. G. J. Martyna, D. J. Tobias, M. L. Klein, Constant pressure molecular dynamics algorithms. J Chem Phys 101, 4177-4189 (1994).

64. S. E. Feller, Y. Zhang, R. W. Pastor, B. R. Brooks, Constant pressure molecular dynamics simulation: The Langevin piston method. J Cheml Phys 103, 4613-4621 (1995).

65. G. C. Jean-Paul Ryckaert, Herman J.C Berendsen, Numerical integration of the cartesian equations of motion of a system with constraints: molecular dynamics of n-alkanes. J Comput Phys 23, 327-341 (1977).

66. H. Gohlke, D. A. Case, Converging free energy estimates: MM-PB(GB)SA studies on the proteinprotein complex Ras-Raf. J Comput Chem 25, 238-250 (2004).

67. P. A. Kollman et al., Calculating structures and free energies of complex molecules: combining molecular mechanics and continuum models. Acc Chem Res 33, 889-897 (2000).

68. B. R. Miller, 3rd et al., MMPBSA.py: An Efficient Program for End-State Free Energy Calculations. $J$ Chem Theory Comput 8, 3314-3321 (2012).

69. D. C. Ekiert et al., A highly conserved neutralizing epitope on group 2 influenza A viruses. Science 333 , 843-850 (2011). 
70. M. Yuan et al., A highly conserved cryptic epitope in the receptor binding domains of SARS-CoV-2 and SARS-CoV. Science 368, 630-633 (2020).

71. Z. Otwinowski, W. Minor, Processing of X-ray diffraction data collected in oscillation mode. Methods Enzymol 276, 307-326 (1997).

72. A. J. McCoy et al., Phaser crystallographic software. J Appl Crystallogr 40, 658-674 (2007).

73. H. Liu et al., Cross-Neutralization of a SARS-CoV-2 Antibody to a Functionally Conserved Site Is Mediated by Avidity. Immunity 53, 1272-1280 e1275 (2020).

74. C. K. Emsley P, in Acta Crystallogr D Biol Crystallogr. (2004), vol. 12, pp. 2126-2132.

75. P. D. Adams et al., PHENIX: a comprehensive Python-based system for macromolecular structure solution. Acta Crystallogr D Biol Crystallogr 66, 213-221 (2010).

76. J. Tubiana, D. Schneidman-Duhovny, H. J. Wolfson, ScanNet: An interpretable geometric deep learning model for structure-based protein binding site prediction. bioRxiv, 2021.2009.2005.459013 (2021).

77. J. Dunbar et al., SAbDab: the structural antibody database. Nucleic Acids Res 42, D1140-1146 (2014).

78. L. Fu, B. Niu, Z. Zhu, S. Wu, W. Li, CD-HIT: accelerated for clustering the next-generation sequencing data. Bioinformatics 28, 3150-3152 (2012).

79. T. Nakamura, K. D. Yamada, K. Tomii, K. Katoh, Parallelization of MAFFT for large-scale multiple sequence alignments. Bioinformatics 34, 2490-2492 (2018).

80. M. Steinegger et al., HH-suite3 for fast remote homology detection and deep protein annotation. BMC Bioinformatics 20, 473 (2019).

81. H. Ashkenazy et al., ConSurf 2016: an improved methodology to estimate and visualize evolutionary conservation in macromolecules. Nucleic Acids Res 44, W344-350 (2016).

82. J. Huang et al., CHARMM36m: an improved force field for folded and intrinsically disordered proteins. Nat Methods 14, 71-73 (2017).

83. R. C. Edgar, MUSCLE: a multiple sequence alignment method with reduced time and space complexity. BMC Bioinformatics 5, 113 (2004).

84. S. Kumar, G. Stecher, M. Li, C. Knyaz, K. Tamura, MEGA X: Molecular Evolutionary Genetics Analysis across Computing Platforms. Mol Biol Evol 35, 1547-1549 (2018).

85. S. Mitternacht, FreeSASA: An open source C library for solvent accessible surface area calculations. F1000Res 5, 189 (2016).

86. S. Jo, T. Kim, V. G. Iyer, W. Im, CHARMM-GUI: a web-based graphical user interface for CHARMM. J Comput Chem 29, 1859-1865 (2008).

87. C. J. Williams et al., MolProbity: More and better reference data for improved all-atom structure validation. Protein Sci 27, 293-315 (2018). 